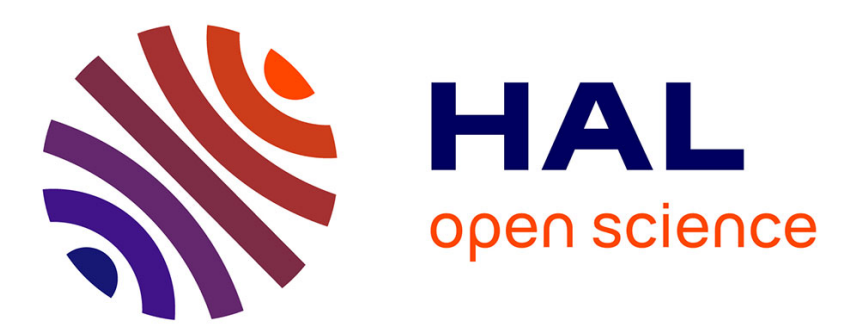

\title{
Convergence of the Lagrange-Galerkin method for the Equations Modelling the Motion of a Fluid-Rigid System
}

Jorge San Martin, Jean-François Scheid, Takéo Takahashi, Marius Tucsnak

\section{To cite this version:}

Jorge San Martin, Jean-François Scheid, Takéo Takahashi, Marius Tucsnak. Convergence of the Lagrange-Galerkin method for the Equations Modelling the Motion of a Fluid-Rigid System. SIAM Journal on Numerical Analysis, 2005, Volume 43, Issue 4, pp.1536-1571. 10.1137/S0036142903438161. hal-00092094

\section{HAL Id: hal-00092094 https://hal.science/hal-00092094}

Submitted on 8 Sep 2006

HAL is a multi-disciplinary open access archive for the deposit and dissemination of scientific research documents, whether they are published or not. The documents may come from teaching and research institutions in France or abroad, or from public or private research centers.
L'archive ouverte pluridisciplinaire HAL, est destinée au dépôt et à la diffusion de documents scientifiques de niveau recherche, publiés ou non, émanant des établissements d'enseignement et de recherche français ou étrangers, des laboratoires publics ou privés. 


\title{
Convergence of the Lagrange-Galerkin method for the Equations Modelling the Motion of a Fluid-Rigid System
}

\author{
JoRGe SAN MARTÍN, \\ Departemento de Ingeniería Matemática, Universad de Chile, \\ Casilla 170/3-Correo 3, Santiago, Chile. \\ (jorge@dim.uchile.cl). \\ JeAn-François Scheid, TAKÉO TAKAhashi, Marius TuCsnak \\ Institut Elie Cartan, Faculté des Sciences, BP239, \\ 54506 Vandoeuvre-lès-Nancy, Cedex, France. \\ (Jean-Francois.Scheid@iecn.u-nancy.fr, \\ Takeo.Takahashi@iecn.u-nancy.fr, \\ Marius. Tucsnak@iecn.u-nancy.fr). \\ and \\ INRIA Lorraine, Projet CORIDA.
}

\begin{abstract}
In this paper, we consider a Lagrange-Galerkin scheme to approximate a two dimensional fluid-rigid body problem. The equations of the system are the NavierStokes equations in the fluid part, coupled with ordinary differential equations for the dynamics of the rigid body. In this problem, the equations of the fluid are written in a domain whose variation is one of the unknowns. We introduce a numerical method based on the use of characteristics and on finite elements with a fixed mesh. Our main result asserts the convergence of this scheme.
\end{abstract}

\section{Introduction.}

The aim of this paper is to analyze a Lagrange-Galerkin approximation of the equations modelling the motion of a two-dimensional rigid body immersed in a fluid. We first briefly describe the equations modelling this system. Assume that the system fluid-rigid body occupies a bounded domain $\mathcal{O}$ in $\mathbb{R}^{2}$ with a regular boundary $\partial \mathcal{O}$. The solid is supposed to occupy at each instant $t$ a closed connected subset $B(t) \subset \mathcal{O}$ which is surrounded by 
a viscous homogeneous fluid filling the domain $\Omega(t)=\mathcal{O} \backslash B(t)$.

The motion of the fluid is described by the classical Navier-Stokes equations, whereas the motion of the rigid body is governed by the balance equations for linear and angular momentum (Newton's laws). More precisely, we consider the following system coupling partial differential and ordinary differential equations.

$$
\begin{aligned}
& \rho_{f} \frac{\partial \mathbf{u}}{\partial t}-\nu \Delta \mathbf{u}+\rho_{f}(\mathbf{u} \cdot \nabla) \mathbf{u}+\nabla p=\rho_{f} \mathbf{f}, \quad \mathbf{x} \in \Omega(t), t \in[0, T], \\
& \operatorname{div} \mathbf{u}=0, \quad \mathbf{x} \in \Omega(t), t \in[0, T], \\
& \mathbf{u}=0, \quad \mathbf{x} \in \partial \mathcal{O}, t \in[0, T], \\
& \mathbf{u}=\boldsymbol{\zeta}^{\prime}(t)+\omega(t)(\mathbf{x}-\boldsymbol{\zeta}(t))^{\perp}, \quad \mathbf{x} \in \partial B(t), t \in[0, T], \\
& M \boldsymbol{\zeta}^{\prime \prime}(t)=-\int_{\partial B(t)} \boldsymbol{\sigma} \mathbf{n} \mathrm{d} \Gamma+\rho_{s} \int_{B(t)} \mathbf{f}(\mathbf{x}, t) \mathrm{d} \mathbf{x}, \quad t \in[0, T], \\
& J \omega^{\prime}(t)=-\int_{\partial B(t)}(\mathbf{x}-\boldsymbol{\zeta}(t))^{\perp} \cdot \boldsymbol{\sigma} \mathbf{n} \mathrm{d} \Gamma+\rho_{s} \int_{B(t)}(\mathbf{x}-\boldsymbol{\zeta}(t))^{\perp} \cdot \mathbf{f}(\mathbf{x}, t) \mathrm{d} \mathbf{x}, \quad t \in[0, T], \\
& \mathbf{u}(\mathbf{x}, 0)=\mathbf{u}_{0}(\mathbf{x}), \quad \mathbf{x} \in \Omega(0), \\
& \boldsymbol{\zeta}(0)=\boldsymbol{\zeta}_{0} \in \mathbb{R}^{2}, \boldsymbol{\zeta}^{\prime}(0)=\boldsymbol{\zeta}_{1} \in \mathbb{R}^{2}, \omega(0)=\omega_{0} \in \mathbb{R} \text {. }
\end{aligned}
$$

In the above equations the unknows are $\mathbf{u}(\mathbf{x}, t)$ (the Eulerian velocity field of the fluid), $p(\mathbf{x}, t)$ (the pressure of the fluid), $\boldsymbol{\zeta}(t)$ (the position of the mass center of the rigid body) and $\omega(t)$ (the angular velocity of the rigid body). The domain $B(t)$ is defined by

$$
B(t)=\left\{\mathbf{R}_{-\theta(t)} \mathbf{y}+\boldsymbol{\zeta}(t), \mathbf{y} \in B\right\}
$$

where

$$
\theta(t)=\int_{0}^{t} \omega(s) \mathrm{d} s
$$

$B=B(0)$ and $\mathbf{R}_{\theta}$ is the rotation matrix of angle $\theta$. Moreover we have denoted by $\partial B(t)$ the boundary of the rigid body at instant $t$ and by $\mathbf{n}(\mathbf{x}, t)$ the unit normal to $\partial B(t)$ at the point $\mathbf{x}$ directed to the interior of the rigid body.

The constants $\rho_{f}$ and $\rho_{s}$ are respectively the density of the fluid and of the rigid body. In the sequel, we assume that the densities of the fluid and of the solid are equal, that is

$$
\rho_{f}=\rho_{s}=1
$$

and that the rigid body is a ball in $\mathbb{R}^{2}$. Assumption (1.10) is clearly restrictive but it is important for the forthcoming analysis, so that it is not clear that it can be removed (see remarks 2.1 and 2.4 below). On the contrary, the assumption that the rigid body is a ball is not essential but it avoids some technicalities.

The constants $M$ and $J$ are the mass and the moment of inertia of the rigid body and the positive constant $\nu$ is the viscosity of the fluid. Moreover, $\mathbf{f}(\mathbf{x}, t)$ is the applied force (per unit mass). 
For all $\mathbf{x}=\left(\begin{array}{l}x_{1} \\ x_{2}\end{array}\right)$, we denote by $\mathbf{x}^{\perp}$ the vector $\mathbf{x}^{\perp}=\left(\begin{array}{c}x_{2} \\ -x_{1}\end{array}\right)$. If $\mathbf{x}, \mathbf{y} \in \mathbb{R}^{2}$, then $\mathbf{x} \cdot \mathbf{y}$ stands for the inner product of $\mathbf{x}$ and $\mathbf{y}$ and $|\mathbf{x}|$ stands for the corresponding norm. We have also denoted by $w^{\prime}$ and $w^{\prime \prime}$ the derivatives of a function $w$ depending only on the time $t$.

Finally, the stress tensor (also called the Cauchy stress) is defined by

$$
\boldsymbol{\sigma}(\mathbf{x}, t)=-p(\mathbf{x}, t) \mathbf{I d}+2 \nu \mathbf{D}(\mathbf{u})
$$

where Id is the identity matrix and $\mathbf{D}(\mathbf{u})$ is the tensor field defined by

$$
D(u)_{k, l}=\frac{1}{2}\left(\frac{\partial u_{k}}{\partial x_{l}}+\frac{\partial u_{l}}{\partial x_{k}}\right)
$$

The main difficulties of this problem are:

- the equations of the structure are coupled with those of the fluid,

- the domain of the fluid is variable and it is one of the unknowns of the problem (we thus have a free boundary problem).

The wellposedness of this type of system has been recently studied in a large number of papers (see, for instance, Desjardins-Esteban [6], Gunzburger-Lee-Seregin [17], San Martín-Starovoitov-Tucsnak [25], Grandmont-Maday [16], Takahashi [27] and the references therein).

The literature on the numerical approximation of the solution of (1.1)-(1.8) also contains a large number of recent papers. A part of these papers is based on an Arbitrary Lagrangian Eulerian (ALE) formulation: see, for example, Grandmont-Guimet-Maday [15], Nobile [22], Maury-Glowinski [21], Maury [19], Maury [20], Formaggia-Nobile [9], Farhat-Geuzaine-Grandmont [8]. In the ALE method, at each time step, the mesh is moved with an arbitrary velocity in the fluid in order to follow the motion of the rigid body. The stability of the ALE method is studied in [9] (in the case of the finite element context) and by [8] (in the case of the finite volume context). We also mention the work of Gastaldi [11] where, in the case of an advection-diffusion equation in a moving twodimensional domain, a priori error estimates which are optimal both in space and time have been obtained.

Another approach, developed by Glowinski et al.([14], [13]) is based on a fictitious domain formulation: the rigid bodies are filled by the surrounding fluid and the constraint of rigid body motion is relaxed by introducing a distributed Lagrange multiplier.

As far as we know, the only proof of the convergence of one of these methods is given in [15] for a simplified problem in one space dimension. The main novelty brought in by our paper consists in the fact that we construct a new approximation method using a fixed mesh and that we prove a convergence result. This method is inspired by the Galerkin-Lagrange approximation which is commonly used for Navier-Stokes equations. (see Pironneau [23] and Süli [26]). 
The remaining part of this paper is organized as follows. In Section 2 we introduce some function spaces and we semi-discretize our problem with respect to the time variable. In Section 3 we give the full discretization of the problem and we state the main result. Section 4 is devoted to the study of the finite element spaces which have been introduced in the previous section. Section 5 is devoted to the study of a change of variables which plays an important role in the proof of the main result. In Section 6 we proof the consistency of our scheme. Finally, in Section 7 we give the proof of the main result.

\section{Notations and preliminaries.}

\subsection{Notations and function spaces.}

Throughout this paper, we shall use the classical Sobolev spaces $H^{s}(\Omega), H_{0}^{s}(\Omega), H^{-s}(\Omega)$, $s \geqslant 0$ and the space of Lipschitz continuous functions $C^{0,1}(\bar{\Omega})$ on the closure of $\Omega$. We also define

$$
L_{0}^{2}(\Omega)=\left\{f \in L^{2}(\Omega) \mid \int_{\Omega} f \mathrm{~d} \mathbf{x}=0\right\}
$$

and denote by $\mathcal{L}_{0}^{2}(\Omega), \mathcal{H}^{s}(\Omega), \mathcal{H}_{0}^{s}(\Omega), \mathcal{H}^{-s}(\Omega), s \geqslant 0$ the spaces $\left[L_{0}^{2}(\Omega)\right]^{2}$, $\left[H^{s}(\Omega)\right]^{2}$, $\left[H_{0}^{s}(\Omega)\right]^{2},\left[H^{-s}(\Omega)\right]^{2}$. The usual inner product in $\mathcal{L}^{2}(\mathcal{O})$ will be denoted by

$$
(\mathbf{u}, \mathbf{v})=\int_{\mathcal{O}} \mathbf{u} \cdot \mathbf{v} \mathrm{d} \mathbf{x} \quad \forall \mathbf{u}, \mathbf{v} \in \mathcal{L}^{2}(\mathcal{O})
$$

If $\mathbf{A}$ is a matrix, we denote by $\mathbf{A}^{*}$ its transpose. For any $2 \times 2$ matrices $\mathbf{A}, \mathbf{B} \in \mathcal{M}_{2 \times 2}$, we denote by $\mathbf{A}: \mathbf{B}$ their inner product $\mathbf{A}: \mathbf{B}=\operatorname{Trace}\left(\mathbf{A}^{*} \mathbf{B}\right)$, and by $|\mathbf{A}|$ the corresponding norm. For convenience, we use the same notation as in (2.1) for the inner product in $L^{2}\left(\mathcal{O}, \mathcal{M}_{2 \times 2}\right)$, that is

$$
(\mathbf{A}, \mathbf{B})=\int_{\mathcal{O}} \mathbf{A}: \mathbf{B} \mathrm{d} \mathbf{x} \quad \forall \mathbf{A}, \mathbf{B} \in L^{2}\left(\mathcal{O}, \mathcal{M}_{2 \times 2}\right)
$$

We also define the spaces

$$
\mathcal{K}(\boldsymbol{\zeta})=\left\{\mathbf{u} \in \mathcal{H}_{0}^{1}(\mathcal{O}) \mid \mathbf{D}(\mathbf{u})=0 \quad \text { in } \quad B(\boldsymbol{\zeta})\right\}
$$

and

$$
\widehat{\mathcal{K}}(\boldsymbol{\zeta})=\left\{\mathbf{u} \in \mathcal{H}_{0}^{1}(\mathcal{O}) \mid \operatorname{div} \mathbf{u}=0 \text { in } \mathcal{O}, \quad \mathbf{D}(\mathbf{u})=0 \text { in } B(\boldsymbol{\zeta})\right\}
$$

where $\boldsymbol{\zeta} \in \mathbb{R}^{2}$ and $B(\boldsymbol{\zeta})=\left\{\mathbf{x} \in \mathbb{R}^{2},|\mathbf{x}-\boldsymbol{\zeta}| \leqslant 1\right\}$. According to Lemma 1.1 of [29, pp.18], for any $\mathbf{u} \in \mathcal{K}(\boldsymbol{\zeta})$, there exist $\mathbf{l}_{\mathbf{u}} \in \mathbb{R}^{2}$ and $\omega_{\mathbf{u}} \in \mathbb{R}$ such that

$$
\mathbf{u}(\mathbf{y})=\mathbf{l}_{\mathbf{u}}+\omega_{\mathbf{u}}(\mathbf{y}-\boldsymbol{\zeta})^{\perp} \quad \forall \mathbf{y} \in B(\boldsymbol{\zeta}) .
$$

These spaces are specific to our problem. In fact, if the solution $\mathbf{u}$ of (1.1)-(1.8) is extended by

$$
\mathbf{u}(\mathbf{x}, t)=\boldsymbol{\zeta}^{\prime}(t)+\omega(t)(\mathbf{x}-\boldsymbol{\zeta}(t))^{\perp} \quad \forall \mathbf{x} \in B(\boldsymbol{\zeta}(t))
$$


then, we easily see that $\mathbf{u}(t) \in \widehat{\mathcal{K}}(\boldsymbol{\zeta}(t))$.

In all the sequel, the solution $\mathbf{u}$ of (1.1)-(1.8) will be extended as above.

We also notice that, by using (1.10), for any $\mathbf{u}, \mathbf{v} \in \mathcal{K}(\boldsymbol{\zeta})$ we have

$$
(\mathbf{u}, \mathbf{v})=\int_{\mathcal{O} \backslash B(\boldsymbol{\zeta})} \mathbf{u} \cdot \mathbf{v} \mathrm{d} \mathbf{x}+M \mathbf{l}_{\mathbf{u}} \cdot \mathbf{l}_{\mathbf{v}}+J \omega_{\mathbf{u}} \omega_{\mathbf{v}}
$$

Remark 2.1. In the case of different densities $\rho_{F} \neq \rho_{S}$, the natural inner product to be used seems to be

$$
<\mathbf{u}, \mathbf{v}>_{\zeta}=\rho_{F} \int_{\mathcal{O} \backslash B(\boldsymbol{\zeta})} \mathbf{u} \cdot \mathbf{v} \mathrm{d} \mathbf{x}+M \mathbf{l}_{\mathbf{u}} \cdot \mathbf{l}_{\mathbf{v}}+J \omega_{\mathbf{u}} \omega_{\mathbf{v}}
$$

which clearly depends on the position of the ball. This fact would considerably complicate the further analysis.

An important ingredient of the numerical method we use is given by the characteristic functions whose level lines are the integral curves of the velocity field. More precisely (see, for instance, [23], [26]) the characteristic function $\widetilde{\boldsymbol{\psi}}:[0, T]^{2} \times \mathcal{O} \rightarrow \mathcal{O}$ is defined as the solution of the initial value problem

$$
\left\{\begin{array}{l}
\frac{d}{d t} \widetilde{\boldsymbol{\psi}}(t ; s, \mathbf{x})=\mathbf{u}(\widetilde{\boldsymbol{\psi}}(t ; s, \mathbf{x}), t) \\
\widetilde{\boldsymbol{\psi}}(s ; s, \mathbf{x})=\mathbf{x}
\end{array}\right.
$$

It is well-known that the material derivative $D_{t} \mathbf{u}=\partial \mathbf{u} / \partial t+(\mathbf{u} \cdot \nabla) \mathbf{u}$ of $\mathbf{u}$ at instant $t_{0}$ satisfies:

$$
D_{t} \mathbf{u}\left(\mathbf{x}, t_{0}\right)=\frac{d}{d t}\left[\mathbf{u}\left(\widetilde{\boldsymbol{\psi}}\left(t ; t_{0}, \mathbf{x}\right), t\right)\right]_{\mid t=t_{0}} .
$$

Remark 2.2. By using a classical result of Liouville (see, for instance, Arnold [1, pp.251]), if

$$
\boldsymbol{\zeta} \in \mathcal{H}^{2}(0, T), \quad \omega \in H^{1}(0, T), \quad \mathbf{u} \in C([0, T] ; \widehat{\mathcal{K}}(\boldsymbol{\zeta}(t)))
$$

then, we have that

$$
\operatorname{det} \mathbf{J}_{\widetilde{\psi}}=1
$$

where we have denoted by

$$
\mathbf{J}_{\widetilde{\psi}}=\left(\frac{\partial \widetilde{\psi}_{i}}{\partial y_{j}}\right)_{i, j}
$$

the jacobian matrix of the transformation $\mathbf{y} \mapsto \widetilde{\psi}(\mathbf{y})$. 


\subsection{Weak form and semi-discretization scheme.}

In this subsection we give a weak form of (1.1)-(1.8) which is then used to discretize the problem with respect to time.

The fact that equation (2.9) below is called "weak formulation" of the system (1.1)-(1.8) is justified by the following result.

Lemma 2.3. Assume that

$$
\begin{gathered}
\mathbf{u} \in L^{2}\left(0, T ; \mathcal{H}^{2}(\Omega(t))\right) \\
p H^{1}\left(0, T ; \mathcal{L}^{2}(\Omega(t))\right) \cap C\left([0, T] ; \mathcal{H}^{1}(\Omega(t))\right), \\
p \in L^{2}\left(0, T ; H^{1}(\Omega(t))\right), \\
\boldsymbol{\zeta} \in \mathcal{H}^{2}(0, T), \quad \omega \in H^{1}(0, T)
\end{gathered}
$$

and that $\mathbf{u}$ is extended by

$$
\mathbf{u}(\mathbf{x}, t)=\boldsymbol{\zeta}^{\prime}(t)+\omega(t)(\mathbf{x}-\boldsymbol{\zeta}(t))^{\perp} \quad \forall \mathbf{x} \in B(\boldsymbol{\zeta}(t)) .
$$

Then $(\mathbf{u}, p, \boldsymbol{\zeta}, \omega)$ is the solution of (1.1)-(1.8) if and only if $\mathbf{u}(t) \in \widehat{\mathcal{K}}(\boldsymbol{\zeta}(t))$ for all $t$ and $(\mathbf{u}, p)$ satisfies

$$
\begin{aligned}
&\left(\frac{d}{d t}[\mathbf{u} \circ \tilde{\boldsymbol{\psi}}](t), \boldsymbol{\varphi}\right)+2 \nu(\mathbf{D}(\mathbf{u}(t)), \mathbf{D}(\boldsymbol{\varphi}))-\int_{\Omega(t)}(\operatorname{div} \boldsymbol{\varphi}) p(t) \mathrm{d} \mathbf{x} \\
&=(\mathbf{f}(t), \boldsymbol{\varphi}) \quad \forall \boldsymbol{\varphi} \in \mathcal{K}(\boldsymbol{\zeta}(t)) .
\end{aligned}
$$

We skip the proof of Lemma 2.3 since it is similar to the proof of the corresponding result for the classical Navier-Stokes system (see, for instance, [24, Ch.12]).

Remark 2.4. In the case of different densities $\rho_{F} \neq \rho_{S}$, a similar weak statement can be obtained (see for instance, [5]). In this case $\mathbf{u}$ in the first term of (2.9) should be replaced by $\rho \mathbf{u}$ where $\rho=\rho_{F}$ in the fluid and $\rho=\rho_{S}$ in the moving solid. Thus $\rho$ would depend on the time and a transport equation for $\rho$ should be added to the system.

By using the weak formulation given above we can derive a semi-discrete version of our system. For $N \in \mathbb{N}^{*}$ we denote $\Delta t=T / N$ and $t_{k}=k \Delta t$ for $k=0, \cdots, N$. Denote by $\left(\mathbf{u}^{k}, \boldsymbol{\zeta}^{k}\right) \in \widehat{\mathcal{K}}\left(\boldsymbol{\zeta}^{k}\right) \times \mathbb{R}^{2}$ the approximation of the solution of (1.1)-(1.8) at the time $t=t_{k}$. We approximate the position of the rigid ball at instant $t_{k+1}$ by $\zeta^{k+1}$ which is defined by the relation

$$
\boldsymbol{\zeta}^{k+1}=\boldsymbol{\zeta}^{k}+\mathbf{u}^{k}\left(\boldsymbol{\zeta}^{k}\right) \Delta t .
$$

We then define characteristic function $\bar{\psi}$ associated to the semi-discretized velocity field as the solution of

$$
\left\{\begin{array}{l}
\frac{d}{d t} \overline{\boldsymbol{\psi}}\left(t ; t_{k+1}, \mathbf{x}\right)=\mathbf{u}^{k}\left(\overline{\boldsymbol{\psi}}\left(t ; t_{k+1}, \mathbf{x}\right)\right) \\
\overline{\boldsymbol{\psi}}\left(t_{k+1} ; t_{k+1}, \mathbf{x}\right)=\mathbf{x}
\end{array}\right.
$$

and we denote

$$
\overline{\boldsymbol{X}}^{\boldsymbol{k}}(\mathbf{x})=\overline{\boldsymbol{\psi}}\left(t_{k} ; t_{k+1}, \mathbf{x}\right) \quad \forall \mathbf{x} \in \mathcal{O} .
$$

One can easily check that $\overline{\boldsymbol{X}}^{\boldsymbol{k}}(\mathcal{O})=\mathcal{O}$. 
We next define $\mathbf{u}^{k+1} \in \widehat{\mathcal{K}}\left(\boldsymbol{\zeta}^{k+1}\right)$ as the solution of the following Stokes type system

$$
\left(\frac{\mathbf{u}^{k+1}-\mathbf{u}^{k} \circ \overline{\boldsymbol{X}}^{\boldsymbol{k}}}{\Delta t}, \boldsymbol{\varphi}\right)+2 \nu\left(\mathbf{D}\left(\mathbf{u}^{k+1}\right), \mathbf{D}(\boldsymbol{\varphi})\right)=\left(\mathbf{f}^{k+1}, \boldsymbol{\varphi}\right) \quad \forall \boldsymbol{\varphi} \in \widehat{\mathcal{K}}\left(\boldsymbol{\zeta}^{k+1}\right),
$$

where $\mathbf{f}^{k+1}=\mathbf{f}\left(t_{k+1}\right)$.

The above equation can be rewritten by using a mixed formulation. To achieve this, we first define:

$$
\begin{gathered}
M(\boldsymbol{\zeta})=\left\{p \in L_{0}^{2}(\mathcal{O}) \mid p=0 \text { in } B(\boldsymbol{\zeta})\right\} \\
a(\mathbf{u}, \mathbf{v})=2 \nu \int_{\mathcal{O}} \mathbf{D}(\mathbf{u}): \mathbf{D}(\mathbf{v}) \mathrm{d} \mathbf{x} \quad \forall \mathbf{u}, \mathbf{v} \in \mathcal{H}^{1}(\mathcal{O}), \\
b(\mathbf{u}, p)=-\int_{\mathcal{O}} \operatorname{div}(\mathbf{u}) p \mathrm{~d} \mathbf{x} \quad \forall \mathbf{u} \in \mathcal{H}^{1}(\mathcal{O}) \quad \forall p \in L_{0}^{2}(\mathcal{O}) .
\end{gathered}
$$

With the above notation, it is clear that (2.13) is equivalent to the system

$$
\begin{aligned}
& \qquad \begin{array}{l}
\left(\frac{\mathbf{u}^{k+1}-\mathbf{u}^{k} \circ \overline{\boldsymbol{X}}^{\boldsymbol{k}}}{\Delta t}, \boldsymbol{\varphi}\right)+a\left(\mathbf{u}^{k+1}, \boldsymbol{\varphi}\right)+b\left(\boldsymbol{\varphi}, p^{k+1}\right)=\left(\mathbf{f}^{k+1}, \boldsymbol{\varphi}\right) \quad \forall \boldsymbol{\varphi} \in \mathcal{K}\left(\boldsymbol{\zeta}^{k+1}\right), \\
b\left(\mathbf{u}^{k+1}, q\right)=0 \quad \forall q \in M\left(\boldsymbol{\zeta}^{k+1}\right),
\end{array} \\
& \text { of unknowns }\left(\mathbf{u}^{k+1}, p^{k+1}\right) \in \mathcal{K}\left(\boldsymbol{\zeta}^{k+1}\right) \times M\left(\boldsymbol{\zeta}^{k+1}\right) .
\end{aligned}
$$

Remark 2.5. The requirement $p=0$ in $B(\boldsymbol{\zeta})$ for the definition of $M(\boldsymbol{\zeta})$ allows us to define the form $b$ on the whole domain $\mathcal{O}$. This extension does not affect the form $b$ since $\operatorname{div}(\mathbf{u})=0$ in $B(\boldsymbol{\zeta})$ for all $\mathbf{u} \in \mathcal{K}(\boldsymbol{\zeta})$.

It is well-known (see, for example, [12, Corollary I.4.1., pp.61]) that the mixed formulation $(2.17),(2.18)$ is a well-posed problem, provided that the spaces $\mathcal{K}(\boldsymbol{\zeta}), M(\boldsymbol{\zeta})$ and the bilinear form $b$ satisfy an inf-sup condition. The fact that this inf-sup condition is satisfied in our case follows from the result below.

Lemma 2.6. Suppose that $\boldsymbol{\zeta} \in \mathcal{O}$ is such that $d(\boldsymbol{\zeta}, \partial \mathcal{O})=1+\eta$, with $\eta>0$. Then there exists a constant $\beta>0$, depending only on $\eta$ and on $\mathcal{O}$, such that for all $q \in M(\boldsymbol{\zeta})$ there exists $\mathbf{u} \in \mathcal{K}(\boldsymbol{\zeta})$ with

$$
\int_{\mathcal{O}} \operatorname{div}(\mathbf{u}) q \mathrm{~d} \mathbf{x} \geq \beta\|\mathbf{u}\|_{\mathcal{H}^{1}(\mathcal{O})}\|q\|_{L^{2}(\mathcal{O})}
$$

The proof of the result above can be obtained by slightly modifying the approach used for the mixed formulation of the standard Stokes system (see, for instance [12, pp.81]), so it is left to the reader. 


\section{Full discretization and statement of the main result.}

In order to discretize the problem (2.17), (2.18) with respect to the space variable we introduce two families of finite element spaces. We first define a family of finite element spaces which approximate the space $\mathcal{K}(\boldsymbol{\zeta})$ defined in (2.3). Let $h$ denote a discretization parameter, $0<h<1$ and let $P_{1}$ be the space of all affine functions in $\mathbb{R}^{2}$.

Consider a quasi-uniform triangulation $\mathcal{T}_{h}$ of $\mathcal{O}$, as defined, for instance, in [2, p.106] (this assumption will be accepted in the remaining part of this paper and will allows us to make use of inverse estimates). If $T \in \mathcal{T}_{h}$ is a triangle of vertices $\mathbf{x}_{1}, \mathbf{x}_{2}, \mathbf{x}_{3}$ we denote by $\varphi_{1}(\mathbf{x}), \varphi_{2}(\mathbf{x})$ and $\varphi_{3}(\mathbf{x})$ the corresponding barycentric coordinates of $\mathbf{x} \in \mathbb{R}^{2}$ with respect to the vertices $\mathbf{x}_{1}, \mathbf{x}_{2}, \mathbf{x}_{3}$ (see, for instance, [4, p.45] for the definition of barycentric coordinates). We associate to this triangulation two classical approximation spaces used in the mixed finite element methods for the Stokes system. The first space, classically used for the approximation of the velocity field in the mixed statement of the Stokes system, is denoted by $\mathcal{W}_{h}$ and is defined as the subspace of $\mathcal{H}_{0}^{1}(\mathcal{O})$ formed by the $P_{1}$-bubble finite elements associated to $\mathcal{T}_{h}$. More precisely $\varphi \in \mathcal{W}_{h}$ if and only if

$$
\boldsymbol{\varphi}(\mathbf{x})=\varphi_{1}(\mathbf{x}) \boldsymbol{\alpha}_{\mathbf{1}}+\varphi_{2}(\mathbf{x}) \boldsymbol{\alpha}_{\mathbf{2}}+\varphi_{3}(\mathbf{x}) \boldsymbol{\alpha}_{\mathbf{3}}+\frac{\varphi_{1}(\mathbf{x}) \varphi_{2}(\mathbf{x}) \varphi_{3}(\mathbf{x})}{\int_{T} \varphi_{1} \varphi_{2} \varphi_{3} \mathrm{~d} \mathbf{x}} \boldsymbol{\lambda} \quad \forall \mathbf{x} \in T
$$

for some constant vectors $\boldsymbol{\alpha}_{\mathbf{1}}, \boldsymbol{\alpha}_{\mathbf{2}}, \boldsymbol{\alpha}_{\mathbf{3}}, \boldsymbol{\lambda} \in \mathbb{R}^{2}$. We may notice that all functions in $\mathcal{W}_{h}$ are continuous.

The second space, classically used for the approximation of the pressure in mixed statement of the Stokes system, is denoted by $E_{h}$ and is defined by

$$
E_{h}=\left\{q \in C(\overline{\mathcal{O}}) \mid q_{\mid T} \in P_{1}(T)\right\} .
$$

For our problem we use two spaces which are related to the presence of the rigid body. The first one, which is used for the approximation of the velocity field is denoted by $\mathcal{K}_{h}(\boldsymbol{\zeta})$ and defined by

$$
\mathcal{K}_{h}(\boldsymbol{\zeta})=\mathcal{W}_{h} \cap \mathcal{K}(\boldsymbol{\zeta}) \quad \forall \boldsymbol{\zeta} \in \mathcal{O}
$$

The second one, which is used for the approximation of the pressure, is denoted by $M_{h}(\boldsymbol{\zeta})$ and is defined by

$$
M_{h}(\boldsymbol{\zeta})=E_{h} \cap M(\boldsymbol{\zeta}) \quad \forall \boldsymbol{\zeta} \in \mathcal{O} .
$$

We also define the finite element space (see [23])

$$
\mathcal{R}_{h}=\left\{\operatorname{rot} \varphi_{h}, \quad \varphi_{h} \in E_{h}, \quad \varphi_{h}=0 \text { on } \partial \mathcal{O}\right\}
$$

We denote by $\mathbf{P}$ the orthogonal projection from $\mathcal{L}^{2}$ onto $\mathcal{R}_{h}$. More precisely if $\mathbf{u} \in \mathcal{L}^{2}(\mathcal{O})$ then $\mathbf{P u} \in \mathcal{R}_{h}$ satisfies

$$
\left(\mathbf{u}-\mathbf{P u}, \mathbf{r}_{h}\right)=0 \quad \forall \mathbf{r}_{h} \in \mathcal{R}_{h} .
$$

Let $N$ be a positive integer. We denote $\Delta t=T / N$ and $t_{k}=k \Delta t$. Assume that the approximate solution $\left(\mathbf{u}_{h}^{k}, p_{h}^{k}, \boldsymbol{\zeta}_{h}^{k}\right)$ of (1.1)-(1.8) at $t=t_{k}$ is known. We describe below the 
numerical scheme allowing to determinate the approximate solution $\left(\mathbf{u}_{h}^{k+1}, p_{h}^{k+1}, \boldsymbol{\zeta}_{h}^{k+1}\right)$ at $t=t_{k+1}$. First, we compute $\boldsymbol{\zeta}_{h}^{k+1} \in \mathbb{R}^{2}$ by

$$
\boldsymbol{\zeta}_{h}^{k+1}=\boldsymbol{\zeta}_{h}^{k}+\mathbf{u}_{h}^{k}\left(\boldsymbol{\zeta}_{h}^{k}\right) \Delta t
$$

We denote by $\mathbf{P} \mathbf{u}_{h}^{k}$ the projection of $\mathbf{u}_{h}^{k}$ onto $\mathcal{R}_{h}$. Then, we define the characteristic function $\overline{\boldsymbol{\psi}}_{\boldsymbol{h}}^{k}$ associated to the fully discretized velocity field as the solution of

$$
\left\{\begin{array}{l}
\frac{d}{d t} \overline{\boldsymbol{\psi}}_{\boldsymbol{h}}^{\boldsymbol{k}}\left(t ; t_{k+1}, \mathbf{x}\right)=\mathbf{P u}_{h}^{k}\left(\overline{\boldsymbol{\psi}}_{\boldsymbol{h}}^{\boldsymbol{k}}\left(t ; t_{k+1}, \mathbf{x}\right)\right), \\
\overline{\boldsymbol{\psi}}_{\boldsymbol{h}}^{\boldsymbol{k}}\left(t_{k+1} ; t_{k+1}, \mathbf{x}\right)=\mathbf{x}
\end{array}\right.
$$

We also define

$$
\overline{\boldsymbol{X}}_{\boldsymbol{h}}^{\boldsymbol{k}}(\mathbf{x})=\overline{\boldsymbol{\psi}}_{\boldsymbol{h}}^{\boldsymbol{k}}\left(t_{k} ; t_{k+1}, \mathbf{x}\right) \quad \forall \mathbf{x} \in \mathcal{O}
$$

and as for the problem (2.11), one can check that $\overline{\boldsymbol{X}}_{\boldsymbol{h}}^{\boldsymbol{k}}(\mathcal{O})=\mathcal{O}$ (see Remark 3.1 below).

Then, we define $\left(\mathbf{u}_{h}^{k+1}, p_{h}^{k+1}\right) \in \mathcal{K}_{h}\left(\boldsymbol{\zeta}_{h}^{k+1}\right) \times M_{h}\left(\boldsymbol{\zeta}_{h}^{k+1}\right)$ as the solution of the problem:

$$
\begin{gathered}
\left(\frac{\mathbf{u}_{h}^{k+1}-\mathbf{u}_{h}^{k} \circ \overline{\boldsymbol{X}}_{\boldsymbol{h}}^{\boldsymbol{k}}}{\Delta t}, \boldsymbol{\varphi}\right)+a\left(\mathbf{u}_{h}^{k+1}, \boldsymbol{\varphi}\right)+b\left(\boldsymbol{\varphi}, p_{h}^{k+1}\right)=\left(\mathbf{f}_{h}^{k+1}, \boldsymbol{\varphi}\right) \quad \forall \boldsymbol{\varphi} \in \mathcal{K}_{h}\left(\boldsymbol{\zeta}_{h}^{k+1}\right), \\
b\left(\mathbf{u}_{h}^{k+1}, q\right)=0 \quad \forall q \in M_{h}\left(\boldsymbol{\zeta}_{h}^{k+1}\right),
\end{gathered}
$$

where $\mathbf{f}_{h}^{k+1}$ is the $\mathcal{L}^{2}$-projection of $\mathbf{f}^{k+1}=\mathbf{f}\left(t_{k+1}\right)$ on $\left(E_{h}\right)^{2}$. We take $\boldsymbol{\zeta}_{h}^{0}=\boldsymbol{\zeta}^{0}$ and the initial approximate velocity $\mathbf{u}_{h}^{0}$ is the $\mathcal{H}_{0}^{1}$-projection of $\mathbf{u}_{0}$ onto $\mathcal{K}_{h}\left(\boldsymbol{\zeta}_{h}^{0}\right)$.

Remark 3.1. In (3.3), we use the projection of $\mathbf{u}_{h}^{k}$ on $\mathcal{R}_{h}$ rather than the function $\mathbf{u}_{h}^{k}$ itself because $\operatorname{div}\left(\mathbf{P} \mathbf{u}_{h}^{k}\right)=0$ in $\mathcal{O}$. By using a classical result of Liouville, this implies that $\operatorname{det} \mathbf{J}_{\overline{\boldsymbol{\psi}}_{h}^{k}}=1$ and in particular that $\operatorname{det} \mathbf{J}_{\overline{\boldsymbol{X}}_{h}^{k}}=1$. This property combined to the fact that the velocity field $\mathbf{P} \mathbf{u}_{h}^{k}$ vanishes along the boundary $\partial \mathcal{O}$ entails the invariance property of the whole domain $\mathcal{O}$ through $\overline{\boldsymbol{X}}_{\boldsymbol{h}}^{\boldsymbol{k}}$ i.e. $\overline{\boldsymbol{X}}_{\boldsymbol{h}}^{\boldsymbol{k}}(\mathcal{O})=\mathcal{O}$. Moreover, since $\mathbf{P} \mathbf{u}_{h}^{k}$ is constant in each triangle, the initial value problem (3.3) can be solved exactly.

In the sequel, we suppose that

$$
\begin{aligned}
& \mathbf{f} \in C\left([0, T] ; \mathcal{H}^{1}(\mathcal{O})\right), \quad \mathbf{u}_{0} \in \mathcal{H}^{2}(\Omega), \quad \operatorname{div}\left(\mathbf{u}_{0}\right)=0 \quad \text { in } \Omega, \\
& \mathbf{u}_{0}=0 \quad \text { on } \partial \mathcal{O}, \quad \mathbf{u}_{0}(\mathbf{y})=\boldsymbol{\zeta}_{1}+\omega_{0}\left(\mathbf{y}-\boldsymbol{\zeta}_{0}\right)^{\perp} \quad \text { on } \partial B .
\end{aligned}
$$

The corresponding solution $(\mathbf{u}, p, \boldsymbol{\zeta}, \omega)$ of problem (1.1)-(1.8) will be assumed to satisfy the following regularity hypotheses

$$
\left\{\begin{array}{l}
\mathbf{u} \in C\left([0, T] ; \mathcal{H}^{2}(\Omega(t))\right) \cap H^{1}\left(0, T ; \mathcal{L}^{2}(\Omega(t))\right), \\
D_{t}^{2} \mathbf{u} \in L^{2}\left(0, T ; \mathcal{L}^{2}(\Omega(t))\right), \quad \mathbf{u} \in C\left([0, T] ; \mathcal{C}^{0,1}(\overline{\mathcal{O}})\right) \\
p \in C\left([0, T] ; \mathcal{H}^{1}(\Omega(t))\right), \quad \boldsymbol{\zeta} \in \mathcal{H}^{3}(0, T), \quad \omega \in H^{2}(0, T) .
\end{array}\right.
$$

Moreover, we assume that

$$
\operatorname{dist}(B(t), \partial \mathcal{O})>0 \quad \forall t \in[0, T] .
$$

The hypotheses (3.8) and (3.9) imply the existence of $\eta>0$ such that

$$
\operatorname{dist}(B(t), \partial \mathcal{O})>3 \eta \quad \forall t \in[0, T] .
$$


Theorem 3.2. Let $C_{0}>0$ be a fixed constant. Suppose that $\mathcal{O}$ is the interior of a convex polygon and that $(\mathbf{u}, p, \boldsymbol{\zeta}, \omega)$ is a solution of (1.1)-(1.8) satisfying (3.8) and (3.9). Moreover, assume that $\mathbf{f}$ and $\mathbf{u}_{0}$ satisfy (3.7). Consider the functions $\boldsymbol{\zeta}_{h}^{k}$, $\mathbf{u}_{h}^{k}$ and $p_{h}^{k}$ defined in this section. Then there exist two positive constants $C$ and $\tau^{*}$ not depending on $h$ and on $\Delta t$ such that for all $0<\Delta t \leqslant \tau^{*}$ and for all $h \leqslant C_{0}(\Delta t)^{2}$ we have

$$
\sup _{1 \leqslant k \leqslant N}\left(\left|\boldsymbol{\zeta}\left(t_{k}\right)-\boldsymbol{\zeta}_{h}^{k}\right|+\left\|\mathbf{u}\left(t_{k}\right)-\mathbf{u}_{h}^{k}\right\|_{\mathcal{L}^{2}(\mathcal{O})}\right) \leqslant C \Delta t .
$$

Remark 3.3. For the Navier-Stokes system, the same type of result is obtained in [23] for $h \leqslant C_{0} \Delta t$ and in [26] for $h^{2} \leqslant C_{0} \Delta t \leqslant C_{1} h^{\sigma}$ and $\sigma>1 / 2$ (for $h$ and $\Delta t$ small enough).

Remark 3.4. It can be easily checked, by using the fact that $\operatorname{det} \mathbf{J}_{\bar{\psi}_{h}^{k}}=1$, that our method is unconditionally stable.

\section{Some properties of the finite element spaces.}

We next give some technical results on the finite element spaces introduced above. Throughout this section we consider $\boldsymbol{\zeta} \in \mathcal{O}$ such that $\operatorname{dist}(B(\boldsymbol{\zeta}), \partial \mathcal{O})>2 \eta$ and we suppose that $h<\eta$. Therefore, we have that

$$
\operatorname{dist}(B(\boldsymbol{\zeta}), \partial \mathcal{O})>2 h
$$

Notice that, by definition, if $q \in M_{h}(\boldsymbol{\zeta})$ then $q=0$ in $B(\boldsymbol{\zeta})$. Since $q$ is a $P_{1}$ function in each triangle it follows that $q_{\mid A_{h}}=0$, where

$$
A_{h}=\bigcup_{\substack{T \in \mathcal{T}_{h} \\ \stackrel{\circ}{\circ} \dot{\circ}(\boldsymbol{\zeta}) \neq \emptyset}} T .
$$

Moreover, if we denote by $Q_{h}$ the union of all triangles $T \in \mathcal{T}_{h}$ such that the three vertices of $T$ are contained in $\overline{A_{h}}$ then, by using again the fact that $q$ is a $P_{1}$ function in each triangle, it follows that

$$
q_{\mid Q_{h}}=0 \quad \forall q \in M_{h}(\boldsymbol{\zeta})
$$

A similar argument shows that

$$
\mathbf{D}(\mathbf{u})_{\mid A_{h}}=0 \quad \forall \mathbf{u} \in \mathcal{K}_{h}(\boldsymbol{\zeta})
$$

In order to study the properties of the spaces $\mathcal{K}_{h}(\boldsymbol{\zeta})$ and $M_{h}(\boldsymbol{\zeta})$ defined above we divide the triangles in $\mathcal{T}_{h}$ in four categories. These categories are defined as follows (see figure $1)$ :

- $\mathcal{F}_{1}$ is the subset of $\mathcal{T}_{h}$ formed by all triangles $T \in \mathcal{T}_{h}$ such that $\bar{T} \subset B(\boldsymbol{\zeta})$.

- $\mathcal{F}_{2}$ is the subset formed by all triangles $T \in \mathcal{T}_{h} \backslash \mathcal{F}_{1}$ such that $\bar{T} \subset \bar{Q}_{h}$. 


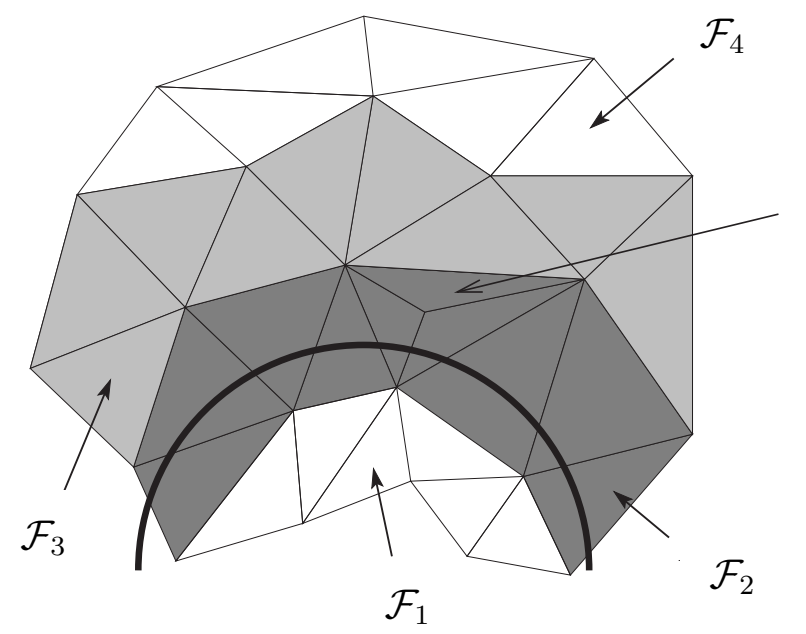

This kind of triangles are not included in $A_{h}$ since $T \cap B=\emptyset$.

But it's included in $Q_{h}$ since its three vertices are in $\overline{A_{h}}$.

Figure 1: Splitting of the triangulation into four families of triangles.

- $\mathcal{F}_{3}$ is the subset formed by all triangles $T \in \mathcal{T}_{h}$ such that $\bar{T} \cap \overline{Q_{h}} \neq \emptyset$ and $T \not \subset \overline{Q_{h}}$.

- $\mathcal{F}_{4}=\mathcal{T}_{h} \backslash\left(\mathcal{F}_{1} \cup \mathcal{F}_{2} \cup \mathcal{F}_{3}\right)$.

Lemma 4.1. There exists a positive constant $C_{1}$ (not depending on the position of $B(\boldsymbol{\zeta})$ ) such that

$$
\begin{aligned}
& \inf _{\mathbf{v}_{h} \in \mathcal{K}_{h}(\boldsymbol{\zeta})}\left\|\mathbf{v}-\mathbf{v}_{h}\right\|_{L^{2}(\mathcal{O})} \leqslant C_{1} h^{\frac{3}{2}}\left(\|\mathbf{v}\|_{\mathcal{H}^{2}(\mathcal{O} \backslash B(\zeta))}+\|\mathbf{v}\|_{\mathcal{H}^{2}(B(\zeta))}\right), \\
& \inf _{\mathbf{v}_{h} \in \mathcal{K}_{h}(\boldsymbol{\zeta})}\left\|\mathbf{v}-\mathbf{v}_{h}\right\|_{\mathcal{H}^{1}(\mathcal{O})} \leqslant C_{1} \sqrt{h}\left(\|\mathbf{v}\|_{\mathcal{H}^{2}(\mathcal{O} \backslash B(\zeta))}+\|\mathbf{v}\|_{\mathcal{H}^{2}(B(\zeta))}\right),
\end{aligned}
$$

for all $\mathbf{v} \in \mathcal{K}(\boldsymbol{\zeta}) \cap \mathcal{H}^{2}(\mathcal{O} \backslash B(\boldsymbol{\zeta}))$.

Proof. Let $\mathbf{v} \in \mathcal{K}(\boldsymbol{\zeta}) \cap \mathcal{H}^{2}(\mathcal{O} \backslash B(\boldsymbol{\zeta}))$. This means, in particular, that

$$
\mathbf{v}(\mathbf{x})=\mathbf{l}+\omega \mathbf{x}^{\perp} \quad \forall \mathbf{x} \in B(\boldsymbol{\zeta}),
$$

for some $\mathbf{l} \in \mathbb{R}^{2}$ and $\omega \in \mathbb{R}$. In the remaining part of this section we denote

$$
\mathbf{R}(\mathbf{x})=\mathbf{l}+\omega \mathbf{x}^{\perp} \quad \forall \mathbf{x} \in \mathbb{R}^{2} .
$$

We denote by $\mathbf{v}_{I h}$ the unique function in $\left(E_{h}\right)^{2}$ which agrees with $\mathbf{v}$ at every node $\mathbf{x}_{j}$ of the triangulation $\mathcal{T}_{h}$ (recall the definition of $E_{h}$ in (3.1)). Then we consider the function $\mathbf{v}_{h} \in\left(E_{h}\right)^{2}$ whose value in a node $\mathbf{x}_{\mathbf{j}}$ of $\mathcal{T}_{h}$ is defined by

$$
\mathbf{v}_{\mathbf{h}}\left(\mathbf{x}_{\mathbf{j}}\right)=\left\{\begin{array}{lll}
\mathbf{R}\left(\mathbf{x}_{\mathbf{j}}\right) & \text { if } & \mathbf{x}_{\mathbf{j}} \in \overline{A_{h}} \\
\mathbf{v}_{I h}\left(\mathbf{x}_{\mathbf{j}}\right) & \text { if } & \mathbf{x}_{\mathbf{j}} \notin \overline{A_{h}}
\end{array}\right.
$$

Since $\mathbf{v}_{\mathbf{h}}$ is affine in each triangle, it follows that

$$
\mathbf{v}_{\mathbf{h}}(\mathbf{x})=\mathbf{R}(\mathbf{x}) \quad \forall \mathbf{x} \in \overline{Q_{h}} .
$$


We will show that there exists a positive constant $C_{1}$ (not depending on the position of $B(\boldsymbol{\zeta}))$ such that

$$
\begin{aligned}
& \left\|\mathbf{v}-\mathbf{v}_{h}\right\|_{\mathcal{L}^{2}(\mathcal{O})} \leqslant C_{1} h^{\frac{3}{2}}\left(\|\mathbf{v}\|_{\mathcal{H}^{2}(\mathcal{O} \backslash B(\boldsymbol{\zeta}))}+\|\mathbf{v}\|_{\mathcal{H}^{2}(B(\boldsymbol{\zeta}))}\right) \\
& \left\|\mathbf{v}-\mathbf{v}_{h}\right\|_{\mathcal{H}^{1}(\mathcal{O})} \leqslant C_{1} \sqrt{h}\left(\|\mathbf{v}\|_{\mathcal{H}^{2}(\mathcal{O} \backslash B(\boldsymbol{\zeta}))}+\|\mathbf{v}\|_{\mathcal{H}^{2}(B(\boldsymbol{\zeta}))}\right) .
\end{aligned}
$$

In order to prove the above inequalities, we divide the domain $\mathcal{O}$ into four parts:

$$
\mathcal{O}=B(\boldsymbol{\zeta}) \cup\left(Q_{h} \backslash B(\boldsymbol{\zeta})\right) \cup\left(\bigcup_{T \in \mathcal{F}_{3}} T\right) \cup\left(\bigcup_{T \in \mathcal{F}_{4}} T\right)
$$

Let us first remark that

$$
\mathbf{v}=\mathbf{R} \text { in } B(\boldsymbol{\zeta})
$$

On the other hand it is clear that $Q_{h}$ is contained in the closed ball of center $\boldsymbol{\zeta}$ and of radius $1+h$, denoted by $B_{h}(\boldsymbol{\zeta})$. Let us remark that the ball $B_{h}(\boldsymbol{\zeta})$ is included into the domain $\mathcal{O}$ due to the condition (4.1). According to a classical result (see, for instance, Lemma 5.11 in Fujita and Sauer [10]) there exists a universal constant $C>0$, such that for all $\boldsymbol{\varphi} \in \mathcal{H}^{1}(\mathcal{O} \backslash B(\boldsymbol{\zeta}))$,

$$
\|\boldsymbol{\varphi}\|_{\mathcal{L}^{2}\left(B_{h}(\boldsymbol{\zeta}) \backslash B(\boldsymbol{\zeta})\right)} \leq C\left(\sqrt{h}\|\boldsymbol{\varphi}\|_{\mathcal{L}^{2}(\partial B(\boldsymbol{\zeta}))}+h\|\nabla \boldsymbol{\varphi}\|_{\left[L^{2}\left(B_{h}(\boldsymbol{\zeta}) \backslash B(\boldsymbol{\zeta})\right)\right]^{4}}\right) .
$$

The above relation with $\boldsymbol{\varphi}=\mathbf{v}-\mathbf{R}$ and (4.5) imply that

$$
\|\mathbf{v}-\mathbf{R}\|_{\mathcal{L}^{2}\left(B_{h}(\boldsymbol{\zeta}) \backslash B(\boldsymbol{\zeta})\right)} \leqslant C h\|\nabla(\mathbf{v}-\mathbf{R})\|_{\left[L^{2}\left(B_{h}(\boldsymbol{\zeta}) \backslash B(\boldsymbol{\zeta})\right)\right]^{4}} .
$$

By applying again Lemma 5.11 in [10] (this time for the function $\nabla(\mathbf{v}-\mathbf{R})$ ) we obtain that

$$
\begin{aligned}
\|\nabla(\mathbf{v}-\mathbf{R})\|_{\left[L^{2}\left(B_{h}(\boldsymbol{\zeta}) \backslash B(\boldsymbol{\zeta})\right)\right]^{4}} \leqslant C\left(\sqrt{h}\|\nabla(\mathbf{v}-\mathbf{R})\|_{\left[L^{2}(\partial B(\boldsymbol{\zeta}))\right]^{4}}\right. & \\
& \left.+h\|\nabla(\mathbf{v}-\mathbf{R})\|_{\left[H^{1}(\mathcal{O} \backslash B(\boldsymbol{\zeta}))\right]^{4}}\right) .
\end{aligned}
$$

The above inequality, combined with the trace theorem in Sobolev spaces gives that

$$
\|\nabla(\mathbf{v}-\mathbf{R})\|_{\left[L^{2}\left(B_{h}(\boldsymbol{\zeta}) \backslash B(\boldsymbol{\zeta})\right)\right]^{4}} \leq C \sqrt{h}\|\mathbf{v}-\mathbf{R}\|_{\mathcal{H}^{2}(\mathcal{O} \backslash B(\boldsymbol{\zeta}))} .
$$

From (4.7) and (4.8) it follows that

$$
\|\mathbf{v}-\mathbf{R}\|_{\mathcal{L}^{2}\left(B_{h}(\boldsymbol{\zeta}) \backslash B(\boldsymbol{\zeta})\right)} \leqslant C h^{\frac{3}{2}}\|\mathbf{v}-\mathbf{R}\|_{\mathcal{H}^{2}(\mathcal{O} \backslash B(\boldsymbol{\zeta}))} .
$$

The above relation implies, by using the fact that $Q_{h} \subset B_{h}(\boldsymbol{\zeta})$ and (4.2), that

$$
\left\|\mathbf{v}-\mathbf{v}_{\mathbf{h}}\right\|_{\mathcal{L}^{2}\left(Q_{h} \backslash B(\boldsymbol{\zeta})\right)} \leq C h^{\frac{3}{2}}\|\mathbf{v}-\mathbf{R}\|_{\mathcal{H}^{2}(\mathcal{O} \backslash B(\boldsymbol{\zeta}))} .
$$

Consequently we have that

$$
\left\|\mathbf{v}-\mathbf{v}_{h}\right\|_{\mathcal{L}^{2}\left(Q_{h} \backslash B(\boldsymbol{\zeta})\right)} \leqslant C_{1} h^{\frac{3}{2}}\left(\|\mathbf{v}\|_{\mathcal{H}^{2}(\mathcal{O} \backslash B(\boldsymbol{\zeta}))}+\|\mathbf{v}\|_{\mathcal{H}^{2}(B(\boldsymbol{\zeta}))}\right) .
$$


On the other hand (4.8) and (4.9) imply that

$$
\|\mathbf{v}-\mathbf{R}\|_{\mathcal{H}^{1}\left(B_{h}(\boldsymbol{\zeta}) \backslash B(\boldsymbol{\zeta})\right)} \leq C h^{\frac{1}{2}}\|\mathbf{v}-\mathbf{R}\|_{\mathcal{H}^{2}(\mathcal{O} \backslash B(\boldsymbol{\zeta}))}
$$

The above relation implies, by using the fact that $Q_{h} \subset B_{h}(\boldsymbol{\zeta})$ and (4.2) that

$$
\left\|\mathbf{v}-\mathbf{v}_{\mathbf{h}}\right\|_{\mathcal{H}^{1}\left(Q_{h} \backslash B(\boldsymbol{\zeta})\right)} \leq C h^{\frac{1}{2}}\|\mathbf{v}-\mathbf{R}\|_{\mathcal{H}^{2}(\mathcal{O} \backslash B(\boldsymbol{\zeta}))},
$$

which clearly implies

$$
\left\|\mathbf{v}-\mathbf{v}_{\mathbf{h}}\right\|_{\mathcal{H}^{1}\left(Q_{h} \backslash B(\boldsymbol{\zeta})\right)} \leq C \sqrt{h}\left(\|\mathbf{v}\|_{\mathcal{H}^{2}(\mathcal{O} \backslash B(\boldsymbol{\zeta}))}+\|\mathbf{v}\|_{\mathcal{H}^{2}(B(\boldsymbol{\zeta}))}\right)
$$

Let us now consider a triangle $T \in \mathcal{F}_{3}$. In order to estimate the restriction of $\mathbf{v}-\mathbf{v}_{\mathbf{h}}$ to $T$ we use the interpolating function $\mathbf{v}_{I h}$. More precisely we have

$$
\left\|\mathbf{v}-\mathbf{v}_{h}\right\|_{\alpha} \leq\left\|\mathbf{v}-\mathbf{v}_{I h}\right\|_{\alpha}+\left\|\mathbf{v}_{I h}-\mathbf{v}_{h}\right\|_{\alpha}, \quad \alpha \in\{0,1\}
$$

where $\|\cdot\|_{\alpha}$ stands for the $\mathcal{L}^{2}$-norm or the $\mathcal{H}^{1}$-norm on $T$. We first estimate the second term in the right hand side of (4.13). Since the function $\mathbf{v}_{I h}-\mathbf{v}_{h}$ is affine in $T$ we have

$$
\mathbf{v}_{I h}(\mathbf{x})-\mathbf{v}_{h}(\mathbf{x})=\sum_{i=1}^{3}\left(\mathbf{v}_{I h}\left(\mathbf{x}_{i}\right)-\mathbf{v}_{h}\left(\mathbf{x}_{i}\right)\right) \varphi_{i}(x),
$$

where $\left(\mathbf{x}_{i}\right)$ are the nodes of $T$ and $\left(\varphi_{i}\right)$ are the corresponding Lagrange barycentric functions. We have

$$
\left\|\mathbf{v}_{I h}-\mathbf{v}_{h}\right\|_{\alpha} \leq \sum_{i=1}^{3}\left|\mathbf{v}_{I h}\left(\mathbf{x}_{i}\right)-\mathbf{v}_{h}\left(\mathbf{x}_{i}\right)\right|\left\|\varphi_{i}\right\|_{\alpha} .
$$

A simple calculation shows that

$$
\left\|\varphi_{i}\right\|_{L^{2}(T)} \leq C h
$$

and

$$
\left\|\nabla \varphi_{i}\right\|_{L^{2}(T)} \leq C
$$

Since the mesh is quasi-uniform the constant $C$ can be chosen not depending on the triangle. We now estimate $\left|\mathbf{v}_{I h}\left(\mathbf{x}_{i}\right)-\mathbf{v}_{h}\left(\mathbf{x}_{i}\right)\right|$. Since $T \not \subset Q_{h}$ it follows that $T$ has at most two nodes in $Q_{h}$ and, consequently, at least one node such that $\mathbf{v}_{I h}\left(\mathbf{x}_{i}\right)-\mathbf{v}_{h}\left(\mathbf{x}_{i}\right)=0$. Therefore we tackle only the nodes in $Q_{h}$. If $\mathbf{x}_{i}$ is a node in $Q_{h}$ then

$$
\left|\mathbf{v}_{I h}\left(\mathbf{x}_{i}\right)-\mathbf{v}_{h}\left(\mathbf{x}_{i}\right)\right|=\left|\mathbf{v}\left(\mathbf{x}_{i}\right)-\mathbf{R}\left(\mathbf{x}_{i}\right)\right|
$$

Relations (4.14), (4.15) and (4.17) imply that

$$
\begin{aligned}
\left\|\mathbf{v}_{I h}-\mathbf{v}_{h}\right\|_{\mathcal{L}^{2}(T)} & \leqslant C h\|\mathbf{v}-\mathbf{R}\|_{\mathcal{L}^{\infty}(T)} \\
& \leqslant C h\left(\left\|\mathbf{v}-\mathbf{v}_{I h}\right\|_{\mathcal{L}^{\infty}(T)}+\left\|\mathbf{v}_{I h}-\mathbf{R}\right\|_{\mathcal{L}^{\infty}(T)}\right)
\end{aligned}
$$


By using a classical interpolation error (see, for example, [2, Corollary 4.4.7]) and an inverse estimate (see, for example, [2, Lemma 4.5.3]), the above inequality yields

$$
\left\|\mathbf{v}_{I h}-\mathbf{v}_{h}\right\|_{\mathcal{L}^{2}(T)} \leqslant C h\left(h\|\mathbf{v}\|_{\mathcal{H}^{2}(T)}+h^{-1}\left\|\mathbf{v}_{I h}-\mathbf{R}\right\|_{\mathcal{L}^{2}(T)}\right)
$$

which implies that

$$
\begin{aligned}
\left\|\mathbf{v}_{I h}-\mathbf{v}_{h}\right\|_{\mathcal{L}^{2}(T)} & \leqslant C\left(h^{2}\|\mathbf{v}\|_{\mathcal{H}^{2}(T)}+\left\|\mathbf{v}_{I h}-\mathbf{v}\right\|_{\mathcal{L}^{2}(T)}+\|\mathbf{v}-\mathbf{R}\|_{\mathcal{L}^{2}(T)}\right) \\
& \leqslant C\left(h^{2}\|\mathbf{v}\|_{\mathcal{H}^{2}(T)}+\|\mathbf{v}-\mathbf{R}\|_{\mathcal{L}^{2}(T)}\right) .
\end{aligned}
$$

Above we have used again a classical result on the interpolation error (see, for example, [2, Theorem 4.4.4]).

Now, summing up the above relation for all triangles $T \in \mathcal{F}_{3}$ we obtain that

$$
\left\|\mathbf{v}_{I h}-\mathbf{v}_{h}\right\|_{\mathcal{L}^{2}\left(\cup_{T \in \mathcal{F}_{3}} T\right)} \leqslant C\left(h^{2}\|\mathbf{v}\|_{\mathcal{H}^{2}(\mathcal{O} \backslash B(\boldsymbol{\zeta}))}+\|\mathbf{v}-\mathbf{R}\|_{\mathcal{L}^{2}\left(\cup_{T \in \mathcal{F}_{3}} T\right)}\right) .
$$

In order to estimate the last term in the right-hand side of (4.18) we proceed as previously by introducing the closed ball $B_{2 h}(\boldsymbol{\zeta})$ of center $\boldsymbol{\zeta}$ and of radius $1+2 h$. This ball is included in $\mathcal{O}$ thanks to (4.1). It is clear that all triangles of $\mathcal{F}_{3}$ are contained in $B_{2 h}(\boldsymbol{\zeta}) \backslash B(\boldsymbol{\zeta})$. Then, we can use once again Lemma 5.11 in Fujita and Sauer [10] and prove an estimate similar to (4.9) namely

$$
\|\mathbf{v}-\mathbf{R}\|_{\mathcal{L}^{2}\left(B_{2 h}(\boldsymbol{\zeta}) \backslash B(\boldsymbol{\zeta})\right)} \leqslant C h^{\frac{3}{2}}\|\mathbf{v}-\mathbf{R}\|_{\mathcal{H}^{2}(\mathcal{O} \backslash B(\boldsymbol{\zeta}))} .
$$

From (4.18) and (4.19) we deduce that

$$
\left\|\mathbf{v}_{I h}-\mathbf{v}_{h}\right\|_{\mathcal{L}^{2}\left(\cup_{T \in \mathcal{F}_{3}} T\right)} \leqslant C h^{\frac{3}{2}}\left(\|\mathbf{v}\|_{\mathcal{H}^{2}(\mathcal{O} \backslash B(\boldsymbol{\zeta}))}+\|\mathbf{v}\|_{\mathcal{H}^{2}(B(\boldsymbol{\zeta}))}\right) .
$$

The above relation, combined to (4.13) and to interpolation error estimate (see [2, Theorem 4.4.4]) implies that

$$
\left\|\mathbf{v}-\mathbf{v}_{h}\right\|_{\mathcal{L}^{2}\left(\cup_{T \in \mathcal{F}_{3}} T\right)} \leqslant C h^{\frac{3}{2}}\left(\|\mathbf{v}\|_{\mathcal{H}^{2}(\mathcal{O} \backslash B(\boldsymbol{\zeta}))}+\|\mathbf{v}\|_{\mathcal{H}^{2}(B(\boldsymbol{\zeta}))}\right) .
$$

Now we turn to the $H^{1}$-estimate for the family $\mathcal{F}_{3}$ of triangles. From the usual inverse inequality (see [2, Lemma 4.5.3]) and the $L^{2}$-estimate (4.20) we obtain

$$
\left\|\nabla\left(\mathbf{v}_{I h}-\mathbf{v}_{h}\right)\right\|_{\left[L^{2}\left(\cup_{T \in \mathcal{F}_{3}} T\right)\right]^{4}} \leq C_{1} h^{\frac{1}{2}}\left(\|\mathbf{v}\|_{\mathcal{H}^{2}(\mathcal{O} \backslash B(\boldsymbol{\zeta}))}+\|\mathbf{v}\|_{\mathcal{H}^{2}(B(\boldsymbol{\zeta}))}\right),
$$

which implies together with (4.13) and with interpolation error estimate (see [2, Theorem 4.4.4]) that

$$
\left\|\nabla\left(\mathbf{v}-\mathbf{v}_{h}\right)\right\|_{\left[L^{2}\left(\bigcup_{T \in \mathcal{F}_{3}} T\right)\right]^{4}} \leq C_{1} h^{\frac{1}{2}}\left(\|\mathbf{v}\|_{\mathcal{H}^{2}(\mathcal{O} \backslash B(\boldsymbol{\zeta}))}+\|\mathbf{v}\|_{\mathcal{H}^{2}(B(\boldsymbol{\zeta}))}\right)
$$

Finally, we consider the case of the triangles family $\mathcal{F}_{4}$. Interpolation error estimates lead to

$$
\left\|\mathbf{v}-\mathbf{v}_{h}\right\|_{L^{2}\left(\bigcup_{T \in \mathcal{F}_{4}} T\right)} \leq C_{1} h^{2}\|\mathbf{v}\|_{\mathcal{H}^{2}(\mathcal{O} \backslash B(\boldsymbol{\zeta}))}
$$


and

$$
\left\|\nabla\left(\mathbf{v}-\mathbf{v}_{h}\right)\right\|_{L^{2}\left(\cup_{T \in \mathcal{F}_{4}} T\right)} \leq C_{1} h\|\mathbf{v}\|_{\mathcal{H}^{2}(\mathcal{O} \backslash B(\boldsymbol{\zeta}))} .
$$

Relations (4.11), (4.21), (4.24) and the fact that $\mathbf{v}=\mathbf{v}_{h}$ in $B(\boldsymbol{\zeta})$ imply (4.3). Moreover, (4.12), (4.23), (4.25) and the fact that $\mathbf{v}=\mathbf{v}_{h}$ in $B(\boldsymbol{\zeta})$ imply (4.4).

Lemma 4.2. There exists a positive constant $C_{2}$ (not depending of the position of $B(\boldsymbol{\zeta})$ ) such that

$$
\inf _{q_{h} \in M_{h}(\boldsymbol{\zeta})}\left\|q-q_{h}\right\|_{L^{2}(\mathcal{O})} \leqslant C_{2} h^{\frac{1}{2}}\|q\|_{H^{1}(\mathcal{O} \backslash B(\boldsymbol{\zeta}))},
$$

for all $q \in M(\boldsymbol{\zeta}) \cap H^{1}(\mathcal{O} \backslash B(\boldsymbol{\zeta}))$.

Proof. The proof of this lemma is similar to the one of Lemma 4.1. Consider a function $q \in M(\boldsymbol{\zeta}) \cap H^{1}(\mathcal{O} \backslash B(\boldsymbol{\zeta}))$. According to a classical result (see, for example, [3, Theorem IX.7]), there exists $\widetilde{q} \in H^{1}(\mathcal{O})$ such that

$$
\widetilde{q}_{\mid \mathcal{O} \backslash B(\boldsymbol{\zeta})}=q, \quad\|\widetilde{q}\|_{H^{1}(\mathcal{O})} \leq C\|q\|_{H^{1}(\mathcal{O} \backslash B(\boldsymbol{\zeta}))},
$$

and it can be proved that we can choose the constant $C$ independent of the position of $B(\boldsymbol{\zeta})$. Moreover, by a classical interpolation argument (see, for example, [2, Theorem 4.4.4]), there exists $\widetilde{q}_{h} \in E_{h}$ such that

$$
\left\|\widetilde{q}-\widetilde{q}_{h}\right\|_{L^{2}(\mathcal{O})} \leq C h\|\widetilde{q}\|_{H^{1}(\mathcal{O})} .
$$

The above relation and (4.27) clearly imply that there exists a constant $C>0$ such that

$$
\left\|q-\widetilde{q}_{h}\right\|_{L^{2}(\mathcal{O} \backslash B(\boldsymbol{\zeta}))} \leq C h\|q\|_{H^{1}(\mathcal{O} \backslash B(\zeta))} .
$$

Denote by $q_{h}$ the function in $E_{h}$ satisfying the conditions

$$
\begin{gathered}
q_{h}\left(\mathbf{x}_{i}\right)=0 \quad \text { if } \mathbf{x}_{i} \in \overline{A_{h}}, \\
q_{h}\left(\mathbf{x}_{i}\right)=\widetilde{q}_{h}\left(\mathbf{x}_{i}\right) \text { if } \mathbf{x}_{i} \in \mathcal{T}_{h} \backslash \overline{A_{h}} .
\end{gathered}
$$

Then as in the proof of Lemma 4.1, we can show that

$$
\left\|q-q_{h}\right\|_{L^{2}(\mathcal{O})} \leqslant C_{2} h^{\frac{1}{2}}\|q\|_{H^{1}(\mathcal{O} \backslash B(\boldsymbol{\zeta}))} .
$$

We next show that the finite element spaces $\mathcal{K}_{h}(\boldsymbol{\zeta}), M_{h}(\boldsymbol{\zeta})$ and the bilinear form $b$ satisfy a discrete inf-sup condition. This proves in particular that the approximate problem (3.5)(3.6) is well-posed (see [12, Theorem II.1.1., pp.114]). More precisely, the following result holds.

Lemma 4.3. There exists a constant $\beta^{*}>0$ such that, for all $q_{h} \in M_{h}(\boldsymbol{\zeta})$ there exists $\mathbf{u}_{h} \in \mathcal{K}_{h}(\boldsymbol{\zeta})$ with

$$
\int_{\mathcal{O}} \operatorname{div}\left(\mathbf{u}_{h}\right) q_{h} \mathrm{~d} \mathbf{x} \geqslant \beta^{*}\left\|\mathbf{u}_{h}\right\|_{\mathcal{H}^{1}(\mathcal{O})}\left\|q_{h}\right\|_{L^{2}(\mathcal{O})}
$$


Proof. Let $q_{h} \in M_{h}(\boldsymbol{\zeta})$. Since $M_{h}(\boldsymbol{\zeta}) \subset M(\boldsymbol{\zeta})$, Lemma 2.6 yields the existence of $\mathbf{u} \in \mathcal{K}(\boldsymbol{\zeta})$ such that

$$
\int_{\mathcal{O}} \operatorname{div}(\mathbf{u}) q_{h} \mathrm{~d} \mathbf{x} \geqslant \beta\|\mathbf{u}\|_{\mathcal{H}^{1}(\mathcal{O})}\left\|q_{h}\right\|_{L^{2}(\mathcal{O})},
$$

with $\beta$ not depending on $q_{h}$. In order to prove the conclusion of the lemma it suffices to show the existence of $\mathbf{u}_{h} \in \mathcal{K}_{h}(\boldsymbol{\zeta})$ such that

$$
\begin{gathered}
\int_{\mathcal{O}} \operatorname{div}\left(\mathbf{u}_{h}\right) q_{h} \mathrm{~d} \mathbf{x}=\int_{\mathcal{O}} \operatorname{div}(\mathbf{u}) q_{h} \mathrm{~d} \mathbf{x}, \\
\left\|\mathbf{u}_{h}\right\|_{\mathcal{H}^{1}(\mathcal{O})} \leqslant C\|\mathbf{u}\|_{\mathcal{H}^{1}(\mathcal{O})},
\end{gathered}
$$

where $C$ is a constant independent of $q_{h}$.

Note that (4.30) is equivalent to

$$
\int_{\mathcal{O}} \mathbf{u}_{h} \cdot \nabla q_{h} \mathrm{~d} \mathbf{x}=\int_{\mathcal{O}} \mathbf{u} \cdot \nabla q_{h} \mathrm{~d} \mathbf{x}
$$

Since $\nabla q_{h}$ is constant in each triangle and it vanishes in any triangle from $\mathcal{F}_{1} \cup \mathcal{F}_{2}$, in order to check (4.30), it suffices to show that

$$
\int_{T} \mathbf{u}_{h} \mathrm{~d} \mathbf{x}=\int_{T} \mathbf{u} \mathrm{d} \mathbf{x} \quad \forall T \in \mathcal{F}_{3} \cup \mathcal{F}_{4} .
$$

Notice first that if $\mathbf{u}_{h} \in \mathcal{K}_{h}(\boldsymbol{\zeta})$ then, for any triangle $T \in \mathcal{T}_{h}$ of vertices $\mathbf{x}_{1}, \mathbf{x}_{2}, \mathbf{x}_{3}$ and of corresponding barycentric functions $\varphi_{1}, \varphi_{2}, \varphi_{3}$, we have

$$
\mathbf{u}_{h}(\mathbf{x})=\overline{\mathbf{u}}_{h}(x)+\frac{\varphi_{1}(\mathbf{x}) \varphi_{2}(\mathbf{x}) \varphi_{3}(\mathbf{x})}{\int_{T} \varphi_{1} \varphi_{2} \varphi_{3} \mathrm{~d} \mathbf{x}} \boldsymbol{\lambda} \quad \forall \mathbf{x} \in T
$$

where $\overline{\mathbf{u}}_{h} \in \mathcal{C}(\overline{\mathcal{O}})$ satisfies

$$
\overline{\mathbf{u}}_{h}(x)=\varphi_{1}(\mathbf{x}) \boldsymbol{\alpha}_{\mathbf{1}}+\varphi_{2}(\mathbf{x}) \boldsymbol{\alpha}_{\mathbf{2}}+\varphi_{3}(\mathbf{x}) \boldsymbol{\alpha}_{\mathbf{3}} \quad \forall \mathbf{x} \in T,
$$

for some constant vectors $\boldsymbol{\alpha}_{\mathbf{1}}, \boldsymbol{\alpha}_{\mathbf{2}}, \boldsymbol{\alpha}_{\mathbf{3}}, \boldsymbol{\lambda} \in \mathbb{R}^{2}$ (these constants depend on the triangle $T$ ). Notice that, since the restriction of $\mathbf{u}_{h}$ to triangles in $\mathcal{F}_{1} \cup \mathcal{F}_{2}$ is a rigid velocity field, the constant $\boldsymbol{\lambda}$ in (4.33) is equal to zero, for all triangles in $\mathcal{F}_{1} \cup \mathcal{F}_{2}$. If $\overline{\mathbf{u}}_{h}$ satisfies (4.34) and $T \in \mathcal{F}_{3} \cup \mathcal{F}_{4}$ then condition (4.32) holds provided that

$$
\lambda=\int_{T}\left(\mathbf{u}-\overline{\mathbf{u}}_{h}\right) \mathrm{d} \mathbf{x} \quad \forall T \in \mathcal{F}_{3} \cup \mathcal{F}_{4}
$$

Some simple calculations show that there exists a constant $C>0$ (not depending on the triangle) such that

$$
\left\|\frac{\varphi_{1} \varphi_{2} \varphi_{3}}{\int_{T} \varphi_{1} \varphi_{2} \varphi_{3} \mathrm{~d} \mathbf{x}}\right\|_{H^{1}(T)} \leqslant \frac{C}{h^{2}} .
$$


Moreover, (4.35) and the Cauchy-Schwarz inequality imply that

$$
|\lambda| \leqslant C h\left\|\mathbf{u}-\overline{\mathbf{u}}_{h}\right\|_{\mathcal{L}^{2}(T)} \quad \forall T \in \mathcal{F}_{3} \cup \mathcal{F}_{4},
$$

for some constant $C$. From (4.33), (4.36) and (4.37) it follows that

$$
\left\|\mathbf{u}_{h}\right\|_{\mathcal{H}^{1}(T)} \leqslant\left\|\overline{\mathbf{u}}_{h}\right\|_{\mathcal{H}^{1}(T)}+\frac{C}{h}\left\|\mathbf{u}-\overline{\mathbf{u}}_{h}\right\|_{\mathcal{L}^{2}(T)} \quad \forall T \in \mathcal{F}_{3} \cup \mathcal{F}_{4}
$$

The remaining part of the proof is devoted to the construction of $\overline{\mathbf{u}}_{h}$ such that $\mathbf{u}_{h}$ satisfies (4.31). According to a classical result (see, for instance, [12, Theorem I.A.2., pp.101]), there exists a function $\overline{\mathbf{u}}_{h}^{c} \in C(\overline{\mathcal{O}})$ which is affine in each triangle $T \in \mathcal{T}_{h}$ such that

$$
\begin{gathered}
\left\|\mathbf{u}-\overline{\mathbf{u}}_{h}^{c}\right\|_{\mathcal{L}^{2}(T)} \leq C h\|\mathbf{u}\|_{\mathcal{H}^{1}(T)}, \\
\left\|\overline{\mathbf{u}}_{h}^{c}\right\|_{\mathcal{H}^{1}(T)} \leq C\|\mathbf{u}\|_{\mathcal{H}^{1}(T)},
\end{gathered}
$$

with the constant $C$ not depending on $h$. We are now in a position to define $\overline{\mathbf{u}}_{h}$. This function is defined by

$$
\overline{\mathbf{u}_{h}}(\mathbf{x})=\left\{\begin{array}{lll}
\overline{\mathbf{u}}_{h}^{c}(\mathbf{x}) & \text { if } & \mathbf{x} \in \bigcup_{T \in \mathcal{F}_{4}} T \\
\mathbf{R}(\mathbf{x}) & \text { if } & \mathbf{x} \in \bigcup_{T \in \mathcal{F}_{1} \cup \mathcal{F}_{2}} T
\end{array}\right.
$$

where $\mathbf{R}$ is the extension of $\mathbf{u}_{\mid B(\boldsymbol{\zeta})}$ (which is a rigid velocity field) to $\mathbb{R}^{2}$. We remark that relation (4.41) also defines the values of $\overline{\mathbf{u}}_{h}$ in the triangles of $\mathcal{F}_{3}$. Indeed, the vertices of each triangle in $\mathcal{F}_{3}$ are also vertices of a triangle in either $\mathcal{F}_{2}$ or in $\mathcal{F}_{4}$. In order to prove (4.31) we estimate the terms in the right hand side of (4.38). We first consider a triangle $T \in \mathcal{F}_{4}$. By using the fact that $\overline{\mathbf{u}}_{h}=\overline{\mathbf{u}}_{h}^{c}$ in $T$, (4.39) and (4.40) we obtain that

$$
\left\|\overline{\mathbf{u}}_{h}\right\|_{\mathcal{H}^{1}(T)}+\frac{1}{h}\left\|\mathbf{u}-\overline{\mathbf{u}}_{h}\right\|_{\mathcal{L}^{2}(T)} \leq C\|\mathbf{u}\|_{\mathcal{H}^{1}(T)} \quad \forall T \in \mathcal{F}_{4}
$$

with the constant $C$ not depending on $\mathbf{u}$. We next consider a triangle $T \in \mathcal{F}_{3}$. We first notice that

$$
\begin{aligned}
&\left\|\overline{\mathbf{u}}_{h}\right\|_{\mathcal{H}^{1}(T)}+\frac{1}{h}\left\|\mathbf{u}-\overline{\mathbf{u}}_{h}\right\|_{\mathcal{L}^{2}(T)} \leq\left\|\overline{\mathbf{u}}_{h}^{c}\right\|_{\mathcal{H}^{1}(T)}+\frac{1}{h}\left\|\mathbf{u}-\overline{\mathbf{u}}_{h}^{c}\right\|_{\mathcal{L}^{2}(T)} \\
&+\left\|\overline{\mathbf{u}}_{h}^{c}-\overline{\mathbf{u}}_{h}\right\|_{\mathcal{H}^{1}(T)}+\frac{1}{h}\left\|\overline{\mathbf{u}}_{h}-\overline{\mathbf{u}}_{h}^{c}\right\|_{\mathcal{L}^{2}(T)} \quad \forall T \in \mathcal{F}_{3} .
\end{aligned}
$$

The first two terms in the right hand side of (4.43) can be directly estimated by using (4.39) and (4.40). Moreover, by using inverse estimates (see, for example, [2, Lemma 4.5.3]), there exists a positive constant $C$ not depending on $h$ such that

$$
\left\|\overline{\mathbf{u}}_{h}^{c}-\overline{\mathbf{u}}_{h}\right\|_{\mathcal{H}^{1}(T)}+\frac{1}{h}\left\|\overline{\mathbf{u}}_{h}-\overline{\mathbf{u}}_{h}^{c}\right\|_{\mathcal{L}^{2}(T)} \leqslant C\left\|\overline{\mathbf{u}}_{h}^{c}-\overline{\mathbf{u}}_{h}\right\|_{\mathcal{L}^{\infty}(T)} \quad \forall T \in \mathcal{F}_{3} .
$$


The above relation and the fact that $\overline{\mathbf{u}}_{h}$ is equal either to $\mathbf{R}$ or to $\overline{\mathbf{u}}_{h}^{c}$ in the vertices of a triangle $T \in \mathcal{F}_{3}$ imply that

$$
\left\|\overline{\mathbf{u}}_{h}^{c}-\overline{\mathbf{u}}_{h}\right\|_{\mathcal{H}^{1}(T)}+\frac{1}{h}\left\|\mathbf{u}_{h}-\overline{\mathbf{u}}_{h}^{c}\right\|_{\mathcal{L}^{2}(T)} \leq C\left\|\overline{\mathbf{u}}_{h}^{c}-\mathbf{R}\right\|_{\mathcal{L}^{\infty}(T)} \quad \forall T \in \mathcal{F}_{3} .
$$

The above inequality, combined once again, with an inverse inequality implies that

$$
\left\|\overline{\mathbf{u}}_{h}^{c}-\overline{\mathbf{u}}_{h}\right\|_{\mathcal{H}^{1}(T)}+\frac{1}{h}\left\|\mathbf{u}_{h}-\overline{\mathbf{u}}_{h}^{c}\right\|_{\mathcal{L}^{2}(T)} \leq \frac{C}{h}\left\|\overline{\mathbf{u}}_{h}^{c}-\mathbf{R}\right\|_{\mathcal{L}^{2}(T)} \quad \forall T \in \mathcal{F}_{3}
$$

On the other hand

$$
\left\|\overline{\mathbf{u}}_{h}^{c}-\mathbf{R}\right\|_{\mathcal{L}^{2}(T)} \leq\left\|\overline{\mathbf{u}}_{h}^{c}-\mathbf{u}\right\|_{\mathcal{L}^{2}(T)}+\|\mathbf{u}-\mathbf{R}\|_{\mathcal{L}^{2}(T)} \quad \forall T \in \mathcal{F}_{3}
$$

Combining (4.39), (4.45), (4.44) and (4.43) we obtain

$$
\left\|\overline{\mathbf{u}}_{h}\right\|_{\mathcal{H}^{1}(T)}+\frac{1}{h}\left\|\mathbf{u}-\overline{\mathbf{u}}_{h}\right\|_{\mathcal{L}^{2}(T)} \leq C\|\mathbf{u}\|_{\mathcal{H}^{1}(T)}+\frac{C}{h}\|\mathbf{u}-\mathbf{R}\|_{\mathcal{L}^{2}(T)} \quad \forall T \in \mathcal{F}_{3}
$$

We recall that all triangles of $\mathcal{F}_{3}$ are contained in $B_{2 h}(\boldsymbol{\zeta}) \backslash B(\boldsymbol{\zeta})$. Therefore, by taking the sum of the above relation for all $T \in \mathcal{F}_{3}$ and by using (4.6), combined to the fact that $\mathbf{u}=\mathbf{R}$ on $\partial B(\boldsymbol{\zeta})$, we obtain

$$
\left.\left.\left\|\overline{\mathbf{u}}_{h}\right\|_{\mathcal{H}^{1}} \underset{T \in \mathcal{F}_{3}}{\bigcup T}\right)+\frac{1}{h}\left\|\mathbf{u}-\overline{\mathbf{u}}_{h}\right\|_{\mathcal{L}^{2}} \underset{T \in \mathcal{F}_{3}}{\cup} T\right) \leq C\|\mathbf{u}\|_{\mathcal{H}^{1}\left(B_{2 h}(\boldsymbol{\zeta}) \backslash B(\boldsymbol{\zeta})\right)} .
$$

Now by combining (4.42) and (4.47) in (4.38) we obtain

$$
\left.\left\|\mathbf{u}_{h}\right\|_{\mathcal{H}^{1}} \underset{T \in \mathcal{F}_{3} \cup \mathcal{F}_{4}}{\cup} T\right) \leq C\|\mathbf{u}\|_{\mathcal{H}^{1}(\mathcal{O} \backslash B(\boldsymbol{\zeta}))} .
$$

We next consider the triangles $T \in \mathcal{F}_{1} \cup \mathcal{F}_{2}$. By using the fact that $\mathbf{u}_{h}=\overline{\mathbf{u}}_{h}=\mathbf{R}$ in $T$ we obtain that

$$
\left.\left.\left\|\mathbf{u}_{h}\right\|_{\mathcal{H}^{1}} \underset{T \in \mathcal{F}_{1} \cup \mathcal{F}_{2}}{\cup} T\right)=\|\mathbf{R}\|_{\mathcal{H}^{1}} \underset{T \in \mathcal{F}_{1} \cup \mathcal{F}_{2}}{\bigcup} T\right) .
$$

A simple calculation shows that the right hand side of the above relation is bounded by $C\|\mathbf{u}\|_{\mathcal{H}^{1}(B(\boldsymbol{\zeta}))}$, where $C$ is a constant not depending on $h$. We thus obtain

$$
\left.\left\|\mathbf{u}_{h}\right\|_{\mathcal{H}^{1}} \underset{T \in \mathcal{F}_{1} \cup \mathcal{F}_{2}}{\cup} T\right) \leq C\|\mathbf{u}\|_{\mathcal{H}^{1}(B(\boldsymbol{\zeta}))} .
$$

If we join (4.48) and (4.49) we see that the function $\mathbf{u}_{h}$ satisfies (4.31). This ends up the proof of the Lemma.

Now, we are in position to introduce a projector in $\mathcal{K}_{h}(\boldsymbol{\zeta}) \times M_{h}(\boldsymbol{\zeta})$ that will be a key ingredient in the proof of the convergence result. 
Lemma 4.4. Suppose that $\mathbf{V} \in \mathcal{K}(\boldsymbol{\zeta})$ and that $P \in M(\boldsymbol{\zeta})$. Then there exists a unique couple $\left(\mathbf{V}_{h}, P_{h}\right)$ in $\mathcal{K}_{h}(\boldsymbol{\zeta}) \times M_{h}(\boldsymbol{\zeta})$ such that:

$$
\left\{\begin{aligned}
a\left(\mathbf{V}-\mathbf{V}_{h}, \boldsymbol{\varphi}\right)+b\left(\boldsymbol{\varphi}, P-P_{h}\right) & =0 & & \forall \boldsymbol{\varphi} \in \mathcal{K}_{h}(\boldsymbol{\zeta}) \\
b\left(\mathbf{V}-\mathbf{V}_{h}, q\right) & =0 & & \forall q \in M_{h}(\boldsymbol{\zeta}) .
\end{aligned}\right.
$$

Moreover, if we suppose in addition that $\mathbf{V}_{\mid \mathcal{O} \backslash B(\boldsymbol{\zeta})} \in \mathcal{H}^{2}(\mathcal{O} \backslash B(\boldsymbol{\zeta}))$ and that $P_{\mid \mathcal{O} \backslash B(\boldsymbol{\zeta})} \in$ $H^{1}(\mathcal{O} \backslash B(\boldsymbol{\zeta}))$ then there exists a positive constant $C$ such that

$$
\left\|\mathbf{V}-\mathbf{V}_{h}\right\|_{\mathcal{L}^{2}(\mathcal{O})} \leqslant C h .
$$

Proof. The result in Lemma 4.3 combined to Theorem 1.1 in [12, p.114] implies the existence and uniqueness of $\left(\mathbf{V}_{h}, P_{h}\right)$ in $\mathcal{K}_{h}(\boldsymbol{\zeta}) \times M_{h}(\boldsymbol{\zeta})$ satisfying (4.50) together with

$$
\left\|\mathbf{V}-\mathbf{V}_{h}\right\|_{\mathcal{H}^{1}(\mathcal{O})}+\left\|P-P_{h}\right\|_{L^{2}(\mathcal{O})} \leqslant C\left\{\inf _{\mathbf{v} \in \mathcal{K}_{h}(\boldsymbol{\zeta})}\|\mathbf{V}-\mathbf{v}\|_{\mathcal{H}^{1}(\mathcal{O})}+\inf _{q \in M_{h}(\boldsymbol{\zeta})}\|P-q\|_{L^{2}(\mathcal{O})}\right\}
$$

Using Lemmas 4.1 and 4.2 we obtain

$$
\left\|\mathbf{V}-\mathbf{V}_{h}\right\|_{\mathcal{H}^{1}(\mathcal{O})}+\left\|P-P_{h}\right\|_{L^{2}(\mathcal{O})} \leqslant C h^{1 / 2}\left\{\|\mathbf{V}\|_{\mathcal{H}^{2}(\mathcal{O} \backslash B)}+\|\mathbf{V}\|_{\mathcal{H}^{2}(B)}+\|P\|_{H^{1}(\mathcal{O})}\right\} .
$$

Moreover, by applying the usual Aubin-Nitsche duality argument (see for example [12, pp.119]), one can easily prove

$$
\left\|\mathbf{V}-\mathbf{V}_{h}\right\|_{\mathcal{L}^{2}(\mathcal{O})} \leqslant C h\left\{\|\mathbf{V}\|_{\mathcal{H}^{2}(\mathcal{O} \backslash B)}+\|\mathbf{V}\|_{\mathcal{H}^{2}(B)}+\|P\|_{H^{1}(\mathcal{O})}\right\}
$$

\section{Definition and properties of the change of vari- ables.}

In order to prove Theorem 3.2, we should be able to compare the exact solution which is rigid in the ball $B\left(\boldsymbol{\zeta}\left(t_{k}\right)\right)$ with the approximate solution which is rigid in the ball $B\left(\boldsymbol{\zeta}_{h}^{k}\right)$. This will be achieved by the use of a change of variables which maps the exact ball onto the approximate one. This section is devoted to the description and main properties of this transformation.

\subsection{Change of variables.}

In this section, we suppose that $\mathcal{O}$ is convex. In the sequel, we need a change of variables transforming a function in $\widehat{\mathcal{K}}\left(\boldsymbol{\zeta}_{1}\right)$ into a function in $\widehat{\mathcal{K}}\left(\boldsymbol{\zeta}_{2}\right)$, where $\boldsymbol{\zeta}_{i} \in \mathcal{O}$ are such that

$$
\operatorname{dist}\left(\boldsymbol{\zeta}_{i}, \partial \mathcal{O}\right)>1+2 \eta, \quad i \in\{1,2\} \text {, with } \eta>0 \text {. }
$$

In this case, $B\left(\boldsymbol{\zeta}_{i}\right)$ is contained in $\mathcal{O}$ and the distance between $B\left(\boldsymbol{\zeta}_{i}\right)$ and $\partial \mathcal{O}$ is greater than $2 \eta$. Let $\xi \in C^{\infty}\left(\mathbb{R}^{2}, \mathbb{R}\right)$ be a compactly supported function such that 
- $\xi=1$ if $\mathbf{x} \in \mathcal{O}$ and $\operatorname{dist}(\mathbf{x}, \partial \mathcal{O})>2 \eta$

- $\xi=0$ if $\mathbf{x} \notin \mathcal{O}$ or $\operatorname{dist}(\mathbf{x}, \partial \mathcal{O}) \leqslant \eta$

Let $\Lambda$ be the mapping defined by

$$
\boldsymbol{\Lambda}(\mathbf{x})=\left[\left(\boldsymbol{\zeta}_{1}-\boldsymbol{\zeta}_{2}\right) \cdot \mathbf{x}^{\perp}\right](\operatorname{rot} \xi)+\xi\left(\boldsymbol{\zeta}_{1}-\boldsymbol{\zeta}_{2}\right) \quad \forall \mathbf{x} \in \mathbb{R}^{2} .
$$

We need several properties of the field $\Lambda$ and of the associated flow. Since these properties are similar to those proved in [27] we state them here without proof.

Lemma 5.1. Let $\boldsymbol{\Lambda}$ be the mapping defined by (5.2). Then we have

(i) $\boldsymbol{\Lambda}=0$ outside $\mathcal{O}$,

(ii) $\operatorname{div} \boldsymbol{\Lambda}=0$ in $\mathbb{R}^{2}$,

(iii) $\boldsymbol{\Lambda}(\mathbf{x})=\boldsymbol{\zeta}_{1}-\boldsymbol{\zeta}_{2}$ if $\mathbf{x} \in \mathcal{O}$ and if $\operatorname{dist}(\mathbf{x}, \partial \mathcal{O})>2 \eta$.

In other words, the restriction of $\boldsymbol{\Lambda}$ to a neighbourhood of $\partial \mathcal{O}$ is zero and $\boldsymbol{\Lambda}$ is a translation when restricted to points of $\mathcal{O}$ at distance to $\partial \mathcal{O}$ larger than $2 \eta$.

We consider next the initial value problem:

$$
\left\{\begin{array}{l}
\frac{d}{d \lambda} \boldsymbol{\psi}(\lambda)=\boldsymbol{\Lambda}(\boldsymbol{\psi}(\lambda)), \quad \lambda>0 \\
\boldsymbol{\psi}(0)=\mathbf{y}
\end{array}\right.
$$

with $\boldsymbol{\Lambda}$ given by (5.2).

Lemma 5.2. For all $\mathbf{y} \in \mathbb{R}^{2}$, the initial value problem (5.3) admits a unique solution $\boldsymbol{\psi}(\lambda, \mathbf{y})$ on $[0,1]$. Denote

$$
\mathbf{X}_{\boldsymbol{\zeta}_{2}, \boldsymbol{\zeta}_{1}}(\mathbf{y})=\mathbf{X}(\mathbf{y})=\boldsymbol{\psi}(1, \mathbf{y})
$$

Then $\mathbf{X}$ is a $C^{\infty}$-diffeomorphism from $\mathcal{O}$ onto itself and $\mathbf{X}\left(B\left(\boldsymbol{\zeta}_{2}\right)\right)=B\left(\boldsymbol{\zeta}_{1}\right)$. If we denote by

$$
\mathbf{J}_{\mathbf{X}}=\left(\frac{\partial X_{i}}{\partial y_{j}}\right)_{i, j}
$$

the jacobian matrix of the transformation $\mathbf{y} \mapsto \mathbf{X}(\mathbf{y})$, then the above change of variables satisfies:

$$
\operatorname{det} \mathbf{J}_{\mathbf{X}}(\mathbf{y})=1 \quad \forall \mathbf{y} \in \mathbb{R}^{2} .
$$

We denote by

$$
\mathbf{Y}_{\boldsymbol{\zeta}_{2}, \boldsymbol{\zeta}_{1}}=\mathbf{Y}=\mathbf{X}^{-1}
$$

the inverse of $\mathbf{X}$ on $\mathcal{O}$. 


\subsection{Properties of the change of variables.}

In this subsection, we use the change of variables defined by the mapping $\mathbf{X}$ in Lemma 5.2 to transform functions in $\widehat{\mathcal{K}}\left(\boldsymbol{\zeta}_{1}\right)$ (resp. $\mathcal{K}\left(\boldsymbol{\zeta}_{1}\right), M\left(\boldsymbol{\zeta}_{1}\right)$ ) into functions in $\widehat{\mathcal{K}}\left(\boldsymbol{\zeta}_{2}\right)$ (resp. $\left.\mathcal{K}\left(\boldsymbol{\zeta}_{2}\right), M\left(\boldsymbol{\zeta}_{2}\right)\right)$. We also give the expressions of $\Delta \mathbf{u}$ and of $\nabla p$ after the transformation.

Consider $(\mathbf{u}, p) \in \mathcal{H}^{1}(\mathcal{O}) \times L^{2}(\mathcal{O})$ and define as in [18] the functions $(\mathbf{U}, P) \in \mathcal{H}^{1}(\mathcal{O}) \times$ $L^{2}(\mathcal{O})$ by

$$
\begin{gathered}
\mathbf{U}(\mathbf{y})=\mathbf{J}_{\mathbf{Y}}(\mathbf{X}(\mathbf{y})) \mathbf{u}(\mathbf{X}(\mathbf{y})) \quad \forall \mathbf{y} \in \mathcal{O}, \\
P(\mathbf{y})=p(\mathbf{X}(\mathbf{y})) \quad \forall \mathbf{y} \in \mathcal{O} .
\end{gathered}
$$

We can easily check, by using the definition of $\boldsymbol{\Lambda}$ that

$$
\begin{array}{ll}
\mathbf{X}(\mathbf{y})=\mathbf{y}+\boldsymbol{\zeta}_{1}-\boldsymbol{\zeta}_{2} & \forall \mathbf{y} \in B\left(\boldsymbol{\zeta}_{2}\right), \\
\mathbf{Y}(\mathbf{x})=\mathbf{x}-\boldsymbol{\zeta}_{1}+\boldsymbol{\zeta}_{2} & \forall \mathbf{x} \in B\left(\boldsymbol{\zeta}_{1}\right),
\end{array}
$$

Consequently, if $\mathbf{u} \in \mathcal{K}\left(\boldsymbol{\zeta}_{1}\right)$ then $\mathbf{U} \in \mathcal{K}\left(\boldsymbol{\zeta}_{2}\right)$ and that if $p \in M\left(\boldsymbol{\zeta}_{1}\right)$ then $P \in M\left(\boldsymbol{\zeta}_{2}\right)$.

By using (5.5), we obtain the following result (see, for instance, [18, Proposition 2.4]).

Lemma 5.3. If $\mathbf{X}$ is defined by (5.4), then for all $\mathbf{u} \in \mathcal{H}^{1}(\mathcal{O})$, the function $\mathbf{U}$ defined as above satisfies the following relation:

$$
\operatorname{div}[\mathbf{U}(\mathbf{y})]=\operatorname{div}[\mathbf{u}(\mathbf{X}(\mathbf{y}))] \quad \forall \mathbf{y} \in \mathcal{O} .
$$

This lemma implies in particular that if $\mathbf{u} \in \widehat{\mathcal{K}}\left(\boldsymbol{\zeta}_{1}\right)$ then $\mathbf{U} \in \widehat{\mathcal{K}}\left(\boldsymbol{\zeta}_{2}\right)$.

In order to write down the expressions of $\Delta \mathbf{u}$ and $\nabla p$ after change of variables, we define (see [18])

$$
\begin{gathered}
{[\mathbf{L} \mathbf{U}]_{i}=\sum_{j, k} \frac{\partial}{\partial y_{j}}\left(g^{j k} \frac{\partial U_{i}}{\partial y_{k}}\right)+2 \sum_{j, k, l} g^{k l} \Gamma_{j k}^{i} \frac{\partial U_{j}}{\partial y_{l}}} \\
+\sum_{j, k, l}\left\{\frac{\partial}{\partial y_{k}}\left(g^{k l} \Gamma_{j l}^{i}\right)+\sum_{m} g^{k l} \Gamma_{j l}^{m} \Gamma_{k m}^{i}\right\} U_{j}, \\
{[\mathbf{G} P]_{i}=\sum_{j=1}^{2} g^{i j} \frac{\partial P}{\partial y_{j}},}
\end{gathered}
$$

where we denote (see, for instance, [7])

$$
\begin{gathered}
g^{i j}=\sum_{k} \frac{\partial Y_{i}}{\partial x_{k}} \frac{\partial Y_{j}}{\partial x_{k}} \quad \text { (metric contravariant tensor) } \\
g_{i j}=\sum_{k} \frac{\partial X_{i}}{\partial y_{k}} \frac{\partial X_{j}}{\partial y_{k}} \quad \text { (metric covariant tensor) }
\end{gathered}
$$

and

$$
\Gamma_{i j}^{k}=\frac{1}{2} \sum_{l} g^{k l}\left\{\frac{\partial g_{i l}}{\partial y_{j}}+\frac{\partial g_{j l}}{\partial y_{i}}+\frac{\partial g_{i j}}{\partial y_{l}}\right\} \quad \text { (Christoffel symbol). }
$$

We are now in position to write down the expressions of $\Delta \mathbf{u}$ and of $\nabla p$ after change of variables (see again [18] for details). 
Proposition 5.4. Suppose that

$$
(\mathbf{u}, p) \in \mathcal{H}^{2}\left(\mathcal{O} \backslash B\left(\boldsymbol{\zeta}_{1}\right)\right) \times H^{1}\left(\mathcal{O} \backslash B\left(\boldsymbol{\zeta}_{1}\right)\right) .
$$

Then, we have that

$$
(\mathbf{U}, P) \in \mathcal{H}^{2}\left(\mathcal{O} \backslash B\left(\boldsymbol{\zeta}_{2}\right)\right) \times H^{1}\left(\mathcal{O} \backslash B\left(\boldsymbol{\zeta}_{2}\right)\right) .
$$

Moreover, for all $\mathbf{y} \in \mathcal{O} \backslash B\left(\boldsymbol{\zeta}_{2}\right)$, we have that

$$
[\mathbf{L U}](\mathbf{y})=\mathbf{J}_{\mathbf{Y}}(\mathbf{X}(\mathbf{y}))[(\Delta \mathbf{u}) \circ \mathbf{X}](\mathbf{y}), \quad[\mathbf{G} P](\mathbf{y})=\mathbf{J}_{\mathbf{Y}}(\mathbf{X}(\mathbf{y}))[(\nabla p) \circ \mathbf{X}](\mathbf{y})
$$

In the remaining part of this section, we denote by $C$ a positive constant which may depend only on $\xi$ and $\mathcal{O}$. We give below (without proofs) several estimates on the dependence of the change of variables defined in (5.4) on the points $\boldsymbol{\zeta}_{1}$ and $\boldsymbol{\zeta}_{2}$. For the proofs of these estimates, we refer to [27] and to [28].

Lemma 5.5. Let $\boldsymbol{\Lambda}$ be the function defined by (5.2). Then, for all $\boldsymbol{\zeta}_{1}, \boldsymbol{\zeta}_{2} \in \mathcal{O}$ satisfying (5.1) we have:

$$
\begin{gathered}
\|\boldsymbol{\Lambda}\|_{\mathcal{L}^{\infty}(\mathcal{O})} \leqslant C\left|\boldsymbol{\zeta}_{1}-\boldsymbol{\zeta}_{2}\right|, \quad\|\nabla \boldsymbol{\Lambda}\|_{\left[L^{\infty}(\mathcal{O})\right]^{4}} \leqslant C\left|\boldsymbol{\zeta}_{1}-\boldsymbol{\zeta}_{2}\right| \\
\left\|\frac{\partial^{2} \boldsymbol{\Lambda}}{\partial x_{i} \partial x_{j}}\right\|_{\mathcal{L}^{\infty}(\mathcal{O})} \leqslant C\left|\boldsymbol{\zeta}_{1}-\boldsymbol{\zeta}_{2}\right|,\left\|\frac{\partial^{3} \boldsymbol{\Lambda}}{\partial x_{i} \partial x_{j} \partial x_{k}}\right\|_{\mathcal{L}^{\infty}(\mathcal{O})} \leqslant C\left|\boldsymbol{\zeta}_{1}-\boldsymbol{\zeta}_{2}\right| .
\end{gathered}
$$

Lemma 5.6. Let $\boldsymbol{\Lambda}, \boldsymbol{\zeta}_{1}, \boldsymbol{\zeta}_{2}$ be as in Lemma 5.5. Then the functions $\mathbf{X}$ and $\mathbf{Y}$ defined by (5.4) and (5.6) satisfy the following inequalities:

$$
\begin{gathered}
\|\mathbf{X}\|_{\mathcal{L}^{\infty}(\mathcal{O})} \leqslant C, \quad\|\mathbf{Y}\|_{\mathcal{L}^{\infty}(\mathcal{O})} \leqslant C, \\
\left\|\mathbf{J}_{\mathbf{X}}-\mathbf{I d}\right\|_{\left[L^{\infty}(\mathcal{O})\right]^{4}} \leqslant C\left|\boldsymbol{\zeta}_{1}-\boldsymbol{\zeta}_{2}\right|, \quad\left\|\mathbf{J}_{\mathbf{Y}}-\mathbf{I d}\right\|_{\left[L^{\infty}(\mathcal{O})\right]^{4}} \leqslant C\left|\boldsymbol{\zeta}_{1}-\boldsymbol{\zeta}_{2}\right|, \\
\left\|\frac{\partial^{2} Y_{i}}{\partial x_{j} \partial x_{k}}\right\|_{L^{\infty}(\mathcal{O})} \leqslant C\left|\boldsymbol{\zeta}_{1}-\boldsymbol{\zeta}_{2}\right|, \quad\left\|\frac{\partial^{2} X_{i}}{\partial y_{j} \partial y_{k}}\right\|_{L^{\infty}(\mathcal{O})} \leqslant C\left|\boldsymbol{\zeta}_{1}-\boldsymbol{\zeta}_{2}\right|, \\
\left\|\frac{\partial^{3} Y_{i}}{\partial x_{j} \partial x_{l} \partial x_{k}}\right\|_{L^{\infty}(\mathcal{O})} \leqslant C\left|\boldsymbol{\zeta}_{1}-\boldsymbol{\zeta}_{2}\right|, \quad\left\|\frac{\partial^{3} X_{i}}{\partial y_{j} \partial y_{l} \partial y_{k}}\right\|_{L^{\infty}(\mathcal{O})} \leqslant C\left|\boldsymbol{\zeta}_{1}-\boldsymbol{\zeta}_{2}\right| .
\end{gathered}
$$

Lemma 5.7. Let $\boldsymbol{\Lambda}, \boldsymbol{\zeta}_{1}, \boldsymbol{\zeta}_{2}$ be as in Lemma 5.5. Moreover, suppose that

$$
(\mathbf{U}, P) \in \mathcal{H}^{2}\left(\mathcal{O} \backslash B\left(\boldsymbol{\zeta}_{2}\right)\right) \times H^{1}\left(\mathcal{O} \backslash B\left(\boldsymbol{\zeta}_{2}\right)\right)
$$

and that $\mathbf{L}$ and $\mathbf{G}$ are given by (5.11) and (5.12). Then we have

(i) $\|\nu[(\mathbf{L}-\Delta) \mathbf{U}]\|_{\mathcal{L}^{2}\left(\mathcal{O} \backslash B\left(\boldsymbol{\zeta}_{2}\right)\right)} \leqslant C\left|\boldsymbol{\zeta}_{1}-\boldsymbol{\zeta}_{2}\right|\|\mathbf{U}\|_{\mathcal{H}^{2}\left(\mathcal{O} \backslash B\left(\boldsymbol{\zeta}_{2}\right)\right)}$

(ii) $\quad\|[(\nabla-\mathbf{G}) P]\|_{\mathcal{L}^{2}\left(\mathcal{O} \backslash B\left(\boldsymbol{\zeta}_{2}\right)\right)} \leqslant C\left|\boldsymbol{\zeta}_{1}-\boldsymbol{\zeta}_{2}\right|\|P\|_{H^{1}\left(\mathcal{O} \backslash B\left(\boldsymbol{\zeta}_{2}\right)\right)}$ 


\section{Consistency of the fully discretized scheme.}

This section is devoted to the consistency of our fully discretized scheme. The main result in this section asserts that the solution $(\mathbf{u}, p, \boldsymbol{\zeta}, \omega)$ of (1.1)-(1.8) satisfies the scheme (3.2)-(3.6) with consistency errors that will be estimated. Since $\left(\mathbf{u}\left(t_{k}\right), p\left(t_{k}\right)\right)$ belongs to $\mathcal{K}\left(\boldsymbol{\zeta}\left(t_{k}\right)\right) \times M\left(\boldsymbol{\zeta}\left(t_{k}\right)\right)$ and not to $\mathcal{K}\left(\boldsymbol{\zeta}_{h}^{k}\right) \times M\left(\boldsymbol{\zeta}_{h}^{k}\right)$, we need the change of variables introduced in the previous section.

\subsection{Consistency in time.}

In this subsection we show that the exact values at instants $t=t_{k}$ of a strong solution of (1.1)-(1.8) satisfy a perturbed version of the semi-discretized problem introduced in Subsection 2.2 and we estimate these perturbations with respect to the time step. The precise statement is given in Lemma 6.1 below.

Consider the solution $(\mathbf{u}, p, \boldsymbol{\zeta}, \omega)$ of (1.1)-(1.8) and assume (3.8) and (3.10) hold. In the sequel, we will use the notation:

$$
\widetilde{\boldsymbol{X}}(\mathbf{x})=\widetilde{\boldsymbol{\psi}}\left(t_{k} ; t_{k+1}, \mathbf{x}\right) \quad \forall \mathbf{x} \in \mathcal{O}
$$

where $\widetilde{\boldsymbol{\psi}}$ is defined by the relation $(2.6)$. Note that $\widetilde{\boldsymbol{X}}(\mathcal{O})=\mathcal{O}$.

Let $\boldsymbol{\varepsilon}_{k}, \boldsymbol{\delta}_{k}, \boldsymbol{\alpha}_{k}, \boldsymbol{\beta}_{k}, \gamma_{k}$ be quantities defined by:

$$
\begin{aligned}
\boldsymbol{\varepsilon}_{k} & =\boldsymbol{\zeta}\left(t_{k+1}\right)-\boldsymbol{\zeta}\left(t_{k}\right)-\boldsymbol{\zeta}^{\prime}\left(t_{k}\right) \Delta t \\
\boldsymbol{\delta}_{k}(t, \mathbf{x}) & =\mathbf{u}\left(\widetilde{\boldsymbol{\psi}}\left(t ; t_{k+1}, \mathbf{x}\right), t\right)-\mathbf{u}\left(\widetilde{\boldsymbol{\psi}}\left(t ; t_{k+1}, \mathbf{x}\right), t_{k}\right), \\
\boldsymbol{\alpha}_{k} & =\frac{\mathbf{u}\left(t_{k+1}\right)-\mathbf{u}\left(t_{k}\right) \circ \widetilde{\boldsymbol{X}}}{\Delta t}-\frac{d}{d t}[\mathbf{u} \circ \widetilde{\boldsymbol{\psi}}]\left(t_{k+1}\right), \\
\boldsymbol{\beta}_{k} & =\frac{\boldsymbol{\zeta}^{\prime}\left(t_{k+1}\right)-\boldsymbol{\zeta}^{\prime}\left(t_{k}\right)}{\Delta t}-\boldsymbol{\zeta}^{\prime \prime}\left(t_{k+1}\right) \\
\gamma_{k} & =\frac{\omega^{\prime}\left(t_{k+1}\right)-\omega^{\prime}\left(t_{k}\right)}{\Delta t}-\omega^{\prime \prime}\left(t_{k+1}\right) .
\end{aligned}
$$

By using the fact that $\mathbf{u}\left(\boldsymbol{\zeta}\left(t_{k}\right), t_{k}\right)=\boldsymbol{\zeta}^{\prime}\left(t_{k}\right)$ and relations (2.6), (1.1), (1.5) and (1.6) together with the above definitions, we infer that the exact solution $(\mathbf{u}, p, \boldsymbol{\zeta}, \omega)$ satisfies

$$
\begin{gathered}
\boldsymbol{\zeta}\left(t_{k+1}\right)=\boldsymbol{\zeta}\left(t_{k}\right)+\mathbf{u}\left(\boldsymbol{\zeta}\left(t_{k}\right), t_{k}\right) \Delta t+\boldsymbol{\varepsilon}_{k} \\
\left\{\begin{array}{l}
\frac{d}{d t} \widetilde{\boldsymbol{\psi}}\left(t ; t_{k+1}, \mathbf{x}\right)=\mathbf{u}\left(\widetilde{\boldsymbol{\psi}}\left(t ; t_{k+1}, \mathbf{x}\right), t_{k}\right)+\boldsymbol{\delta}_{k}(t, \mathbf{x}), \\
\widetilde{\boldsymbol{\psi}}\left(t_{k+1} ; t_{k+1}, \mathbf{x}\right)=\mathbf{x}
\end{array}\right.
\end{gathered}
$$

for all $\mathbf{x} \in \mathcal{O}$ and for all $t \in\left[t_{k}, t_{k+1}\right]$, together with

$$
\frac{\mathbf{u}\left(t_{k+1}\right)-\mathbf{u}\left(t_{k}\right) \circ \widetilde{\boldsymbol{X}}}{\Delta t}-\nu \Delta \mathbf{u}\left(t_{k+1}\right)+\nabla p\left(t_{k+1}\right)=\mathbf{f}^{k+1}+\boldsymbol{\alpha}_{k}, \quad \text { in } \mathcal{O} \backslash B\left(\boldsymbol{\zeta}\left(t_{k+1}\right)\right),
$$




$$
\begin{aligned}
& M \frac{\boldsymbol{\zeta}^{\prime}\left(t_{k+1}\right)-\boldsymbol{\zeta}^{\prime}\left(t_{k}\right)}{\Delta t}=-\int_{\partial B\left(\boldsymbol{\zeta}\left(t_{k+1}\right)\right)} \boldsymbol{\sigma}\left(t_{k+1}\right) \mathbf{n} \mathrm{d} \Gamma+\int_{B\left(\boldsymbol{\zeta}\left(t_{k+1}\right)\right)} \mathbf{f}^{k+1} \mathrm{~d} \mathbf{x}+\boldsymbol{\beta}_{k}, \\
& J \frac{\omega\left(t_{k+1}\right)-\omega\left(t_{k}\right)}{\Delta t}=-\int_{\partial B\left(\boldsymbol{\zeta}\left(t_{k+1}\right)\right)}\left(\mathbf{y}-\boldsymbol{\zeta}\left(t_{k+1}\right)\right)^{\perp} \cdot \boldsymbol{\sigma}\left(t_{k+1}\right) \mathbf{n} \mathrm{d} \Gamma \\
& +\int_{B\left(\boldsymbol{\zeta}\left(t_{k+1}\right)\right)}\left(\mathbf{y}-\boldsymbol{\zeta}\left(t_{k+1}\right)\right)^{\perp} \cdot \mathbf{f}^{k+1} \mathrm{~d} \mathbf{x}+\gamma_{k} .
\end{aligned}
$$

Moreover, if we denote

$$
\theta(t)=\int_{0}^{t} \omega(s) \mathrm{d} s
$$

and by $\mathbf{R}_{\theta}$ the rotation matrix of angle $\theta$, then we also define the matrix $\mathbf{E}_{k}$ by

$$
\mathbf{R}_{\theta\left(t_{k+1}\right)-\theta\left(t_{k}\right)}=\mathbf{I d}-\Delta t \omega\left(t_{k+1}\right) \mathbf{R}_{-\pi / 2}+\mathbf{E}_{k}
$$

By using the Taylor-Lagrange inequality, we easily obtain the following consistency error estimates.

Lemma 6.1. The elements $\boldsymbol{\alpha}_{k}, \boldsymbol{\beta}_{k}, \gamma_{k}, \boldsymbol{\delta}_{k}, \boldsymbol{\varepsilon}_{k}$ and $\mathbf{E}_{k}$ defined by (6.2)-(6.6) satisfy the following inequalities:

$$
\begin{gathered}
\left|\varepsilon_{k}\right| \leqslant C(\Delta t)^{2}, \quad\left\|\boldsymbol{\delta}_{k}\right\|_{\mathcal{L}^{2}\left(\mathcal{O} \times\left(t_{k}, t_{k+1}\right)\right)} \leqslant C \Delta t\left\|\frac{\partial \mathbf{u}}{\partial t}\right\|_{\mathcal{L}^{2}\left(\mathcal{O} \times\left(t_{k}, t_{k+1}\right)\right)} \\
\left\|\boldsymbol{\alpha}_{k}\right\|_{\mathcal{L}^{2}(\mathcal{O})} \leqslant C \sqrt{\Delta t}\left\|\frac{d^{2}}{d t^{2}}[\mathbf{u} \circ \widetilde{\boldsymbol{\psi}}]\right\|_{\mathcal{L}^{2}\left(\mathcal{O} \times\left(t_{k}, t_{k+1}\right)\right)}, \\
\left|\boldsymbol{\beta}_{k}\right| \leqslant C \Delta t, \quad\left|\gamma_{k}\right| \leqslant C \Delta t, \quad\left|\mathbf{E}_{k}\right| \leqslant C(\Delta t)^{2} .
\end{gathered}
$$

\subsection{Transformed system.}

We need to compare $\mathbf{u}\left(t_{k}\right) \in \mathcal{K}\left(\boldsymbol{\zeta}\left(t_{k}\right)\right)$, which is a rigid velocity field in $B\left(\boldsymbol{\zeta}\left(t_{k}\right)\right)$ with $\mathbf{u}_{h}^{k} \in \mathcal{K}\left(\boldsymbol{\zeta}_{h}^{k}\right)$ which is a rigid velocity field in $B\left(\boldsymbol{\zeta}_{h}^{k}\right)$. This will be done by using the change of variables introduced in Section 5.1. To this end, we suppose that $\left|\boldsymbol{\zeta}_{h}^{k}-\boldsymbol{\zeta}\left(t_{k}\right)\right|<\eta$. This hypothesis and (3.10) imply that

$$
\operatorname{dist}\left(B\left(\boldsymbol{\zeta}\left(t_{k}\right)\right), \partial \mathcal{O}\right)>2 \eta \text {. }
$$

With this assumption, we can transform $\mathbf{u}\left(t_{k}\right)$ by using the change of variables introduced in Section 5.1: we denote (see (5.4), (5.6))

$$
\mathbf{X}^{k}=\mathbf{X}_{\boldsymbol{\zeta}_{h}^{k}, \boldsymbol{\zeta}\left(t_{k}\right)}, \quad \mathbf{Y}^{k}=\mathbf{Y}_{\boldsymbol{\zeta}_{h}^{k}, \boldsymbol{\zeta}\left(t_{k}\right)} .
$$

We also define (see (5.7) and (5.8))

$$
\begin{gathered}
\mathbf{U}^{k}(\mathbf{y})=\mathbf{J}_{\mathbf{Y}^{k}}\left(\mathbf{X}^{k}(\mathbf{y})\right) \mathbf{u}\left(\mathbf{X}^{k}(\mathbf{y}), t_{k}\right), \quad P^{k}(\mathbf{y})=p^{k}\left(\mathbf{X}^{k}(\mathbf{y})\right), \\
\boldsymbol{S}^{k}=-P^{k} \mathbf{I} \mathbf{d}+2 \nu \mathbf{D}\left(\mathbf{U}^{k}\right), \quad \mathbf{F}^{k}(\mathbf{y})=\mathbf{J}_{\mathbf{Y}^{k}}\left(\mathbf{X}^{k}(\mathbf{y})\right) \mathbf{f}\left(\mathbf{X}^{k}(\mathbf{y}), t_{k}\right) .
\end{gathered}
$$


We recall that, according to Lemma 5.3, $\mathbf{U}^{k} \in \widehat{\mathcal{K}}\left(\boldsymbol{\zeta}_{h}^{k}\right)$ and that $P^{k} \in M\left(\boldsymbol{\zeta}_{h}^{k}\right)$. We introduce the following notations that will be useful in the sequel:

$$
\widehat{\boldsymbol{X}}=\mathbf{Y}^{k} \circ \widetilde{\boldsymbol{X}} \circ \mathbf{X}^{k+1}
$$

and

$$
\widehat{\mathbf{J}}=\left(\mathbf{J}_{\mathbf{Y}^{k+1}} \circ \mathbf{X}^{k+1}\right)\left(\mathbf{J}_{\mathbf{X}^{k}} \circ \widehat{\boldsymbol{X}}\right)
$$

Before stating the main result of this section, let us give some properties on the characteristics. First note that, according to Lemma 5.2, we have

$$
\begin{gathered}
\mathbf{X}^{k+1}\left(B\left(\boldsymbol{\zeta}_{h}^{k+1}\right)\right)=B\left(\boldsymbol{\zeta}\left(t_{k+1}\right)\right) \\
\mathbf{Y}^{k}\left(B\left(\boldsymbol{\zeta}\left(t_{k}\right)\right)\right)=B\left(\boldsymbol{\zeta}_{h}^{k}\right),
\end{gathered}
$$

Moreover, we can easily check that the function $\widetilde{\boldsymbol{X}}$ defined by (6.1) satisfies

$$
\widetilde{\boldsymbol{X}}(\mathbf{x})=\boldsymbol{\zeta}\left(t_{k}\right)+\mathbf{R}_{\theta\left(t_{k+1}\right)-\theta\left(t_{k}\right)}\left(\mathbf{x}-\boldsymbol{\zeta}\left(t_{k+1}\right)\right) \quad \forall \mathbf{x} \in B\left(\boldsymbol{\zeta}\left(t_{k+1}\right)\right) .
$$

Consequently, we have

$$
\widetilde{\boldsymbol{X}}\left(B\left(\boldsymbol{\zeta}\left(t_{k+1}\right)\right)\right)=B\left(\boldsymbol{\zeta}\left(t_{k}\right)\right)
$$

and therefore, we obtain

$$
\widehat{\boldsymbol{X}}\left(B\left(\boldsymbol{\zeta}_{h}^{k+1}\right)\right)=B\left(\boldsymbol{\zeta}_{h}^{k}\right)
$$

We summarize some of the above properties in the following diagram:

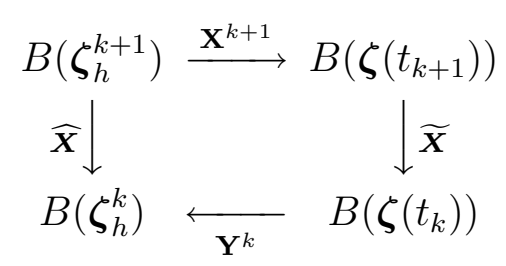

Next, we turn to the main result of this subsection: we show that $\mathbf{U}^{k+1}$ and $P^{k+1}$ satisfy a mixed weak formulation with test functions in $\mathcal{K}\left(\boldsymbol{\zeta}_{h}^{k+1}\right)$ and $M\left(\boldsymbol{\zeta}_{h}^{k+1}\right)$.

Proposition 6.2. The functions $\left(\mathbf{U}^{k+1}, P^{k+1}\right)$ defined by (6.16) satisfy

$$
\begin{gathered}
\left(\frac{1}{\Delta t}\left[\mathbf{U}^{k+1}-\widehat{\mathbf{J}}\left(\mathbf{U}^{k} \circ \widehat{\boldsymbol{X}}\right)\right], \boldsymbol{\varphi}\right)+a\left(\mathbf{U}^{k+1}, \boldsymbol{\varphi}\right)+b\left(\boldsymbol{\varphi}, P^{k+1}\right) \\
=\left(\mathbf{f}_{h}^{k+1}, \boldsymbol{\varphi}\right)+\left(\mathbf{A}_{k}, \boldsymbol{\varphi}\right) \quad \forall \boldsymbol{\varphi} \in \mathcal{K}\left(\boldsymbol{\zeta}_{h}^{k+1}\right), \\
b\left(\mathbf{U}^{k+1}, q\right)=0 \quad \forall q \in M\left(\boldsymbol{\zeta}_{h}^{k+1}\right),
\end{gathered}
$$

with

$$
\left\|\mathbf{A}_{k}\right\|_{\mathcal{L}^{2}(\mathcal{O})} \leqslant C\left(\left|\boldsymbol{\zeta}\left(t_{k+1}\right)-\boldsymbol{\zeta}_{h}^{k+1}\right|+h+\Delta t+C \sqrt{\Delta t}\left\|\frac{d^{2}}{d t^{2}}[\mathbf{u} \circ \widetilde{\boldsymbol{\psi}}]\right\|_{\mathcal{L}^{2}\left(\mathcal{O} \times\left(t_{k}, t_{k+1}\right)\right)}\right) .
$$


Proof.

First Step: Transformation of the equation (6.9).

By using Proposition 5.4, we have that $\mathbf{U}^{k+1}$ and $P^{k+1}$ satisfy

$$
\begin{gathered}
\left(\mathbf{J}_{\mathbf{Y}^{k+1}} \circ \mathbf{X}^{k+1}\right) \frac{\mathbf{u}\left(t_{k+1}\right)-\mathbf{u}\left(t_{k}\right) \circ \widetilde{\boldsymbol{X}}}{\Delta t} \circ \mathbf{X}^{k+1}-\nu\left[\mathbf{L}^{k+1} \mathbf{U}^{k+1}\right]+\left[\mathbf{G}^{k+1} P^{k+1}\right] \\
=\left(\mathbf{J}_{\mathbf{Y}^{k+1}} \circ \mathbf{X}^{k+1}\right)\left(\mathbf{f}\left(\mathbf{X}^{k+1}, t_{k+1}\right)\right)+\left(\mathbf{J}_{\mathbf{Y}^{k+1}} \circ \mathbf{X}^{k+1}\right)\left(\boldsymbol{\alpha}_{k+1} \circ \mathbf{X}^{k+1}\right), \\
\quad \text { in } \mathcal{O} \backslash B\left(\boldsymbol{\zeta}_{h}^{k+1}\right) .
\end{gathered}
$$

The above relation and (6.16) imply

$$
\begin{aligned}
& \frac{1}{\Delta t}\left[\mathbf{U}^{k+1}-\left(\mathbf{J}_{\mathbf{Y}^{k+1}} \circ \mathbf{X}^{k+1}\right)\left(\mathbf{J}_{\mathbf{X}^{k}} \circ \widehat{\boldsymbol{X}}\right)\left(\mathbf{U}^{k} \circ \widehat{\boldsymbol{X}}\right)\right]-\nu \Delta \mathbf{U}^{k+1}+\nabla P^{k+1} \\
& =\nu\left[\left(\mathbf{L}^{k+1}-\Delta\right) \mathbf{U}^{k+1}\right]+\left[\left(\nabla-\mathbf{G}^{k+1}\right) P^{k+1}\right]+\mathbf{F}^{k+1}+\left(\mathbf{J}_{\mathbf{Y}^{k+1}} \circ \mathbf{X}^{k+1}\right)\left(\boldsymbol{\alpha}_{k+1} \circ \mathbf{X}^{k+1}\right), \\
& \text { in } \mathcal{O} \backslash B\left(\boldsymbol{\zeta}_{h}^{k+1}\right),
\end{aligned}
$$

where $\widehat{\boldsymbol{X}}$ is defined by $(6.17)$

By taking the inner product of the previous equation with $\varphi \in \mathcal{K}\left(\boldsymbol{\zeta}_{h}^{k+1}\right)$ and by using (6.18), we obtain

$$
\begin{aligned}
\int_{\mathcal{O} \backslash B\left(\boldsymbol{\zeta}_{h}^{k+1}\right)}\left(\frac{1}{\Delta t}\left[\mathbf{U}^{k+1}-\widehat{\mathbf{J}}\left(\mathbf{U}^{k} \circ \widehat{\boldsymbol{X}}\right)\right] \cdot \boldsymbol{\varphi}\right) \mathrm{d} \mathbf{y} & \\
& \quad-\int_{\mathcal{O} \backslash B\left(\boldsymbol{\zeta}_{h}^{k+1}\right)}\left(\operatorname{div} \boldsymbol{S}^{k+1} \cdot \boldsymbol{\varphi}\right) \mathrm{d} \mathbf{y}=\int_{\mathcal{O} \backslash B\left(\boldsymbol{\zeta}_{h}^{k+1}\right)} \mathbf{F}^{k+1} \cdot \boldsymbol{\varphi} \mathrm{d} \mathbf{y}+A_{1}
\end{aligned}
$$

with

$$
\begin{aligned}
& A_{1}=\int_{\mathcal{O} \backslash B\left(\boldsymbol{\zeta}_{h}^{k+1}\right)}\left(\nu\left[\left(\mathbf{L}^{k+1}-\Delta\right) \mathbf{U}^{k+1}\right]+\left[\left(\nabla-\mathbf{G}^{k+1}\right) P^{k+1}\right]\right) \cdot \boldsymbol{\varphi} \mathrm{d} \mathbf{y} \\
&\left.+\int_{\mathcal{O} \backslash B\left(\boldsymbol{\zeta}_{h}^{k+1}\right)}\left(\mathbf{J}_{\mathbf{Y}^{k+1}} \circ \mathbf{X}^{k+1}\right)\left(\boldsymbol{\alpha}_{k+1} \circ \mathbf{X}^{k+1}\right)\right) \cdot \boldsymbol{\varphi} \mathrm{d} \mathbf{y} .
\end{aligned}
$$

Second Step: We transform the integral

$$
\int_{B\left(\boldsymbol{\zeta}_{h}^{k+1}\right)} \frac{\mathbf{U}^{k+1}-\widehat{\mathbf{J}}\left(\mathbf{U}^{k} \circ \widehat{\boldsymbol{X}}\right)}{\Delta t} \cdot \boldsymbol{\varphi} \mathrm{d} \mathbf{y} .
$$

by using equations (6.10)-(6.11). From (5.3) (with $Y$ as in (6.15)), combined to (5.9) and to (5.10) we obtain that:

$$
\mathbf{J}_{\mathbf{Y}^{k+1}}(\mathbf{x})=\mathbf{I d} \quad \forall \mathbf{x} \in B\left(\boldsymbol{\zeta}\left(t_{k+1}\right)\right) .
$$

The above relation, (6.16) and (5.9) imply that for all $\mathbf{y} \in B\left(\boldsymbol{\zeta}_{h}^{k+1}\right)$,

$$
\mathbf{U}^{k+1}(\mathbf{y})=\mathbf{u}\left(\mathbf{y}+\boldsymbol{\zeta}\left(t_{k+1}\right)-\boldsymbol{\zeta}_{h}^{k+1}, t_{k+1}\right) .
$$


In particular, we have that

$$
\mathbf{U}^{k+1}(\mathbf{y})=\boldsymbol{\zeta}^{\prime}\left(t_{k+1}\right)+\omega\left(t_{k+1}\right)\left(\mathbf{y}-\boldsymbol{\zeta}_{h}^{k+1}\right)^{\perp} \quad \forall \mathbf{y} \in B\left(\boldsymbol{\zeta}_{h}^{k+1}\right) .
$$

Similarly, we have

$$
\mathbf{U}^{k}(\mathbf{y})=\boldsymbol{\zeta}^{\prime}\left(t_{k}\right)+\omega\left(t_{k}\right)\left(\mathbf{y}-\boldsymbol{\zeta}_{h}^{k}\right)^{\perp} \quad \forall \mathbf{y} \in B\left(\boldsymbol{\zeta}_{h}^{k}\right)
$$

Relations (6.19) and (6.21) yield

$$
\left(\mathbf{J}_{\mathbf{Y}^{k+1}} \circ \mathbf{X}^{k+1}\right)\left(\mathbf{J}_{\mathbf{X}^{k}} \circ \widehat{\boldsymbol{X}}\right)=\mathbf{I d}, \quad \text { in } \quad B\left(\boldsymbol{\zeta}_{h}^{k+1}\right) .
$$

Simple calculations combined with relations (5.9), (6.20) yield

$$
\widehat{\boldsymbol{X}}(\mathbf{y})=\mathbf{R}_{\theta\left(t_{k+1}\right)-\theta\left(t_{k}\right)}\left(\mathbf{y}-\boldsymbol{\zeta}_{h}^{k+1}\right)+\boldsymbol{\zeta}_{h}^{k} \quad \forall \mathbf{y} \in B\left(\boldsymbol{\zeta}_{h}^{k+1}\right) .
$$

The above relation, (6.32) and (6.31) imply that for all $\mathbf{y} \in B\left(\boldsymbol{\zeta}_{h}^{k+1}\right)$, we have that

$$
\widehat{\mathbf{J}}\left(\mathbf{U}^{k} \circ \widehat{\boldsymbol{X}}\right)(\mathbf{y})=\boldsymbol{\zeta}^{\prime}\left(t_{k}\right)+\omega\left(t_{k}\right) \mathbf{R}_{\theta\left(t_{k+1}\right)-\theta\left(t_{k}\right)}\left(\mathbf{y}-\boldsymbol{\zeta}_{h}^{k+1}\right)^{\perp} .
$$

By using (6.12), the previous equality can be written as

$$
\begin{aligned}
\widehat{\mathbf{J}}\left(\mathbf{U}^{k} \circ \widehat{\boldsymbol{X}}\right)(\mathbf{y})= & \boldsymbol{\zeta}^{\prime}\left(t_{k}\right)+\omega\left(t_{k}\right)\left(\mathbf{y}-\boldsymbol{\zeta}_{h}^{k+1}\right)^{\perp} \\
& +\Delta t \omega\left(t_{k}\right) \omega\left(t_{k+1}\right)\left(\mathbf{y}-\boldsymbol{\zeta}_{h}^{k+1}\right)+\omega\left(t_{k}\right) \mathbf{E}_{k}\left(\mathbf{y}-\boldsymbol{\zeta}_{h}^{k+1}\right)^{\perp} \quad \forall \mathbf{y} \in B\left(\boldsymbol{\zeta}_{h}^{k+1}\right) .
\end{aligned}
$$

By taking the inner product of the above relation with $\boldsymbol{\varphi} \in \mathcal{K}\left(\boldsymbol{\zeta}_{h}^{k+1}\right)$ and by integrating on $B\left(\boldsymbol{\zeta}_{h}^{k+1}\right)$, we obtain that

$$
\begin{aligned}
\int_{B\left(\boldsymbol{\zeta}_{h}^{k+1}\right)} \widehat{\mathbf{J}}\left(\mathbf{U}^{k} \circ \widehat{\boldsymbol{X}}\right)(\mathbf{y}) \cdot \boldsymbol{\varphi} \mathrm{d} \mathbf{y}=M \mathbf{l}_{\boldsymbol{\varphi}} \cdot \boldsymbol{\zeta}^{\prime}\left(t_{k}\right)+J \omega\left(t_{k}\right) \omega_{\boldsymbol{\varphi}} & \\
& +\omega\left(t_{k}\right) \int_{B\left(\boldsymbol{\zeta}_{h}^{k+1}\right)} \mathbf{E}_{k}\left(\mathbf{y}-\boldsymbol{\zeta}_{h}^{k+1}\right)^{\perp} \cdot \boldsymbol{\varphi} \mathrm{d} \mathbf{y}
\end{aligned}
$$

Relation (6.30) implies that, for all $\boldsymbol{\varphi} \in \mathcal{K}\left(\boldsymbol{\zeta}_{h}^{k+1}\right)$, we have

$$
\int_{B\left(\boldsymbol{\zeta}_{h}^{k+1}\right)} \mathbf{U}^{k+1} \cdot \boldsymbol{\varphi} \mathrm{d} \mathbf{y}=M \mathbf{l}_{\boldsymbol{\varphi}} \cdot \boldsymbol{\zeta}^{\prime}\left(t_{k+1}\right)+J \omega\left(t_{k+1}\right) \omega_{\boldsymbol{\varphi}}
$$

The above equality and (6.33) yield that for all $\boldsymbol{\varphi} \in \mathcal{K}\left(\boldsymbol{\zeta}_{h}^{k+1}\right)$, we have

$$
\begin{aligned}
\int_{B\left(\boldsymbol{\zeta}_{h}^{k+1}\right)} \frac{\mathbf{U}^{k+1}-\widehat{\mathbf{J}}\left(\mathbf{U}^{k} \circ \widehat{\boldsymbol{X}}\right)}{\Delta t} & \cdot \boldsymbol{\varphi} \mathrm{d} \mathbf{y}=M \mathbf{l}_{\boldsymbol{\varphi}} \cdot \frac{\boldsymbol{\zeta}^{\prime}\left(t_{k+1}\right)-\boldsymbol{\zeta}^{\prime}\left(t_{k}\right)}{\Delta t} \\
& +J \frac{\omega\left(t_{k+1}\right)-\omega\left(t_{k}\right)}{\Delta t} \omega_{\boldsymbol{\varphi}}-\frac{\omega\left(t_{k}\right)}{\Delta t} \int_{B\left(\boldsymbol{\zeta}_{h}^{k+1}\right)} \mathbf{E}_{k}\left(\mathbf{y}-\boldsymbol{\zeta}_{h}^{k+1}\right)^{\perp} \cdot \boldsymbol{\varphi} \mathrm{d} \mathbf{y} .
\end{aligned}
$$


The above relation and (6.10)-(6.11) imply that

$$
\begin{aligned}
& \int_{B\left(\boldsymbol{\zeta}_{h}^{k+1}\right)} \frac{\mathbf{U}^{k+1}-\widehat{\mathbf{J}}\left(\mathbf{U}^{k} \circ \widehat{\boldsymbol{X}}\right)}{\Delta t} \cdot \boldsymbol{\varphi} \mathrm{d} \mathbf{y}=-\mathbf{l}_{\boldsymbol{\varphi}} \cdot \int_{\partial B\left(\boldsymbol{\zeta}\left(t_{k+1}\right)\right)} \boldsymbol{\sigma}^{k+1} \mathbf{n} \mathrm{d} \Gamma \\
&-\omega_{\boldsymbol{\varphi}} \int_{\partial B\left(\boldsymbol{\zeta}\left(t_{k+1}\right)\right)}\left(\mathbf{y}-\boldsymbol{\zeta}\left(t_{k+1}\right)\right)^{\perp} \cdot \boldsymbol{\sigma}^{k+1} \mathbf{n} \mathrm{d} \Gamma+\mathbf{l}_{\boldsymbol{\varphi}} \cdot \int_{B\left(\boldsymbol{\zeta}\left(t_{k+1}\right)\right)} \mathbf{f}^{k+1} \mathrm{~d} \mathbf{x} \\
&+\omega_{\boldsymbol{\varphi}} \int_{B\left(\boldsymbol{\zeta}\left(t_{k+1}\right)\right)}\left(\mathbf{x}-\boldsymbol{\zeta}\left(t_{k+1}\right)\right)^{\perp} \cdot \mathbf{f}^{k+1}(x) \mathrm{d} \mathbf{x} \\
&+\mathbf{l}_{\boldsymbol{\varphi}} \cdot \boldsymbol{\beta}_{k}+\omega_{\boldsymbol{\varphi}} \gamma_{k}-\frac{\omega\left(t_{k}\right)}{\Delta t} \int_{B\left(\boldsymbol{\zeta}_{h}^{k+1}\right)} \mathbf{E}_{k}\left(\mathbf{y}-\boldsymbol{\zeta}_{h}^{k+1}\right)^{\perp} \cdot \boldsymbol{\varphi} \mathrm{d} \mathbf{y} .
\end{aligned}
$$

On the other hand, by using relations (5.9), (5.10) and (6.28), we easily obtain that

$$
\int_{\partial B\left(\boldsymbol{\zeta}_{h}^{k+1}\right)} \boldsymbol{S}^{k+1} \mathbf{n} \mathrm{d} \Gamma=\int_{\partial B\left(\boldsymbol{\zeta}\left(t_{k+1}\right)\right)} \boldsymbol{\sigma}^{k+1} \mathbf{n} \mathrm{d} \Gamma
$$

and that

$$
\int_{\partial B\left(\boldsymbol{\zeta}_{h}^{k+1}\right)}\left(\mathbf{y}-\boldsymbol{\zeta}_{h}^{k+1}\right)^{\perp} \cdot \boldsymbol{S}^{k+1} \mathbf{n} \mathrm{d} \Gamma=\int_{\partial B\left(\boldsymbol{\zeta}\left(t_{k+1}\right)\right)}\left(\mathbf{y}-\boldsymbol{\zeta}\left(t_{k+1}\right)\right)^{\perp} \cdot \boldsymbol{\sigma}^{k+1} \mathbf{n} \mathrm{d} \Gamma .
$$

The above relations and (6.34) yield that

$$
\begin{aligned}
& \int_{B\left(\boldsymbol{\zeta}_{h}^{k+1}\right)} \frac{\mathbf{U}^{k+1}-\widehat{\mathbf{J}}\left(\mathbf{U}^{k} \circ \widehat{\boldsymbol{X}}\right)}{\Delta t} \cdot \boldsymbol{\varphi} \mathrm{d} \mathbf{y}=-\int_{\partial B\left(\boldsymbol{\zeta}_{h}^{k+1}\right)}\left(\boldsymbol{S}^{k+1} \mathbf{n}\right) \cdot \boldsymbol{\varphi} \mathrm{d} \Gamma \\
& +\int_{B\left(\boldsymbol{\zeta}_{h}^{k+1}\right)} \mathbf{F}^{k+1} \cdot \boldsymbol{\varphi} \mathrm{d} \mathbf{y}+\mathbf{l}_{\boldsymbol{\varphi}} \cdot \boldsymbol{\beta}_{k}+\omega_{\boldsymbol{\varphi}} \gamma_{k}-\frac{\omega\left(t_{k}\right)}{\Delta t} \int_{B\left(\boldsymbol{\zeta}_{h}^{k+1}\right)} \mathbf{E}_{k}\left(\mathbf{y}-\boldsymbol{\zeta}_{h}^{k+1}\right)^{\perp} \cdot \boldsymbol{\varphi} \mathrm{d} \mathbf{y} .
\end{aligned}
$$

Third Step: By integrating by parts, we have that

$$
\begin{aligned}
2 \nu \int_{\mathcal{O} \backslash B\left(\boldsymbol{\zeta}_{h}^{k+1}\right)} \mathbf{D}\left(\mathbf{U}^{k+1}\right) & : \mathbf{D}(\boldsymbol{\varphi}) \mathrm{d} \mathbf{y}-\int_{\mathcal{O} \backslash B\left(\boldsymbol{\zeta}_{h}^{k+1}\right)} P^{k+1} \operatorname{div}(\boldsymbol{\varphi}) \mathrm{d} \mathbf{y} \\
= & \int_{\partial B\left(\boldsymbol{\zeta}_{h}^{k+1}\right)}\left(\boldsymbol{S}^{k+1} \mathbf{n}\right) \cdot \boldsymbol{\varphi} \mathrm{d} \Gamma-\int_{\mathcal{O} \backslash B\left(\boldsymbol{\zeta}_{h}^{k+1}\right)} \operatorname{div}\left(\boldsymbol{S}^{k+1}\right) \cdot \boldsymbol{\varphi} \mathrm{d} \mathbf{y} .
\end{aligned}
$$

Summing (6.36), (6.35) and (6.26) yields (6.22) with

$$
\begin{aligned}
\left(\mathbf{A}_{k}, \boldsymbol{\varphi}\right)=\left(\mathbf{F}^{k+1}-\mathbf{f}_{h}^{k+1}, \boldsymbol{\varphi}\right)+\mathbf{l}_{\boldsymbol{\varphi}} \cdot \boldsymbol{\beta}_{k}+\omega_{\boldsymbol{\varphi}} \gamma_{k}-\frac{\omega\left(t_{k}\right)}{\Delta t} \int_{B\left(\boldsymbol{\zeta}_{h}^{k+1}\right)} \mathbf{E}_{k}\left(\mathbf{y}-\boldsymbol{\zeta}_{h}^{k+1}\right)^{\perp} \cdot \boldsymbol{\varphi} \mathrm{d} \mathbf{y} \\
+\int_{\mathcal{O} \backslash B\left(\boldsymbol{\zeta}_{h}^{k+1}\right)}\left(\nu\left[\left(\mathbf{L}^{k+1}-\Delta\right) \mathbf{U}^{k+1}\right]+\left[\left(\nabla-\mathbf{G}^{k+1}\right) P^{k+1}\right]\right) \cdot \boldsymbol{\varphi} \mathrm{d} \mathbf{y} \\
+\int_{\mathcal{O} \backslash B\left(\boldsymbol{\zeta}_{h}^{k+1}\right)}\left(\mathbf{J}_{\mathbf{Y}^{k+1}} \circ \mathbf{X}^{k+1}\right)\left(\boldsymbol{\alpha}_{k+1} \circ \mathbf{X}^{k+1}\right) \cdot \boldsymbol{\varphi} \mathrm{d} \mathbf{y} .
\end{aligned}
$$

The above relation, combined with relation (3.7), Lemma 5.6, Lemma 5.7 and Lemma 6.1, imply the Proposition. 


\subsection{Some results on characteristics.}

In this subsection, we give some results on the functions $\mathbf{X}^{k}, \widehat{\boldsymbol{X}}$ and $\overline{\boldsymbol{X}}_{\boldsymbol{h}}^{\boldsymbol{k}}$, that will be used in the proof of the main result.

Lemma 6.3. There exists a positive constant $C$ independent of $h$ and $k$ such that

$$
\left\|\mathbf{X}^{k+1}-\mathbf{X}^{k}\right\|_{\mathcal{L}^{\infty}(\mathcal{O})} \leqslant C\left(\left\|\mathbf{u}_{h}^{k}-\mathbf{U}^{k}\right\|_{\mathcal{L}^{2}(\mathcal{O})} \Delta t+\left|\varepsilon_{k}\right|\right) .
$$

Proof. We denote by $\boldsymbol{\Lambda}^{k}$ (resp. $\boldsymbol{\Lambda}^{k+1}$ ) the mapping defined by (5.2) with $\boldsymbol{\zeta}_{1}=\boldsymbol{\zeta}\left(t_{k}\right)$ and $\boldsymbol{\zeta}_{2}=\boldsymbol{\zeta}_{h}^{k}\left(\operatorname{resp} . \boldsymbol{\zeta}_{1}=\boldsymbol{\zeta}\left(t_{k+1}\right)\right.$ and $\left.\boldsymbol{\zeta}_{2}=\boldsymbol{\zeta}_{h}^{k+1}\right)$. Let $\boldsymbol{\psi}^{k}$ and $\boldsymbol{\psi}^{k+1}$ be the solution of (5.3) corresponding to the velocity fields $\Lambda^{k}$ and $\Lambda^{k+1}$ respectively.

By using (5.3), we have that

$$
\left(\boldsymbol{\psi}^{k+1}-\boldsymbol{\psi}^{k}\right)(\lambda)=\int_{0}^{\lambda} \boldsymbol{\Lambda}^{k+1}\left(\boldsymbol{\psi}^{k+1}(\mu)\right)-\boldsymbol{\Lambda}^{k}\left(\boldsymbol{\psi}^{k}(\mu)\right) \mathrm{d} \mu
$$

Therefore, by Lemma 5.5, there exists a positive constant $C$ such that for all $\lambda \in[0,1]$, we have that

$$
\left|\left(\boldsymbol{\psi}^{k+1}-\boldsymbol{\psi}^{k}\right)(\lambda)\right| \leqslant\left\|\boldsymbol{\Lambda}^{k+1}-\boldsymbol{\Lambda}^{k}\right\|_{\mathcal{L}^{\infty}(\mathcal{O})}+C \int_{0}^{\lambda}\left|\left(\boldsymbol{\psi}^{k+1}(\mu)-\boldsymbol{\psi}^{k}(\mu)\right)\right| \mathrm{d} \mu .
$$

The above inequality and Gronwall's lemma yield

$$
\left|\left(\boldsymbol{\psi}^{k+1}-\boldsymbol{\psi}^{k}\right)(\lambda)\right| \leqslant C\left\|\boldsymbol{\Lambda}^{k+1}-\boldsymbol{\Lambda}^{k}\right\|_{\mathcal{L}^{\infty}(\mathcal{O})},
$$

for all $\lambda \in[0,1]$. In particular, for $\lambda=1$, we have that

$$
\left\|\mathbf{X}^{k+1}-\mathbf{X}^{k}\right\|_{\mathcal{L}^{\infty}(\mathcal{O})} \leqslant C\left\|\Lambda^{k+1}-\Lambda^{k}\right\|_{\mathcal{L}^{\infty}(\mathcal{O})} .
$$

By using relation (5.2), there exists a positive constant $C$ such that

$$
\left\|\Lambda^{k+1}-\boldsymbol{\Lambda}^{k}\right\|_{\mathcal{L}^{\infty}(\mathcal{O})} \leqslant C\left|\boldsymbol{\zeta}\left(t_{k+1}\right)-\boldsymbol{\zeta}_{h}^{k+1}-\boldsymbol{\zeta}\left(t_{k}\right)+\boldsymbol{\zeta}_{h}^{k}\right|
$$

The above relation, combined with (3.2) and (6.7), yields

$$
\left\|\Lambda^{k+1}-\Lambda^{k}\right\|_{\mathcal{L}^{\infty}(\mathcal{O})} \leqslant C\left|\mathbf{u}_{h}^{k}\left(\boldsymbol{\zeta}_{h}^{k}\right)-\mathbf{u}\left(\boldsymbol{\zeta}\left(t_{k}\right), t_{k}\right)\right| \Delta t+C\left|\varepsilon_{k}\right|
$$

On the other hand, by (6.29), we have $\mathbf{u}\left(\boldsymbol{\zeta}\left(t_{k}\right), t_{k}\right)=\mathbf{U}^{k}\left(\boldsymbol{\zeta}_{h}^{k}\right)$ and moreover $\mathbf{u}_{h}^{k}-\mathbf{U}^{k} \in$ $\mathcal{K}\left(\boldsymbol{\zeta}_{h}^{k}\right)$. Then, owing to $(2.5)$, we readily check that

$$
\left|\mathbf{u}_{h}^{k}\left(\boldsymbol{\zeta}_{h}^{k}\right)-\mathbf{U}^{k}\left(\boldsymbol{\zeta}_{h}^{k}\right)\right| \leqslant \frac{1}{\sqrt{M}}\left\|\mathbf{u}_{h}^{k}-\mathbf{U}^{k}\right\|_{\mathcal{L}^{2}(\mathcal{O})} .
$$

Therefore, the above relation and (6.38) imply that

$$
\left\|\boldsymbol{\Lambda}^{k+1}-\Lambda^{k}\right\|_{\mathcal{L}^{\infty}(\mathcal{O})} \leqslant C\left\|\mathbf{u}_{h}^{k}-\mathbf{U}^{k}\right\|_{\mathcal{L}^{2}(\mathcal{O})} \Delta t+C\left|\varepsilon_{k}\right| .
$$

Relations (6.37) and (6.40) yield the conclusion of the lemma. 
A similar estimate holds for the jacobian matrices $\mathbf{J}_{\mathbf{X}^{k+1}}$ and $\mathbf{J}_{\mathbf{X}^{k}}$. Since the proof of this estimate is completely similar to the proof Lemma 6.3 we give below only its statement and we skip the proof.

Lemma 6.4. There exists a positive constant $C$ independent of $k$ and $h$ such that

$$
\left\|\mathbf{J}_{\mathbf{X}^{k+1}}-\mathbf{J}_{\mathbf{X}^{k}}\right\|_{\mathcal{L}^{\infty}(\mathcal{O})} \leqslant C\left(\left\|\mathbf{u}_{h}^{k}-\mathbf{U}^{k}\right\|_{\mathcal{L}^{2}(\mathcal{O})} \Delta t+\left|\varepsilon_{k}\right|\right) .
$$

The functions $\widehat{\boldsymbol{X}}$ and $\overline{\boldsymbol{X}}_{\boldsymbol{h}}^{\boldsymbol{k}}$ are close to the identity in the sense made precise below.

Lemma 6.5. The functions $\widehat{\boldsymbol{X}}$ and $\overline{\boldsymbol{X}}_{\boldsymbol{h}}^{\boldsymbol{k}}$ defined by (6.17) and (3.4) satisfy the following estimates:

$$
\begin{aligned}
& \|\widehat{\boldsymbol{X}}-\mathbf{I d}\|_{\mathcal{L}^{2}(\mathcal{O})} \leqslant C\left(\left|\varepsilon_{k}\right|+\Delta t\left\|\mathbf{U}^{k}-\mathbf{u}_{h}^{k}\right\|_{\mathcal{L}^{2}(\mathcal{O})}+\sqrt{\Delta t}\left\|\boldsymbol{\delta}_{k}\right\|_{\mathcal{L}^{2}\left(\mathcal{O} \times\left(t_{k}, t_{k+1}\right)\right)}+\Delta t\right), \\
& \left\|\widehat{\boldsymbol{X}}-\overline{\boldsymbol{X}}_{\boldsymbol{h}}^{\boldsymbol{k}}\right\|_{\mathcal{L}^{2}(\mathcal{O})} \leqslant C\left(\left|\varepsilon_{k}\right|+\Delta t\left\|\mathbf{U}^{k}-\mathbf{u}_{h}^{k}\right\|_{\mathcal{L}^{2}(\mathcal{O})}+\sqrt{\Delta t}\left\|\boldsymbol{\delta}_{k}\right\|_{\mathcal{L}^{2}\left(\mathcal{O} \times\left(t_{k}, t_{k+1}\right)\right)}+h \Delta t\right) .
\end{aligned}
$$

Proof. Let us define

$$
\widehat{\boldsymbol{\psi}}\left(t ; t_{k+1}, \mathbf{y}\right)=\mathbf{Y}^{k}\left(\widetilde{\boldsymbol{\psi}}\left(t ; t_{k+1}, \mathbf{X}^{k+1}(\mathbf{y})\right)\right),
$$

where $\widetilde{\boldsymbol{\psi}}$ is defined by $(2.6)$. Note that $\widehat{\boldsymbol{\psi}}\left(t_{k} ; t_{k+1}, \mathbf{y}\right)=\widehat{\boldsymbol{X}}(\mathbf{y})$ for all $\mathbf{y} \in \mathcal{O}$.

We have that

$$
\frac{d}{d t} \widehat{\boldsymbol{\psi}}\left(t ; t_{k+1}, \mathbf{y}\right)=\mathbf{J}_{\mathbf{Y}^{k}}\left(\widetilde{\boldsymbol{\psi}}\left(t ; t_{k+1}, \mathbf{X}^{k+1}(\mathbf{y})\right)\right) \frac{d}{d t} \widetilde{\boldsymbol{\psi}}\left(t ; t_{k+1}, \mathbf{X}^{k+1}(\mathbf{y})\right) .
$$

By using (6.8) we obtain that

$$
\begin{aligned}
& \frac{d}{d t} \widehat{\boldsymbol{\psi}}\left(t ; t_{k+1}, \mathbf{y}\right)=\left[\mathbf{J}_{\mathbf{Y}^{k}} \circ \mathbf{X}^{k}\right]\left(\widehat{\boldsymbol{\psi}}\left(t ; t_{k+1}, \mathbf{y}\right)\right)\left[\mathbf{u}\left(\mathbf{X}^{k}\left(\widehat{\boldsymbol{\psi}}\left(t ; t_{k+1}, \mathbf{y}\right)\right), t_{k}\right)\right] \\
&+\left[\mathbf{J}_{\mathbf{Y}^{k}} \circ \mathbf{X}^{k}\right]\left(\widehat{\boldsymbol{\psi}}\left(t ; t_{k+1}, \mathbf{y}\right)\right)\left[\boldsymbol{\delta}_{k}\left(t, \mathbf{X}^{k}\left(\widehat{\boldsymbol{\psi}}\left(t ; t_{k+1}, \mathbf{y}\right)\right)\right)\right] .
\end{aligned}
$$

The above relation and (6.16) yield

$$
\begin{aligned}
\frac{d}{d t} \widehat{\boldsymbol{\psi}}\left(t ; t_{k+1}, \mathbf{y}\right)=\mathbf{U}^{k}(\widehat{\boldsymbol{\psi}}( & \left.\left.t ; t_{k+1}, \mathbf{y}\right)\right) \\
& +\left[\mathbf{J}_{\mathbf{Y}^{k}} \circ \mathbf{X}^{k}\right]\left(\widehat{\boldsymbol{\psi}}\left(t ; t_{k+1}, \mathbf{y}\right)\right)\left[\boldsymbol{\delta}_{k}\left(t, \mathbf{X}^{k}\left(\widehat{\boldsymbol{\psi}}\left(t ; t_{k+1}, \mathbf{y}\right)\right)\right)\right] .
\end{aligned}
$$

On the other hand, we have that

$$
\widehat{\boldsymbol{\psi}}\left(t_{k+1} ; t_{k+1}, \mathbf{y}\right)=\mathbf{Y}^{k} \circ \mathbf{X}^{k+1}(\mathbf{y}) .
$$

Therefore, by using (6.44) and (6.45), we get

$$
\begin{aligned}
\widehat{\boldsymbol{X}}(\mathbf{y})-\mathbf{y}=\mathbf{Y}^{k} \circ \mathbf{X}^{k+1}(\mathbf{y})-\mathbf{y}-\int_{t_{k}}^{t_{k+1}} \mathbf{U}^{k}\left(\widehat{\boldsymbol{\psi}}\left(t ; t_{k+1}, \mathbf{y}\right)\right) \mathrm{d} t \\
\quad-\int_{t_{k}}^{t_{k+1}}\left[\mathbf{J}_{\mathbf{Y}^{k}} \circ \mathbf{X}^{k}\right]\left(\widehat{\boldsymbol{\psi}}\left(t ; t_{k+1}, \mathbf{y}\right)\right)\left[\boldsymbol{\delta}_{k}\left(t, \mathbf{X}^{k}\left(\widehat{\boldsymbol{\psi}}\left(t ; t_{k+1}, \mathbf{y}\right)\right)\right)\right] \mathrm{d} t
\end{aligned}
$$


which yields

$$
\begin{aligned}
\|\widehat{\boldsymbol{X}}-\mathbf{I d}\|_{\mathcal{L}^{2}(\mathcal{O})} \leqslant \| \mathbf{Y}^{k} & \circ \mathbf{X}^{k+1}-\mathbf{I d} \|_{\mathcal{L}^{2}(\mathcal{O})} \\
& +\int_{t_{k}}^{t_{k+1}}\left\|\mathbf{U}^{k}(\widehat{\psi}(s))\right\|_{\mathcal{L}^{2}(\mathcal{O})} \mathrm{d} s+C \sqrt{\Delta t}\left\|\boldsymbol{\delta}_{k}\right\|_{\mathcal{L}^{2}\left(\mathcal{O} \times\left(t_{k}, t_{k+1}\right)\right)} .
\end{aligned}
$$

By Lemma 5.6, there exists a positive constant $C$ such that

$$
\left\|\mathbf{Y}^{k} \circ \mathbf{X}^{k+1}-\mathbf{I d}\right\|_{\mathcal{L}^{2}(\mathcal{O})} \leqslant C\left\|\mathbf{X}^{k+1}-\mathbf{X}^{k}\right\|_{\mathcal{L}^{\infty}(\mathcal{O})} .
$$

The above relation and Lemma 6.3 yield

$$
\left\|\mathbf{Y}^{k} \circ \mathbf{X}^{k+1}-\mathbf{I d}\right\|_{\mathcal{L}^{2}(\mathcal{O})} \leqslant C\left(\Delta t\left\|\mathbf{u}_{h}^{k}-\mathbf{U}^{k}\right\|_{\mathcal{L}^{2}(\mathcal{O})}+\left|\varepsilon_{k}\right|\right) .
$$

Relations (6.46) and (6.47), together with (3.8) and (6.16), imply

$$
\|\widehat{\boldsymbol{X}}-\mathbf{I d}\|_{\mathcal{L}^{2}(\mathcal{O})} \leqslant C\left(\Delta t\left\|\mathbf{u}_{h}^{k}-\mathbf{U}^{k}\right\|_{\mathcal{L}^{2}(\mathcal{O})}+\left|\varepsilon_{k}\right|\right)+C \Delta t+\sqrt{\Delta t}\left\|\boldsymbol{\delta}_{k}\right\|_{\mathcal{L}^{2}\left(\mathcal{O} \times\left(t_{k}, t_{k+1}\right)\right)} .
$$

Therefore, we deduce (6.41).

Now we turn to the proof of $(6.42)$ : by using $(3.3),(6.44)$ and $(6.45)$, we obtain that

$$
\begin{aligned}
\widehat{\boldsymbol{\psi}}\left(t ; t_{k+1}, \mathbf{y}\right)-\overline{\boldsymbol{\psi}}_{\boldsymbol{h}}^{\boldsymbol{k}}\left(t ; t_{k+1}, \mathbf{y}\right)=\mathbf{Y}^{k} \circ \mathbf{X}^{k+1}(\mathbf{y})-\mathbf{y} \\
\quad-\int_{t}^{t_{k+1}}\left(\mathbf{U}^{k}\left(\widehat{\boldsymbol{\psi}}\left(s ; t_{k+1}, \mathbf{y}\right)\right)-\mathbf{P u}_{h}^{k}\left(\overline{\boldsymbol{\psi}}_{\boldsymbol{h}}^{\boldsymbol{k}}\left(s ; t_{k+1}, \mathbf{y}\right)\right)\right) \mathrm{d} s \\
\quad-\int_{t}^{t_{k+1}}\left(\mathbf{J}_{\mathbf{Y}^{k}} \circ \mathbf{X}^{k}\right)\left(\widehat{\boldsymbol{\psi}}\left(s ; t_{k+1}, \mathbf{y}\right)\right)\left[\boldsymbol{\delta}_{k}\left(s, \mathbf{X}^{k}\left(\widehat{\boldsymbol{\psi}}\left(s ; t_{k+1}, \mathbf{y}\right)\right)\right)\right] \mathrm{d} s
\end{aligned}
$$

which yields

$$
\begin{aligned}
\| \widehat{\boldsymbol{\psi}}(t)- & \overline{\boldsymbol{\psi}}_{\boldsymbol{h}}^{\boldsymbol{k}}(t)\left\|_{\mathcal{L}^{2}(\mathcal{O})} \leqslant\right\| \mathbf{Y}^{k} \circ \mathbf{X}^{k+1}-\mathbf{I d} \|_{\mathcal{L}^{2}(\mathcal{O})} \\
& +\int_{t}^{t_{k+1}}\left\|\mathbf{U}^{k}(\widehat{\boldsymbol{\psi}}(s))-\mathbf{P u}_{h}^{k}\left(\overline{\boldsymbol{\psi}}_{\boldsymbol{h}}^{\boldsymbol{k}}(s)\right)\right\|_{\mathcal{L}^{2}(\mathcal{O})} \mathrm{d} s+C \sqrt{\Delta t}\left\|\boldsymbol{\delta}_{k}\right\|_{\mathcal{L}^{2}\left(\mathcal{O} \times\left(t_{k}, t_{k+1}\right)\right)} .
\end{aligned}
$$

Relations (6.48) and (6.47) imply

$$
\begin{aligned}
\| \widehat{\boldsymbol{\psi}}(t)-\overline{\boldsymbol{\psi}}_{\boldsymbol{h}}^{\boldsymbol{k}}(t) & \|_{\mathcal{L}^{2}(\mathcal{O})} \leqslant C\left(\Delta t\left\|\mathbf{u}_{h}^{k}-\mathbf{U}^{k}\right\|_{\mathcal{L}^{2}(\mathcal{O})}+\left|\varepsilon_{k}\right|\right) \\
& +\int_{t}^{t_{k+1}}\left\|\mathbf{U}^{k}(\widehat{\boldsymbol{\psi}}(s))-\mathbf{P} \mathbf{u}_{h}^{k}\left(\overline{\boldsymbol{\psi}}_{\boldsymbol{h}}^{\boldsymbol{k}}(s)\right)\right\|_{\mathcal{L}^{2}(\mathcal{O})} \mathrm{d} s+C \sqrt{\Delta t}\left\|\boldsymbol{\delta}_{k}\right\|_{\mathcal{L}^{2}\left(\mathcal{O} \times\left(t_{k}, t_{k+1}\right)\right)} .
\end{aligned}
$$

By using (3.8) and Remark 3.1, we have that

$$
\begin{aligned}
\left\|\widehat{\boldsymbol{\psi}}(t)-\overline{\boldsymbol{\psi}}_{\boldsymbol{h}}^{\boldsymbol{k}}(t)\right\|_{\mathcal{L}^{2}(\mathcal{O})} \leqslant & C\left(\Delta t\left\|\mathbf{u}_{h}^{k}-\mathbf{U}^{k}\right\|_{\mathcal{L}^{2}(\mathcal{O})}+\left|\varepsilon_{k}\right|+\Delta t\left\|\mathbf{U}^{k}-\mathbf{P} \mathbf{u}_{h}^{k}\right\|_{\mathcal{L}^{2}(\mathcal{O})}\right) \\
& +C \int_{t}^{t_{k+1}}\left\|\widehat{\boldsymbol{\psi}}(s)-\overline{\boldsymbol{\psi}}_{\boldsymbol{h}}^{\boldsymbol{k}}(s)\right\|_{\mathcal{L}^{2}(\mathcal{O})} \mathrm{d} s+C \sqrt{\Delta t}\left\|\boldsymbol{\delta}_{k}\right\|_{\mathcal{L}^{2}\left(\mathcal{O} \times\left(t_{k}, t_{k+1}\right)\right)} .
\end{aligned}
$$


Therefore, by Gronwall's lemma, we get that

$$
\begin{aligned}
\left\|\widehat{\boldsymbol{\psi}}(t)-\overline{\boldsymbol{\psi}}_{\boldsymbol{h}}^{\boldsymbol{k}}(t)\right\|_{\mathcal{L}^{2}(\mathcal{O})} \leqslant C\left(\Delta t\left\|\mathbf{u}_{h}^{k}-\mathbf{U}^{k}\right\|_{\mathcal{L}^{2}(\mathcal{O})}+\left|\boldsymbol{\varepsilon}_{k}\right|+\Delta t\left\|\mathbf{U}^{k}-\mathbf{P} \mathbf{u}_{h}^{k}\right\|_{\mathcal{L}^{2}(\mathcal{O})}\right. \\
\left.+\sqrt{\Delta t}\left\|\boldsymbol{\delta}_{k}\right\|_{\mathcal{L}^{2}\left(\mathcal{O} \times\left(t_{k}, t_{k+1}\right)\right)}\right) .
\end{aligned}
$$

In particular for $t=t_{k}$, we obtain that

$$
\begin{aligned}
\left\|\widehat{\boldsymbol{X}}-\overline{\boldsymbol{X}}_{\boldsymbol{h}}^{\boldsymbol{k}}\right\|_{\mathcal{L}^{2}(\mathcal{O})} \leqslant C\left(\Delta t\left\|\mathbf{u}_{h}^{k}-\mathbf{U}^{k}\right\|_{\mathcal{L}^{2}(\mathcal{O})}+\left|\varepsilon_{k}\right|+\Delta t\left\|\mathbf{U}^{k}-\mathbf{P} \mathbf{u}_{h}^{k}\right\|_{\mathcal{L}^{2}(\mathcal{O})}\right. \\
\left.+\sqrt{\Delta t}\left\|\boldsymbol{\delta}_{k}\right\|_{\mathcal{L}^{2}\left(\mathcal{O} \times\left(t_{k}, t_{k+1}\right)\right)}\right)
\end{aligned}
$$

Since $\mathbf{P}$ is an orthogonal projection in $\mathcal{L}^{2}(\mathcal{O})$, we have that

$$
\left\|\mathbf{U}^{k}-\mathbf{P} \mathbf{u}_{h}^{k}\right\|_{\mathcal{L}^{2}(\mathcal{O})} \leqslant\left\|\mathbf{U}^{k}-\mathbf{u}_{h}^{k}\right\|_{\mathcal{L}^{2}(\mathcal{O})}+\left\|\mathbf{P} \mathbf{U}^{k}-\mathbf{U}^{k}\right\|_{\mathcal{L}^{2}(\mathcal{O})} .
$$

Now, since $\mathbf{U}^{k} \in \mathcal{H}_{0}^{1}(\mathcal{O})$ and $\operatorname{div}\left(\mathbf{U}^{k}\right)=0$, there exists a stream function $\psi \in H^{2}(\mathcal{O}) \cap$ $H_{0}^{1}(\mathcal{O})$ of $\mathbf{U}^{k}$, i.e. $\mathbf{U}^{k}=\operatorname{rot} \psi$. Let $\psi_{h}$ be the Lagrange interpolated function of $\psi$ on the triangulation $\mathcal{T}_{h}$. We denote $\widetilde{\mathbf{U}_{h}^{k}}=\operatorname{rot} \psi_{h}$. Since $\widetilde{\mathbf{U}_{h}^{k}} \in \mathcal{R}_{h}$, we have that

$$
\begin{aligned}
\left\|\mathbf{P} \mathbf{U}^{k}-\mathbf{U}^{k}\right\|_{\mathcal{L}^{2}(\mathcal{O})} & \leqslant\left\|\widetilde{\mathbf{U}_{h}^{k}}-\mathbf{U}^{k}\right\|_{\mathcal{L}^{2}(\mathcal{O})}=\left\|\operatorname{rot}\left(\psi-\psi_{h}\right)\right\|_{\mathcal{L}^{2}(\mathcal{O})} \\
& \leqslant C h\|\psi\|_{H^{2}(\mathcal{O})} \leqslant C h\left\|\mathbf{U}^{k}\right\|_{\mathcal{H}^{1}(\mathcal{O})} .
\end{aligned}
$$

The above equation and (6.49), (6.50) imply the result.

\section{$7 \quad$ Proof of the main result.}

We can now prove Theorem 3.2.

First Step. Assume that $h \leq C(\Delta t)^{2}$. We first show that if (3.10) holds and if

$$
\operatorname{dist}\left(B\left(\boldsymbol{\zeta}_{h}^{k}\right), \partial \mathcal{O}\right)>2 \eta, \quad \operatorname{dist}\left(B\left(\boldsymbol{\zeta}_{h}^{k+1}\right), \partial \mathcal{O}\right)>2 \eta,
$$

then there exist two positive constants $C_{0}$ and $C_{1}$ independent of $\Delta t$ and $h$ such that the error $e_{h}^{k}=\left\|\mathbf{U}^{k}-\mathbf{u}_{h}^{k}\right\|_{\mathcal{L}^{2}(\mathcal{O})}+\left|\boldsymbol{\zeta}\left(t_{k}\right)-\boldsymbol{\zeta}_{h}^{k}\right|$ satisfies the following inequality

$$
e_{h}^{k+1} \leqslant e_{h}^{k}\left(1+C_{0} \Delta t\right)+C_{0} \Delta t \beta_{h}^{k}
$$

where

$$
\sum_{k=0}^{N} \beta_{h}^{k} \leqslant C_{1} .
$$

Let us remark that assumption (7.1) together with (3.10) allows us to perform the change of variables defined in Section 5 and to define $\mathbf{U}^{k}, \mathbf{U}^{k+1}$ and $P^{k+1}$ (see (6.16)).

By using (4.50), there exists $\left(\mathbf{U}_{h}^{k+1}, P_{h}^{k+1}\right) \in \mathcal{K}_{h}\left(\boldsymbol{\zeta}_{h}^{k+1}\right) \times M_{h}\left(\boldsymbol{\zeta}_{h}^{k+1}\right)$ such that

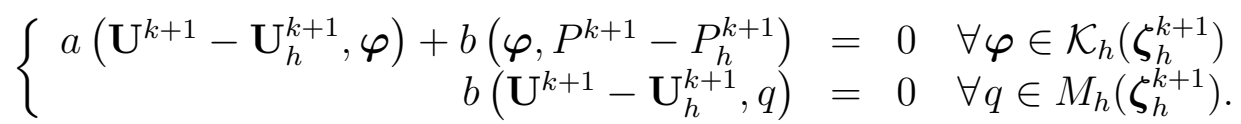


Substracting (7.3) and (3.5) from (6.22) yields

$$
\begin{aligned}
\frac{1}{\Delta t}\left(\mathbf{U}^{k+1}-\mathbf{u}_{h}^{k+1}, \boldsymbol{\varphi}\right)+ & a\left(\mathbf{U}_{h}^{k+1}-\mathbf{u}_{h}^{k+1}, \boldsymbol{\varphi}\right)+b\left(\boldsymbol{\varphi}, P_{h}^{k+1}-p_{h}^{k+1}\right) \\
& =\frac{1}{\Delta t}\left(\widehat{\mathbf{J}}\left(\mathbf{U}^{k} \circ \widehat{\boldsymbol{X}}\right)-\mathbf{u}_{h}^{k} \circ \overline{\boldsymbol{X}}_{\boldsymbol{h}}^{k}, \boldsymbol{\varphi}\right)+\left(\mathbf{A}_{k}, \boldsymbol{\varphi}\right) \quad \forall \boldsymbol{\varphi} \in \mathcal{K}\left(\boldsymbol{\zeta}_{h}^{k+1}\right), \\
& b\left(\mathbf{U}_{h}^{k+1}-\mathbf{u}_{h}^{k+1}, q\right)=0 \quad \forall q \in M_{h}\left(\boldsymbol{\zeta}_{h}^{k+1}\right) .
\end{aligned}
$$

In particular, for $\boldsymbol{\varphi}=\mathbf{U}_{h}^{k+1}-\mathbf{u}_{h}^{k+1}$ and $q=P_{h}^{k+1}-p_{h}^{k+1}$, we easily obtain that

$$
\begin{aligned}
\left\|\mathbf{U}_{h}^{k+1}-\mathbf{u}_{h}^{k+1}\right\|_{\mathcal{L}^{2}(\mathcal{O})} \leqslant & \left\|\widehat{\mathbf{J}}\left(\mathbf{U}^{k} \circ \widehat{\boldsymbol{X}}\right)-\mathbf{u}_{h}^{k} \circ \overline{\boldsymbol{X}}_{\boldsymbol{h}}^{k}\right\|_{\mathcal{L}^{2}(\mathcal{O})} \\
& +\Delta t\left\|\mathbf{A}_{k}\right\|_{\mathcal{L}^{2}(\mathcal{O})}+\left\|\mathbf{U}^{k+1}-\mathbf{U}_{h}^{k+1}\right\|_{\mathcal{L}^{2}(\mathcal{O})}
\end{aligned}
$$

On the other hand, since

$$
\widehat{\mathbf{J}}=\left(\mathbf{J}_{\mathbf{Y}^{k+1}} \circ \mathbf{X}^{k+1}\right)\left(\mathbf{J}_{\mathbf{X}^{k}} \circ \widehat{\boldsymbol{X}}\right)
$$

we have that

$$
\begin{aligned}
\left\|\widehat{\mathbf{J}}\left(\mathbf{U}^{k} \circ \widehat{\boldsymbol{X}}\right)-\mathbf{u}_{h}^{k} \circ \overline{\boldsymbol{X}}_{\boldsymbol{h}}^{\boldsymbol{k}}\right\|_{\mathcal{L}^{2}(\mathcal{O})} \leqslant & C\left\|\left(\mathbf{J}_{\mathbf{Y}^{k+1}} \circ \mathbf{X}^{k+1}\right)\left(\mathbf{J}_{\mathbf{X}^{k}} \circ \widehat{\boldsymbol{X}}\right)-\mathbf{I d}\right\|_{\mathcal{L}^{2}(\mathcal{O})} \\
& +\left\|\mathbf{U}^{k} \circ \widehat{\boldsymbol{X}}-\mathbf{U}^{k} \circ \overline{\boldsymbol{X}}_{\boldsymbol{h}}^{\boldsymbol{k}}\right\|_{\mathcal{L}^{2}(\mathcal{O})} \\
& +\left\|\mathbf{U}^{k} \circ \overline{\boldsymbol{X}}_{\boldsymbol{h}}^{\boldsymbol{k}}-\mathbf{u}_{h}^{k} \circ \overline{\boldsymbol{X}}_{\boldsymbol{h}}^{\boldsymbol{k}}\right\|_{\mathcal{L}^{2}(\mathcal{O})}
\end{aligned}
$$

Since $\left(\mathbf{J}_{\mathbf{Y}^{k+1}} \circ \mathbf{X}^{k+1}\right) \mathbf{J}_{\mathbf{X}^{k+1}}=\mathbf{I d}$, we infer from Lemma 5.6 that

$$
\begin{aligned}
\left\|\left(\mathbf{J}_{\mathbf{Y}^{k+1}} \circ \mathbf{X}^{k+1}\right)\left(\mathbf{J}_{\mathbf{X}^{k}} \circ \widehat{\boldsymbol{X}}\right)-\mathbf{I d}\right\|_{\mathcal{L}^{2}(\mathcal{O})} \leqslant & C\|\widehat{\boldsymbol{X}}-\mathbf{I d}\|_{\mathcal{L}^{2}(\mathcal{O})}\left|\boldsymbol{\zeta}\left(t_{k}\right)-\boldsymbol{\zeta}_{h}^{k}\right| \\
& +C\left\|\mathbf{J}_{\mathbf{X}^{k}}-\mathbf{J}_{\mathbf{X}^{k+1}}\right\|_{\mathcal{L}^{2}(\mathcal{O})} .
\end{aligned}
$$

By using Lemmae 6.4, 6.5 and the above inequality, we obtain that

$$
\begin{aligned}
\|\left(\mathbf{J}_{\mathbf{Y}^{k+1}} \circ \mathbf{X}^{k+1}\right)\left(\mathbf{J}_{\mathbf{X}^{k}} \circ \widehat{\boldsymbol{X}}\right) & -\mathbf{I d} \|_{\mathcal{L}^{2}(\mathcal{O})} \leqslant C\left(\Delta t\left|\boldsymbol{\zeta}\left(t_{k}\right)-\boldsymbol{\zeta}_{h}^{k}\right|\right. \\
& \left.+\Delta t\left\|\mathbf{u}_{h}^{k}-\mathbf{U}^{k}\right\|_{\mathcal{L}^{2}(\mathcal{O})}+\sqrt{\Delta t}\left\|\boldsymbol{\delta}_{k}\right\|_{\mathcal{L}^{2}\left(\mathcal{O} \times\left(t_{k}, t_{k+1}\right)\right)}+\left|\boldsymbol{\varepsilon}_{k}\right|\right) .
\end{aligned}
$$

By using (3.8) and Lemma 5.6, we easily check that

$$
\left\|\mathbf{U}^{k} \circ \widehat{\boldsymbol{X}}-\mathbf{U}^{k} \circ \overline{\boldsymbol{X}}_{\boldsymbol{h}}^{\boldsymbol{k}}\right\|_{\mathcal{L}^{2}(\mathcal{O})} \leqslant C\left\|\widehat{\boldsymbol{X}}-\overline{\boldsymbol{X}}_{\boldsymbol{h}}^{\boldsymbol{k}}\right\|_{\mathcal{L}^{2}(\mathcal{O})} .
$$

The above inequality, relations (7.4), (7.5), (7.6), Lemma 6.5 and the fact that $\operatorname{det} \mathbf{J}_{\overline{\mathbf{X}}_{h}^{k}}=1$ imply that

$$
\begin{aligned}
\left\|\mathbf{U}_{h}^{k+1}-\mathbf{u}_{h}^{k+1}\right\|_{\mathcal{L}^{2}(\mathcal{O})} \leqslant & C\left(\Delta t\left|\boldsymbol{\zeta}\left(t_{k}\right)-\boldsymbol{\zeta}_{h}^{k}\right|+\Delta t\left\|\mathbf{u}_{h}^{k}-\mathbf{U}^{k}\right\|_{\mathcal{L}^{2}(\mathcal{O})}\right. \\
& \left.+\sqrt{\Delta t}\left\|\boldsymbol{\delta}_{k}\right\|_{\mathcal{L}^{2}\left(\mathcal{O} \times\left(t_{k}, t_{k+1}\right)\right)}+\left|\varepsilon_{k}\right|+h \Delta t\right) \\
& +\left\|\mathbf{U}^{k}-\mathbf{u}_{h}^{k}\right\|_{\mathcal{L}^{2}(\mathcal{O})}+\Delta t\left\|\mathbf{A}_{k}\right\|_{\mathcal{L}^{2}(\mathcal{O})}+\left\|\mathbf{U}^{k+1}-\mathbf{U}_{h}^{k+1}\right\|_{\mathcal{L}^{2}(\mathcal{O})}
\end{aligned}
$$


By using Lemma 4.4, Proposition 6.2 and Lemma 6.1, we have the following inequalities

$$
\begin{gathered}
\left\|\mathbf{U}^{k+1}-\mathbf{U}_{h}^{k+1}\right\|_{\mathcal{L}^{2}(\mathcal{O})} \leqslant C h \\
\left\|\mathbf{A}_{k}\right\|_{\mathcal{L}^{2}(\mathcal{O})} \leqslant C\left(\left|\boldsymbol{\zeta}\left(t_{k+1}\right)-\boldsymbol{\zeta}_{h}^{k+1}\right|+h+\Delta t+C \sqrt{\Delta t}\left\|\frac{d^{2}}{d t^{2}}[\mathbf{u} \circ \widetilde{\boldsymbol{\psi}}]\right\|_{\mathcal{L}^{2}\left(\mathcal{O} \times\left(t_{k}, t_{k+1}\right)\right)}\right), \\
\left\|\boldsymbol{\delta}_{k}\right\|_{\mathcal{L}^{2}\left(\mathcal{O} \times\left(t_{k}, t_{k+1}\right)\right)} \leqslant C \Delta t\left\|\frac{\partial \mathbf{u}}{\partial t}\right\|_{\mathcal{L}^{2}\left(\mathcal{O} \times\left(t_{k}, t_{k+1}\right)\right)}, \\
\left|\varepsilon_{k}\right| \leqslant C(\Delta t)^{2} .
\end{gathered}
$$

The above inequalities and (7.7) yield that

$$
\begin{aligned}
&\left\|\mathbf{U}^{k+1}-\mathbf{u}_{h}^{k+1}\right\|_{\mathcal{L}^{2}(\mathcal{O})} \leqslant\left\|\mathbf{U}^{k}-\mathbf{u}_{h}^{k}\right\|_{\mathcal{L}^{2}(\mathcal{O})}+C\left((\Delta t)^{2}+h \Delta t+h\right. \\
&+\Delta t\left|\boldsymbol{\zeta}\left(t_{k+1}\right)-\boldsymbol{\zeta}_{h}^{k+1}\right|+\Delta t\left\|\mathbf{U}^{k}-\mathbf{u}_{h}^{k}\right\|_{\mathcal{L}^{2}(\mathcal{O})} \\
&\left.+(\Delta t)^{3 / 2}\left\|\frac{\partial \mathbf{u}}{\partial t}\right\|_{\mathcal{L}^{2}\left(\mathcal{O} \times\left(t_{k}, t_{k+1}\right)\right)}+(\Delta t)^{3 / 2}\left\|\frac{d^{2}}{d t^{2}}[\mathbf{u} \circ \widetilde{\boldsymbol{\psi}}]\right\|_{\mathcal{L}^{2}\left(\mathcal{O} \times\left(t_{k}, t_{k+1}\right)\right)}\right) .
\end{aligned}
$$

On the other hand, (3.2), (6.7), (6.31) and (6.39) imply that

$$
\begin{aligned}
\left|\boldsymbol{\zeta}\left(t_{k+1}\right)-\boldsymbol{\zeta}_{h}^{k+1}\right| & \leqslant\left|\boldsymbol{\zeta}\left(t_{k}\right)-\boldsymbol{\zeta}_{h}^{k}\right|+\Delta t\left|\mathbf{u}_{h}^{k}\left(\boldsymbol{\zeta}_{h}^{k}\right)-\mathbf{u}\left(\boldsymbol{\zeta}\left(t_{k}\right), t_{k}\right)\right|+\left|\varepsilon_{k}\right| \\
& \leqslant\left|\boldsymbol{\zeta}\left(t_{k}\right)-\boldsymbol{\zeta}_{h}^{k}\right|+C \Delta t|| \mathbf{u}_{h}^{k}-\mathbf{U}^{k} \|_{\mathcal{L}^{2}(\mathcal{O})}+\left|\varepsilon_{k}\right|
\end{aligned}
$$

Combining (7.8) and (7.9), we obtain that

$$
\begin{aligned}
\left\|\mathbf{U}^{k+1}-\mathbf{u}_{h}^{k+1}\right\|_{\mathcal{L}^{2}(\mathcal{O})} & +\left|\boldsymbol{\zeta}\left(t_{k+1}\right)-\boldsymbol{\zeta}_{h}^{k+1}\right| \\
\leqslant(1+C \Delta t)\left(\left|\boldsymbol{\zeta}\left(t_{k}\right)-\boldsymbol{\zeta}_{h}^{k}\right|\right. & \left.+\left\|\mathbf{u}_{h}^{k}-\mathbf{U}^{k}\right\|_{\mathcal{L}^{2}(\mathcal{O})}\right) \\
+ & C\left(h+(\Delta t)^{2}+h \Delta t+(\Delta t)^{3 / 2}\left\|\frac{\partial \mathbf{u}}{\partial t}\right\|_{\mathcal{L}^{2}\left(\mathcal{O} \times\left(t_{k}, t_{k+1}\right)\right)}\right. \\
& \left.+(\Delta t)^{3 / 2}\left\|\frac{d^{2}}{d t^{2}}[\mathbf{u} \circ \widetilde{\boldsymbol{\psi}}]\right\|_{\mathcal{L}^{2}\left(\mathcal{O} \times\left(t_{k}, t_{k+1}\right)\right)}\right) .
\end{aligned}
$$

The above inequality and the hypothesis $h \leqslant C(\Delta t)^{2}$ imply the existence of a positive constant $C_{0}$ such that

$$
\begin{aligned}
\left\|\mathbf{U}^{k+1}-\mathbf{u}_{h}^{k+1}\right\|_{\mathcal{L}^{2}(\mathcal{O})} & +\left|\boldsymbol{\zeta}\left(t_{k+1}\right)-\boldsymbol{\zeta}_{h}^{k+1}\right| \\
& \leqslant\left(1+C_{0} \Delta t\right)\left(\left|\boldsymbol{\zeta}\left(t_{k}\right)-\boldsymbol{\zeta}_{h}^{k}\right|+\left\|\mathbf{u}_{h}^{k}-\mathbf{U}^{k}\right\|_{\mathcal{L}^{2}(\mathcal{O})}\right) \\
& +C_{0} \Delta t\left(\Delta t+\left\|\frac{\partial \mathbf{u}}{\partial t}\right\|_{\mathcal{L}^{2}\left(\mathcal{O} \times\left(t_{k}, t_{k+1}\right)\right)}^{2}+\left\|\frac{d^{2}}{d t^{2}}[\mathbf{u} \circ \widetilde{\boldsymbol{\psi}}]\right\|_{\mathcal{L}^{2}\left(\mathcal{O} \times\left(t_{k}, t_{k+1}\right)\right)}^{2}\right)
\end{aligned}
$$

which is exactly (7.2).

Second Step. We show that if $\Delta t$ is small enough, then the error $e_{h}^{k}=\left\|\mathbf{U}^{k}-\mathbf{u}_{h}^{k}\right\|_{\mathcal{L}^{2}(\mathcal{O})}+$ $\left|\boldsymbol{\zeta}\left(t_{k}\right)-\boldsymbol{\zeta}_{h}^{k}\right|$ satisfies $e_{h}^{k} \leqslant C_{1} \Delta t$ with a constant $C_{1}$ independent of $k, \Delta t$ and $h$. This fact implies, in particular, that (7.1) holds. 
Define

$$
C_{1}=C_{0} \exp \left(C_{0} T\right)\left(\left\|\frac{\partial \mathbf{u}}{\partial t}\right\|_{\mathcal{L}^{2}(\mathcal{O} \times(0, T))}^{2}+\left\|\frac{d^{2}}{d t^{2}}[\mathbf{u} \circ \widetilde{\psi}]\right\|_{\mathcal{L}^{2}(\mathcal{O} \times(0, T))}^{2}\right)+\exp \left(C_{0} T\right) .
$$

It can be easily checked that

$$
\begin{aligned}
\left(1+C_{0} \Delta t\right)^{n} C_{0}\left(\left\|\frac{\partial \mathbf{u}}{\partial t}\right\|_{\mathcal{L}^{2}(\mathcal{O} \times(0, T))}^{2}+\left\|\frac{d^{2}}{d t^{2}}[\mathbf{u} \circ \widetilde{\boldsymbol{\psi}}]\right\|_{\mathcal{L}^{2}(\mathcal{O} \times(0, T))}^{2}\right) & \\
& +\left(1+C_{0} \Delta t\right)^{n}-1 \leqslant C_{1} \quad \forall n \in\{0, \ldots, N\} .
\end{aligned}
$$

Moreover, there exists a positive constant $C_{2}$ such that

$$
\left\|\mathbf{U}^{k}\right\|_{\mathcal{L}^{2}(\mathcal{O})} \leqslant C_{2}
$$

Let $N_{0} \in \mathbb{N}$ be such that $\left(2 C_{1}+C_{2}\right) \Delta t<\eta$, for all $N \geqslant N_{0}$. We next prove by induction over $k$ that, for $N \geqslant N_{0}$ and for $k \in\{0, \ldots, N\}$ we have

$$
\begin{aligned}
\left|\boldsymbol{\zeta}\left(t_{k}\right)-\boldsymbol{\zeta}_{h}^{k}\right| & +\left\|\mathbf{u}_{h}^{k}-\mathbf{U}^{k}\right\|_{\mathcal{L}^{2}(\mathcal{O})} \leqslant\left[\left(1+C_{0} \Delta t\right)^{k}-1\right. \\
& \left.+C_{0}\left(1+C_{0} \Delta t\right)^{k}\left(\left\|\frac{\partial \mathbf{u}}{\partial t}\right\|_{\mathcal{L}^{2}\left(\mathcal{O} \times\left(0, t_{k}\right)\right)}^{2}+\left\|\frac{d^{2}}{d t^{2}}[\mathbf{u} \circ \widetilde{\boldsymbol{\psi}}]\right\|_{\mathcal{L}^{2}\left(\mathcal{O} \times\left(0, t_{k}\right)\right)}^{2}\right)\right] \Delta t .
\end{aligned}
$$

The relation (7.10) is true for $k=0$. Suppose that we have shown (7.10) for a given $k \geqslant 0$. Then, we deduce that

$$
\left|\zeta\left(t_{k}\right)-\zeta_{h}^{k}\right| \leqslant C_{1} \Delta t<\eta
$$

and therefore, by using (3.10), we have that $\operatorname{dist}\left(B\left(\boldsymbol{\zeta}_{h}^{k}\right), \partial \mathcal{O}\right)>2 \eta$.

By using (3.2) and (3.10), we also have that

$$
\begin{aligned}
\left|\boldsymbol{\zeta}_{h}^{k+1}-\boldsymbol{\zeta}_{h}^{k}\right| & \leqslant \frac{1}{\sqrt{\pi}}\left(\left\|\mathbf{U}^{k}-\mathbf{u}_{h}^{k}\right\|_{\mathcal{L}^{2}(\mathcal{O})}+\left\|\mathbf{U}^{k}\right\|_{\mathcal{L}^{2}(\mathcal{O})}\right) \Delta t \\
& \leqslant \frac{C_{1}+C_{2}}{\sqrt{\pi}} \Delta t
\end{aligned}
$$

The above relation, the fact that $\left(2 C_{1}+C_{2}\right) \Delta t<\eta$ and (7.11) imply that $\operatorname{dist}\left(B\left(\boldsymbol{\zeta}_{h}^{k+1}\right), \partial \mathcal{O}\right)>$ $2 \eta$.

Thus, we can apply the first step of the proof to obtain that

$$
\begin{aligned}
\left|\boldsymbol{\zeta}\left(t_{k+1}\right)-\boldsymbol{\zeta}_{h}^{k+1}\right|+ & \left\|\mathbf{u}_{h}^{k+1}-\mathbf{U}^{k+1}\right\|_{\mathcal{L}^{2}(\mathcal{O})} \\
& \leqslant\left(1+C_{0} \Delta t\right)\left(\left|\boldsymbol{\zeta}\left(t_{k}\right)-\boldsymbol{\zeta}_{h}^{k}\right|+\left\|\mathbf{u}_{h}^{k}-\mathbf{U}^{k}\right\|_{\mathcal{L}^{2}(\mathcal{O})}\right) \\
& +C_{0} \Delta t\left(\Delta t+\left\|\frac{\partial \mathbf{u}}{\partial t}\right\|_{\mathcal{L}^{2}\left(\mathcal{O} \times\left(t_{k}, t_{k+1}\right)\right)}^{2}+\left\|\frac{d^{2}}{d t^{2}}[\mathbf{u} \circ \widetilde{\boldsymbol{\psi}}]\right\|_{\mathcal{L}^{2}\left(\mathcal{O} \times\left(t_{k}, t_{k+1}\right)\right)}^{2}\right) .
\end{aligned}
$$


The above relation and (7.10) imply that

$$
\begin{aligned}
&\left|\boldsymbol{\zeta}\left(t_{k+1}\right)-\boldsymbol{\zeta}_{h}^{k+1}\right|+\left\|\mathbf{u}_{h}^{k+1}-\mathbf{U}^{k+1}\right\|_{\mathcal{L}^{2}(\mathcal{O})} \leqslant\left(1+C_{0} \Delta t\right)\left[\left(1+C_{0} \Delta t\right)^{k}-1\right] \Delta t \\
&+C_{0}\left(1+C_{0} \Delta t\right)^{k+1}\left(\left\|\frac{\partial \mathbf{u}}{\partial t}\right\|_{\mathcal{L}^{2}\left(\mathcal{O} \times\left(0, t_{k}\right)\right)}^{2}+\left\|\frac{d^{2}}{d t^{2}}[\mathbf{u} \circ \widetilde{\boldsymbol{\psi}}]\right\|_{\mathcal{L}^{2}\left(\mathcal{O} \times\left(0, t_{k}\right)\right)}^{2}\right) \Delta t \\
&+C_{0} \Delta t\left(\Delta t+\left\|\frac{\partial \mathbf{u}}{\partial t}\right\|_{\mathcal{L}^{2}\left(\mathcal{O} \times\left(t_{k}, t_{k+1}\right)\right)}^{2}+\left\|\frac{d^{2}}{d t^{2}}[\mathbf{u} \circ \widetilde{\boldsymbol{\psi}}]\right\|_{\mathcal{L}^{2}\left(\mathcal{O} \times\left(t_{k}, t_{k+1}\right)\right)}^{2}\right),
\end{aligned}
$$

which implies (7.10) for $k+1$.

Third Step. From the previous steps we conclude that if $\Delta t$ is small enough and if $h \leqslant C(\Delta t)^{2}$ then

$$
\left|\boldsymbol{\zeta}\left(t_{k}\right)-\boldsymbol{\zeta}_{h}^{k}\right|+\left\|\mathbf{u}_{h}^{k}-\mathbf{U}^{k}\right\|_{\mathcal{L}^{2}(\mathcal{O})} \leqslant C_{1} \Delta t \quad \forall k \in\{0, \ldots, N\} .
$$

The above relation, Lemma 5.6, (3.8) and Lemma 4.4 imply that if $\Delta t$ is small enough and if $h \leqslant C(\Delta t)^{2}$ then

$$
\left|\boldsymbol{\zeta}\left(t_{k}\right)-\boldsymbol{\zeta}_{h}^{k}\right|+\left\|\mathbf{u}_{h}^{k}-\mathbf{u}\left(t_{k}\right)\right\|_{\mathcal{L}^{2}(\mathcal{O})} \leqslant C \Delta t \quad \forall k \in\{0, \ldots, N\},
$$

which is the conclusion of the theorem.

\section{Concluding remarks}

We implemented the numerical method we proposed and several numerical tests have been performed. Let us briefly describe the results obtained in the case of a rigid ball falling vertically under the action of a vertical force oriented downwards. At instant $t=0$ the velocity field in the fluids and in the solid is supposed to vanish.

We use a mesh with 1432 triangles and 752 vertices (see Fig. 2 below).

Far from the ball the space discretization parameter is $h_{1} \approx 0.57$ whereas in the neighborhood of the ball it is given by $h_{2} \approx 0.12$ we choose the time step $\Delta t=0.1$ Moreover, we choose the radius of the ball equal to 0.3 , the viscosity $\mu=1$ and the downwards force of intensity equal to one (all quantities are given in International System (IS) units). In Figure 3 we represented the configuration of the system for $k=460$ (corresponding to $t=46.0)$.

We repeated the calculation twice by dividing each the mesh size by two (this means that each triangle was each time divided in four smaller triangles). More precisely, we used the meshes described in the table below.

\begin{tabular}{|c|c|c|c|c|}
\hline & $h$ & Triangles & Vertices & CPU Time \\
\hline \hline Mesh 1 & 0.12 & 1432 & 752 & 3 hours \\
\hline Mesh 2 & 0.06 & 5728 & 2935 & 11 hours \\
\hline Mesh 3 & 0.03 & 22912 & 11597 & 8 days \\
\hline
\end{tabular}

The last column represents the time used by a Pentium IV computer with a $2.4 \mathrm{GHz} \mathrm{CPU}$ clock to achieve the calculation.

In Figure 4 we represented the height of the center of the ball versus the time $t$ for the different meshes. 


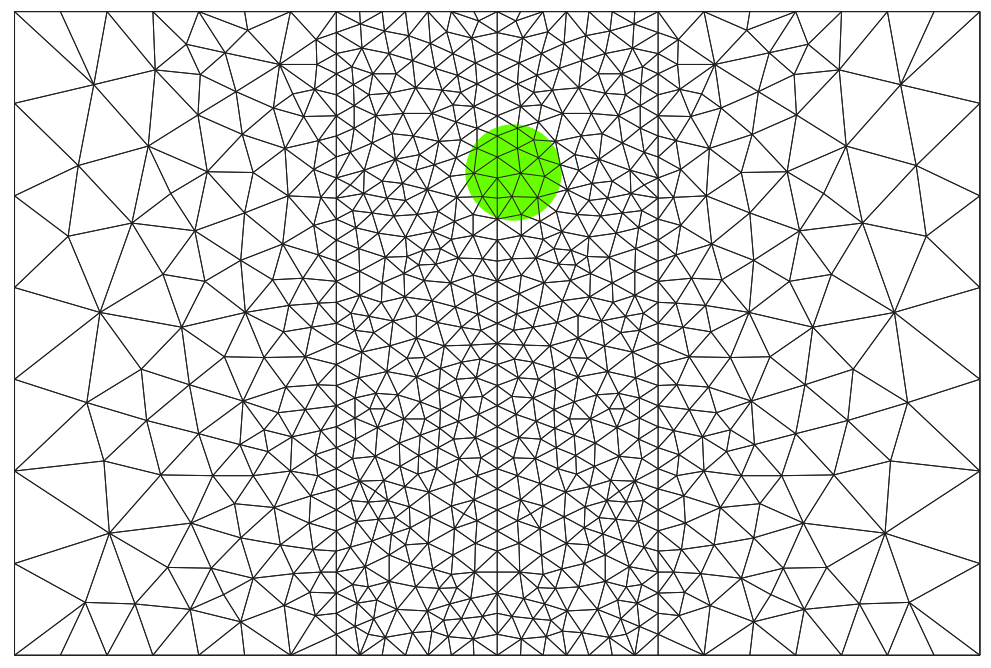

Figure 2: Initial position and mesh

$\mathrm{k}=460, \quad \mathrm{t}=46.0, \quad \mathrm{Vmax}=0.021087$

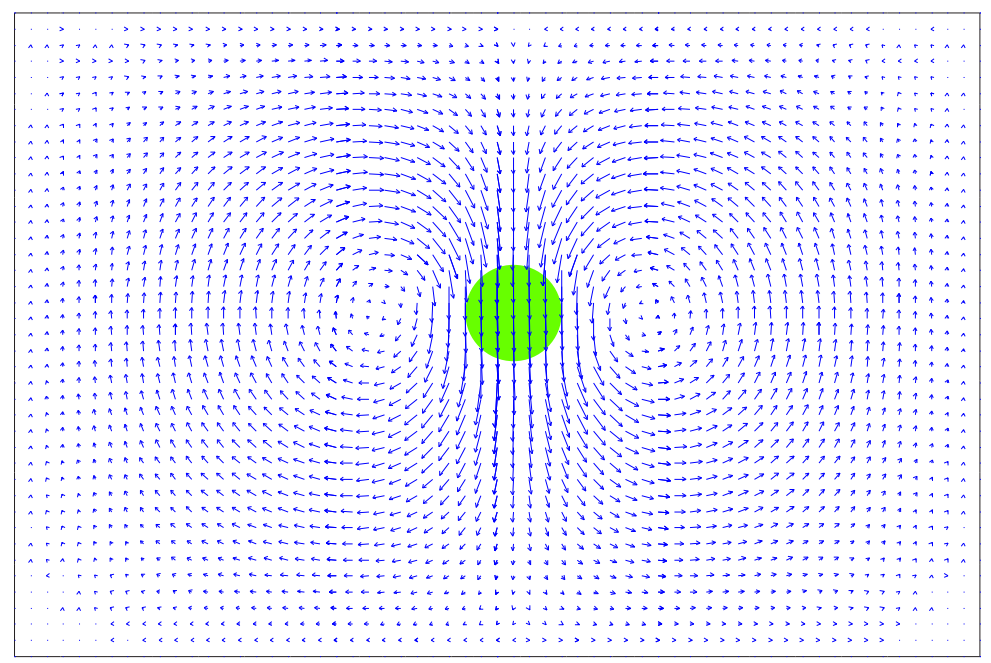

Figure 3: Position and velocity field at time $t=46.0$ 


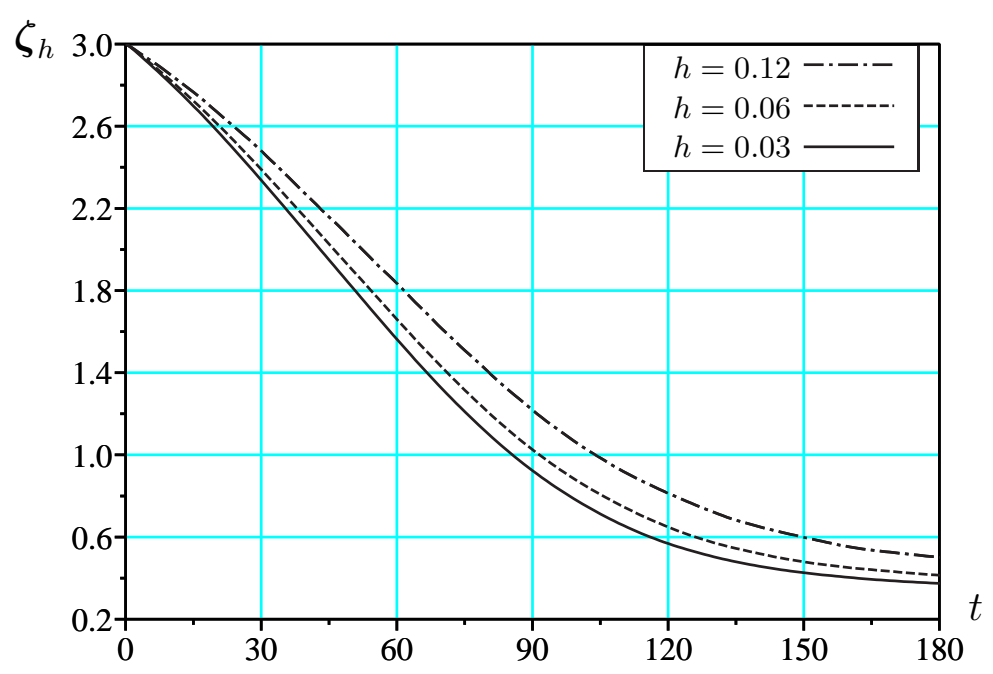

Figure 4: Position of the ball

\section{Acknowledgements}

The first author was partially supported by Conicyt under grant Fondecyt 1010402 and by the Center for Mathematical Modelling from Chile. The fourth author was partially supported by Conicyt under grant Fondecyt 7010402.

\section{References}

[1] V. Arnold, Ordinary differential equations, Springer-Verlag, Berlin, 1992. Translated from the third Russian edition by Roger Cooke.

[2] S. Brenner And L. Scott, The mathematical theory of finite element methods, vol. 15 of Texts in Applied Mathematics, Springer-Verlag, New York, 1994.

[3] H. Brezis, Analyse fonctionnelle, Collection Mathématiques Appliquées pour la Maîtrise. [Collection of Applied Mathematics for the Master's Degree], Masson, Paris, 1983. Théorie et applications. [Theory and applications].

[4] P.-G. Ciarlet, The finite element method for elliptic problems, North-Holland Publishing Co., Amsterdam, 1978. Studies in Mathematics and its Applications, Vol. 4.

[5] C. Conca, H. San Martín, and M. Tucsnak, Existence of solutions for the equations modelling the motion of a rigid body in a viscous fluid, Comm. Partial Differential Equations, 25 (2000), pp. 1019-1042. 
[6] B. Desjardins and M. Esteban, On weak solutions for fluid-rigid structure interaction: compressible and incompressible models, Comm. Partial Differential Equations, 25 (2000), pp. 1399-1413.

[7] L. Eisenhart, Riemannian Geometry, Princeton University Press, Princeton, N. J., 1949. 2d printing.

[8] C. Farhat, P. Geuzaine, and C. Grandmont, The discrete geometric conservation law and the nonlinear stability of ALE schemes for the solution of flow problems on moving grids, J. Comput. Phys., 174 (2001), pp. 669-694.

[9] L. Formaggia And F. Nobile, A stability analysis for the arbitrary Lagrangian Eulerian formulation with finite elements, East-West J. Numer. Math., 7 (1999), pp. 105-131.

[10] H. Fujita And N. Sauer, On existence of weak solutions of the Navier-Stokes equations in regions with moving boundaries, J. Fac. Sci. Univ. Tokyo Sect. I, 17 (1970), pp. 403-420.

[11] L. Gastaldi, A priori error estimates for the arbitrary Lagrangian Eulerian formulation with finite elements, East-West J. Numer. Math., 9 (2001), pp. 123-156.

[12] V. Girault And P.-A. Raviart, Finite element approximation of the NavierStokes equations, Springer-Verlag, Berlin, 1979.

[13] R. Glowinski, T.-W. Pan, T. Hesla, D. Joseph, and J. Périaux, A distributed Lagrange multiplier/fictitious domain method for the simulation of flow around moving rigid bodies: application to particulate flow, Comput. Methods Appl. Mech. Engrg., 184 (2000), pp. 241-267. Vistas in domain decomposition and parallel processing in computational mechanics.

[14] R. Glowinski, T.-W. Pan, T. I. Hesla, D. D. Joseph, and J. Périaux, A fictitious domain approach to the direct numerical simulation of incompressible viscous flow past moving rigid bodies: application to particulate flow, J. Comput. Phys., 169 (2001), pp. 363-426.

[15] C. Grandmont, V. Guimet, And Y. Maday, Numerical analysis of some decoupling techniques for the approximation of the unsteady fluid structure interaction, Math. Models Methods Appl. Sci., 11 (2001), pp. 1349-1377.

[16] C. Grandmont And Y. Maday, Existence for an unsteady fluid-structure interaction problem, M2AN Math. Model. Numer. Anal., 34 (2000), pp. 609-636.

[17] M. Gunzburger, H.-C. Lee, And G. Seregin, Global existence of weak solutions for viscous incompressible flows around a moving rigid body in three dimensions, J. Math. Fluid Mech., 2 (2000), pp. 219-266.

[18] A. Inoue And M. Wakimoto, On existence of solutions of the Navier-Stokes equation in a time dependent domain, J. Fac. Sci. Univ. Tokyo Sect. IA Math., 24 (1977), pp. 303-319. 
[19] B. Maury, A many-body lubrication model, C. R. Acad. Sci. Paris Sér. I Math., 325 (1997), pp. 1053-1058.

[20] — Direct simulations of 2 D fluid-particle flows in biperiodic domains, J. Comput. Phys., 156 (1999), pp. 325-351.

[21] B. Maury And R. Glowinski, Fluid-particle flow: a symmetric formulation, C. R. Acad. Sci. Paris Sér. I Math., 324 (1997), pp. 1079-1084.

[22] F. NobiLe, Numerical approximation of fluid-structure interaction problems with application to haemodynamics, Thèse de doctorat de l'École Polytechnique Fédérale de Lausanne, (2001).

[23] O. Pironneau, On the transport-diffusion algorithm and its applications to the Navier-Stokes equations, Numer. Math., 38 (1982), pp. 309-332.

[24] A. Quarteroni And A. VAlli, Numerical approximation of partial differential equations, vol. 23 of Springer Series in Computational Mathematics, Springer-Verlag, Berlin, 1994.

[25] J. San Martín, V. Starovoitov, and M. Tucsnak, Global weak solutions for the two-dimensional motion of several rigid bodies in an incompressible viscous fluid, Arch. Ration. Mech. Anal., 161 (2002), pp. 113-147.

[26] E. SüLI, Convergence and nonlinear stability of the Lagrange-Galerkin method for the Navier-Stokes equations, Numer. Math., 53 (1988), pp. 459-483.

[27] T. TAKAHASHI, Existence of strong solutions for the equations modelling the motion of a rigid-fluid system in a bounded domain, Advances in differential equations. to appear.

[28] —_, Analyse des équations modélisant le mouvement des systèmes couplant des solides rigides et des fluides visqueux, Thèse de doctorat de l'Université Henri Poincaré - Nancy I, (2002).

[29] R. Temam, Problèmes mathématiques en plasticité, Gauthier-Villars, Montrouge, 1983. 